\title{
4
}

\section{Microscopic Theory of Transport Phenomenon in Hamiltonian Chaotic Systems}

\author{
Shiwei Yan \\ College of Nuclear Science and Technology, Beijing Normal University \\ China
}

\section{Introduction}

It has been one of the important fields of contemporary science to explore the microscopic origin of the damping phenomenon of collective motion in the finite many-body system. The evolution of the early universe, that of the chemical reaction, many active processes in biological system, the fission and fusion processes in nuclear system, the quantum correspondent of the classically chaotic system and the measurement theory are typical examples among others(1-14). Although these processes have been successfully described by the phenomenological transport equation, there still remain some basic problems, such as, how to derive the macro-level transport equation describing the macroscopic irreversible motion from the micro-level reversible dynamics from the fundamental level dynamics; how the statistical state is realized in the irrelevant subsystem and why the irreversible macro-level process is generated as a result of the reversible micro-level dynamics.

The study on this subject has been one of the most fundamental and challenging fields in the various fields of contemporary science. Intensive studies have been carried out (15-29), however, an acceptance microscopic understanding is still far from realization and there still remain a lot of studies(30-33).

\subsection{From reversible to irreversible: Foundation of statistical physics}

Before discussing the microscopic origin of irreversibility phenomenon, one should be aware of an informal indication of the problem in explaining the meaning of the foundation of statistical physics, which can be seen in the presence of two types of processes: (1) time-irreversible macroscopic (relevant or collective) process which obey the thermodynamics, or kinetic, laws; and (2) time-reversible microscopic (irrelevant or intrinsic) process which obey, say, the Newton and Maxwell equations of motion. The great difference between both descriptions of the processes in nature, is not clearly understood and an acceptance explanation on the origin of irreversibility is still lack(34). The formal definition of the problem is to derive a macroscopic equation which describes an irreversible evolution which begins with a reversible Hamiltonian equation.

Before the recognition of the importance of chaos, the attempts to unite, in a formal way, the statistical and dynamical description of a system, usually starts with dividing the total system into the relevant and irrelevant degrees of freedom by hand and assuming the irrelevant system placed in a state of micro-canonical equilibrium (thermodynamical) state 
(Ref. Boltzmann's pioneer proposal(35) and the textbooks as (36)). This is the Boltzmann principle

$$
S=k \ln \mathcal{A}(E) .
$$

where $\mathcal{A}(E)$ is the area of the phase space explored by the system in a micro-canonical state, $k$ the Boltzmann constant and $S$ the entropy. However, such the derivations are essentially based on the assumption of an infinitely large number of degrees of freedom and the existence of two different time scales $\tau_{r} \gg \tau_{i}$, where $\tau_{r}$ is the time scale of relevant degrees of freedom and $\tau_{i}$ is that of irrelevant ones. Under such assumption, the irreversibility or the equilibrium condition is supplemented for the dynamical equations, and the resultant distribution has the form

$$
P(\epsilon) \sim e^{-\beta \epsilon} \quad \beta \equiv 1 / k T
$$

where $\epsilon$ is the energy of the irrelevant degrees of freedom and T the temperature of the system. Distribution (2) is called the equilibrium Boltzmann distribution which corresponds to the thermodynamical state and thus only can be adopted to describe the equilibrium process rather than nonequilibrium states. It also should be mentioned that Poincaré recurrence theorem says that for a finite and area-preserving motion, any trajectory should return to an arbitrarily taken domain $\Sigma$ in a finite time and should do so repeatedly for an infinite number of time. However, Boltzmann had rightly argue that for a large number of particles, this recurrence time would be astronomically long. This assumption only can be justified for a system with infinite number of degrees of freedom. When one want to study the origin of irreversibility of a finite system where dynamical chaos occurs, this assumption of infinite occurrence time will cause some serious problems.

Many attempts have been successfully achieved in different ways and with some supplementary conditions on microscopic Hamiltonian equations, such as the random phase approximation (mixing)(37; 38), Gaussian orthogonal ensemble (GOE), or other equivalent conditions played the role of statistical element $(15-20 ; 25-29 ; 39)$. The ergodic and irreversible property is assumed for the irrelevant system with infinite number of degree of freedom. Temperature, so as thermodynamics, is introduced by hand through the supplementary conditions. From dynamical point of view, such the derivation is unsatisfactory since the strong condition like randomness of some variables or statistical ansatz should be introduced by hand. Following the recognition of the importance of chaos, it has been supposed that there is an intimate relation between the realization of irreversibility and the order-to-chaos transition within the microscopic Hamilton dynamics. When one derives the macroscopic irreversibility from the fundamental Hamiltonian equations, the stochastic processes can be obtained for some specific parameters and initial conditions. However even in this case, such the supplementary assumptions still remain to be justified. The main problems are: whether or not one may substantiate statistical state in the way dynamical chaos is structured in real Hamiltonian system, whether or not there are some difficulties of using the properties of dynamical chaos as a source of randomness, whether one can derive a random process from the dynamical chaotic motion and whether or in what conditions, the derived stochastic process may correspond to the above-mentioned random assumptions. It is well known that Poincare recurrence time is finite for finite Hamiltonian systems and its phase space has fractal (or multi-fractal) structure, where the ergodicity of motion is not generally satisfied. Therefore, specially in the finite system, it is not a trivial discussion whether or not the irrelevant subsystem can be effectively replaced by a statistical object as a heat bath, even when it shows chaotic behavior 
and its Lyapunov exponent has a positive value everywhere in the phase space. It is also an interesting question to explore the relation between the dynamical definition of the statistical state, if it exists, and the static definition of it.

\subsection{From infinite to finite system}

It should say that there is substantial difficulty, or it is almost impossible in some extent, for us to derive the macroscopic irreversibility from the fundamental Hamiltonian equations for an infinite system because which has an infinite (or extremely large) number of degrees of freedom. This fact is generally accepted as the reason for the introduction of the statistical assumptions. On another hand, when one derive macroscopic equations by averaging over microscopic random variables resulted in a reduced description of an in nite system, the detailed structure of micro phase space will lose its importance. In this sense, for an in $\square$ nite system, such the statistical approach seems to be reasonable and will not cause any serious problems.

However, in such systems as atoms, nuclei and biomolecules whose environment is not infinite, where assumption of a large number of degrees of freedom is not justified, and in a case when one intends to derive the macroscopic irreversibility and phenomenological transport equation from the fundamental level dynamics, it is not obvious whether or not one may introduce the statistical assumptions for the irrelevant degrees of freedom. The decisive problem in the finite system is how to justify an introduction of some statistical state or some statistical concept like the temperature or the heat bath, which is one of the basic ingredient to derive an irreversible process to the Hamilton system.

With regards to the damping phenomena observed in the giant resonance on top of the highly excited states in the nuclear system, its microscopic description has been mainly based on the temperature dependent mean-field theory(40-45). However, an introduction of the temperature in the finite-isolated nuclei is by no means obvious, when one explicitly introduce many single-particle excitation modes on top of the temperature-dependent shell model. Could one introduce a chaotic (complex) excitation mode on top of the chaotic (heat bath characterized by the temperature) system? When one considers the $2 p-2 h$ (two-particle two-hole) state as a door way state to be coupled with the $1 p-1 h$ collective excitation mode, a naive classical model Hamilton would be something like a $\beta$ Fermi-Pasta-Ulam (FPU) system(46-49) where no heat bath is assumed, rather than the temperature-dependent shell model where the Matsubara Green function is used.

The recent development in the classical theory of nonlinear Hamiltonian system $(22 ; 34 ; 50$ 53) has shown that there appears an exceedingly rich structure in the phase space, which is usually considered to prevent a fully developed chaos. The existence of fractal structure is a remarkable property of Hamilton chaos and a typical feature of the phase space in real system. Due to the fractal structure of the phase space, the motion of Hamilton system of general type is not ergodic, specially for a finite Hamiltonian system. In this case, the questions then arise: what kind random process corresponds to the dynamical chaotic motion? whether or not the system dynamically reaches some statistical object? The microscopic derivation of the non-equilibrium statistical physics in relation with the exceedingly rich structure of the phase space as well as with the order-to-chaos transition might be explored within the microscopic Hamilton dynamics. This subject will be further discussed in Sec. 4.1.2.

Another important issue related to this study is how to divide the total system into the relevant and irrelevant degrees of freedom. However, in many approaches $(24 ; 26 ; 27)$, one usually starts with dividing the total system into the relevant and irrelevant degrees of freedom 
by hand. In the system where a total number of degrees of freedom can be approximately treated as an infinite, there does not arise any serious problem how to introduce the relevant degrees of freedom. In the finite system as nuclei where a number of the degrees of freedom is not large enough, and a time scale of the relevant motion and that of the irrelevant one is typically less than one order of magnitude difference, there arises an important problem how to distinguish the relevant ones from the rest in a way consistent with the underlying microscopic dynamics for aiming to properly characterize the collective motion. Here it worth to mention another important issue is related with an applicability of the linear response theory (LRT)(54-59), because a validity of the linear approximation for the macro-level dynamics does not necessarily justify that for the micro-level dynamics. Furthermore, there arises a basic question whether or not one may divide the total system into the relevant and irrelevant subsystems by leaving the linear coupling between them(30; 50; 60-62). At the best of our knowledge, it seems that there is no compelling physical reason to choose the linear coupling interaction between the relevant and irrelevant subsystems.

Summarily speaking, in exploring the microscopic dynamics in $\square$ nite Hamiltonian system, says, answering the basic questions as listed in the beginning of this section, there are two main subjects. One is how to divide the total system into the relevant and irrelevant ones and another is how to derive the macroscopic statistical properties from microscopic dynamics.

\subsection{The nonlinear theory of the classical Hamiltonian system}

From above discussion, one can conclude that the theory of chaotic dynamics should play a decisive role in understanding the origin of microscopic irreversibility within the microscopic Hamilton dynamics. It is imperative to remember the recent development in the classical theory of nonlinear Hamilton system $(34 ; 51-53)$.

The chaos phenomenon is often used to describe the motions of the system's trajectories which are sensitive to the slightest changes of the initial condition. The motion known as chaotic occupies a certain area (called stochastic sea) in the phase space. In idea chaos, the stochastic sea is occupied in uniform manner. This is, however, not the case in real systems. The phase space has exceedingly rich structure where the chaotic and regular motions co-exist and there are many islands which a chaotic trajectory can not penetrate (as stated in KAM Theorem). Many important properties of chaotic dynamics, such as the order-to-chaos transition dynamics, are determined by the properties of the motion near the boundary of islands.

Thanks to H. Poincaré and his successors, we know how important it is to understand why there appears an exceedingly rich structure in the phase space and how the order-to-chaos transition occurs, rather than to understand individual trajectory under a specific initial condition. A set of closed orbits having the same property (characterized by a set of local constants of motion) forms a torus structure around a certain stable fixed point in the phase space, and is separated from the other types of closed orbits (characterized by another set of local constants of motion) through separatrices. Depending on the strength of the nonlinear interaction or on the energy of the system, there appear many kinds of new periodic orbits characterized by respective local constants of motion along the known periodic orbits, which are called bifurcation phenomena.

When different separatrices start to overlap in a small region of the phase space, there appears a local chaotic sea. In this overlap region where two kinds of periodic orbits characterized by different sets of constants of motion start to coexist, it might become difficult to realize a well-developed closed orbit characterized by a single set of local constants of motion any 


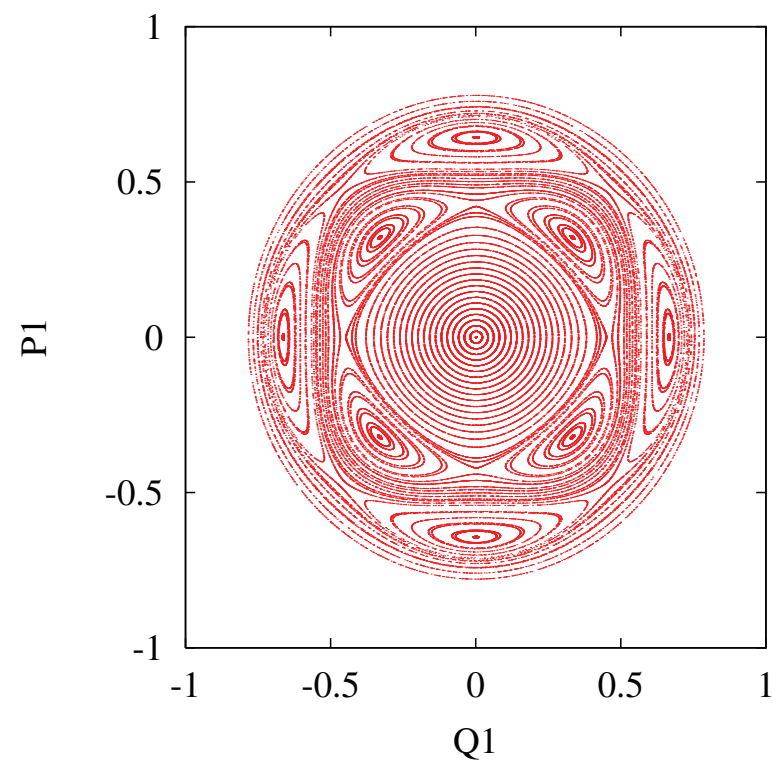

Fig. 1. Poincaré section map of $\mathrm{SU}(3)$ Hamiltonian on $\left(q_{1}, p_{1}\right)$ plane for a case with $E=40$ and $V_{i}=-0.01$

more. This overlap criterion on an appearance of the chaotic sea has been considered to be a microscopic origin of the order-to-chaos transition dynamics.

A classical example of the order-to-chaos transition for the $\mathrm{SU}(3)$ classical Hamiltonian system (66) is shown in Figs. 1 to 4 for the cases with $V_{i}=-0.01,-0.03,-0.045$ and -0.07 , respectively. In the cases with $V_{i}=-0.01$ and -0.03 , i.e., the smaller interaction strength or weaker nonlinearity, the whole phase space is covered by the regular motions, forming many islands structure. When the nonlinearity of interaction goes to stronger, there appears chaotic sea in a region where the crossing point of the separatrics (Fig. 3), i.e., the unstable fixed point is expected in Fig. 2. Moreover, there appear many secondary islands around the primary island structure, which has already existed in Fig. 2. A chaotic trajectory can not penetrate the island and a regular trajectory from an island is not be able to escape from it. Around some secondary island, one may find some tertiary island structure, and so forth, as shown in Fig. 5. This repeated complex structure is called fractal phenomena. The fractal structure of phase space is a remarkable property of Hamiltonian chaos and a typical feature of the phase space in real systems. For a classical Hamiltonian system, the distribution of the Poincare recurrence is time- and space-fractal, which plays a crucial role in an understanding of the general properties of chaotic dynamics and the foundation of statistical physics.

The general theory of the nonlinear dynamics has been developed for aiming to understand these complex structure by studying why there appear many stable and unstable fixed points, and how to get an information on their constants of motion. And also the theory of nonlinear chaotic dynamics plays an important role in understanding the foundation of statistical physics. 


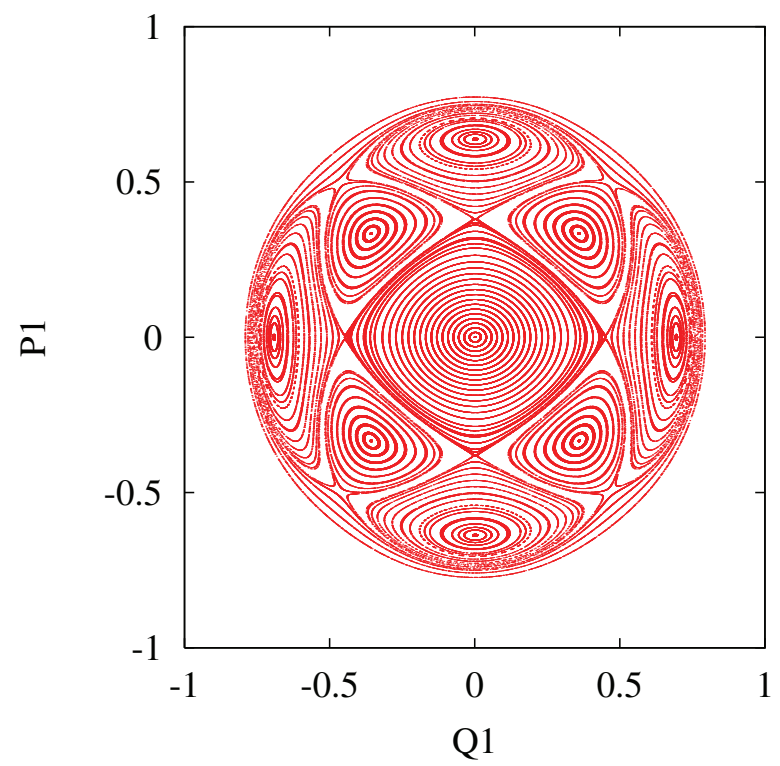

Fig. 2. Poincaré section map of $\mathrm{SU}(3)$ Hamiltonian on $\left(q_{1}, p_{1}\right)$ plane for a case with $E=40$ and $V_{i}=-0.03$

\subsection{The self-consistent collective coordinate (SCC) method \& the optimum coordinate system}

As discussed in Sect. 1.2, for finite system, an important problem is to explore how to divide the total system into the relevant and irrelevant degrees of freedom in a way consistent with the underlying microscopic dynamics for aiming to properly characterize the collective motion. In a case of the Hamiltonian system, a division may be performed by applying the self-consistent collective coordinate (SCC) method(60). In the following, for the sake of self-containedness, the SCC method is briefly reformulated within the classical Hamiltonian system. The detailed description can be found in Ref. (60) and a review article (50).

The SCC method was proposed within the usual symplectic manifold, which intends to define the maximally-decoupled coordinate system where the minimum number of coordinates is required in describing the trajectory under discussion. In such a finite system as the nucleus, it is not obvious how to introduce the relevant (collective or distinguished) coordinates which are used in describing macroscopic properties of the system. This problem is also very important to explore why and how the statistical aspect could appear in the finite nuclear system, and whether or not the irrelevant system could be expressed by some statistical object, because there is no obvious reason to divide the nuclear system into two, unlike a case with the Brownian particle plus molecular system.

The basic idea of the SCC method rests on the following point: The nonlinear canonical transformation between the original coordinate system and a new coordinate one is defined in such a way that the anharmonic effects causing the microscopic structure change of the relevant coordinates are incorporated into the latter coordinate system as much as possible, and the coupling between the relevant and irrelevant subsystems is optimally minimized. 


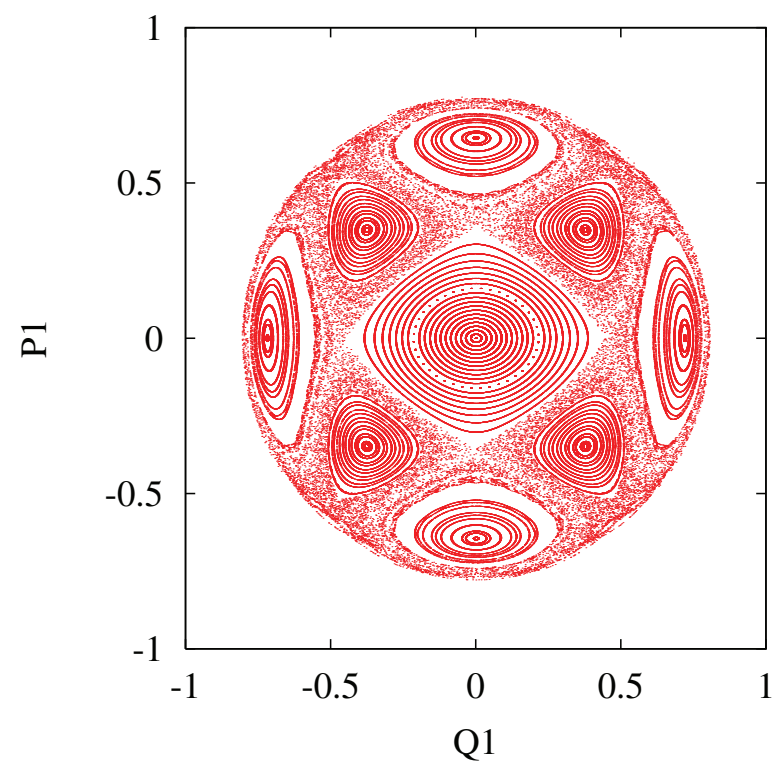

Fig. 3. Poincaré section map of $\mathrm{SU}(3)$ Hamiltonian on $\left(q_{1}, p_{1}\right)$ plane for a case with $E=40$ and $V_{i}=-0.045$

Trajectories in a $2 \mathrm{~K}$ dimensional symplectic manifold expressed by

$$
M^{2 K}: \quad\left\{C_{j}^{*}, C_{j} ; j=1, \cdots, K\right\}
$$

are organized by the canonical equations of motion given as

$$
i \dot{C}_{j}=\frac{\partial H}{\partial C_{j}^{*}}, \quad i \dot{C}_{j}^{*}=-\frac{\partial H}{\partial C_{j}}, \quad j=1, \cdots, K,
$$

where $H$ denotes the Hamiltonian of the system. Let us consider one of the trajectories which are obtained by solving Eq. (4) under a set of specific initial conditions. In describing a given trajectory, it does not matter what coordinate system one may use provided one employs the whole degrees of freedom without any truncation. An arbitrary representation used in describing the canonical equations of motion in Eq. (4) will be called the initial representation (IR). Out of many coordinate systems which are equivalent with each other and are related through the canonical transformations with one another, however, one may select a maximally-decoupled coordinate system where the minimum number of coordinates is required in describing a given trajectory. What one has to do is to extract a small dimensional submanifold denoted as $M^{2 L}(L<K)$ on which the given trajectory is confined. The representation characterizing the maximally-decoupled coordinate system will be called the dynamical representation (DR). Let us introduce a set of canonical variables in the DR. A set of coordinates $\left\{\eta_{a}^{*}, \eta_{a} ; a=1, \cdots, L\right\}$ are called the relevant degrees of freedom and are used in describing the given trajectory, whereas $\left\{\xi_{\alpha}^{*}, \xi_{\alpha} ; \alpha=L+1, \cdots, K\right\}$ are called the irrelevant 


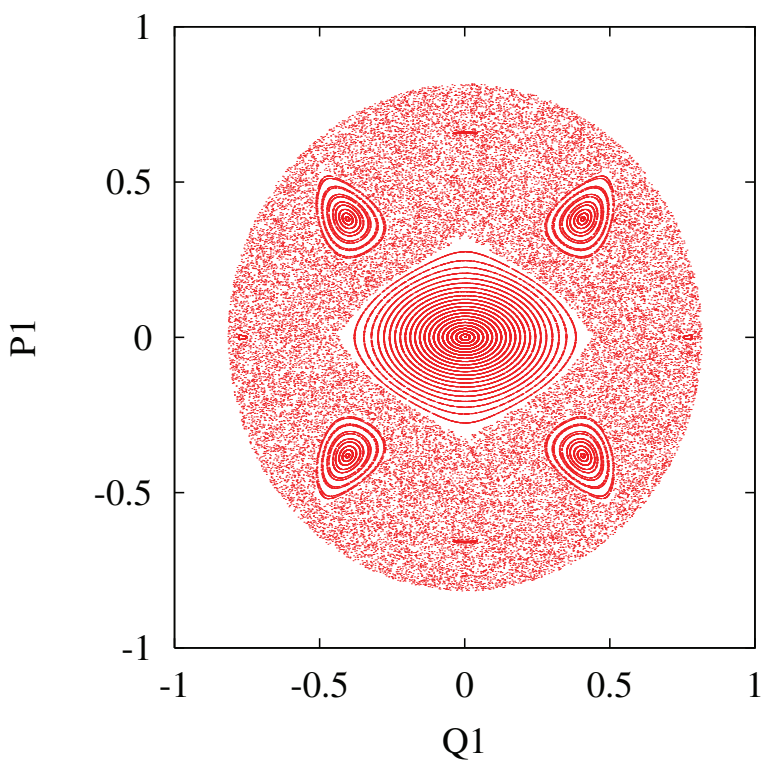

Fig. 4. Poincaré section map of SU(3) Hamiltonian on $\left(q_{1}, p_{1}\right)$ plane for a case with $E=40$ and $V_{i}=-0.07$

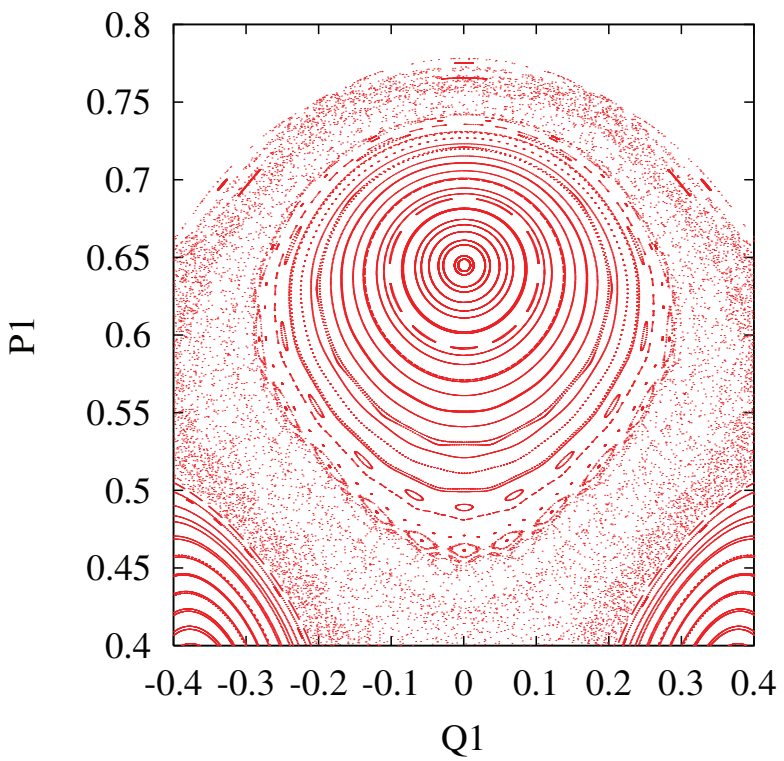

Fig. 5. Magnification of the top island of Fig. 3. 
degrees of freedom, and this new coordinate system provides us with another chart expressed as $I^{2 K}$.

In order to find the DR, one has to know the canonical transformation between IR and DR,

$$
M^{2 K}:\left\{C_{j}^{*}, C_{j}\right\} \Leftrightarrow I^{2 K}: \quad\left\{\eta_{a}^{*}, \eta_{a} ; \xi_{\alpha}^{*}, \xi_{\alpha}\right\}
$$

Ensuring Eq. (5) to be a canonical transformation, there should hold the following canonical variable condition given by

$$
\begin{aligned}
& \sum_{j}\left\{C_{j} \frac{\partial C_{j}^{*}}{\partial \eta_{a}^{*}}-C_{j}^{*} \frac{\partial C_{j}}{\partial \eta_{a}^{*}}\right\}=\eta_{a}+i \frac{\partial S}{\partial \eta_{a}^{*}}, \\
& \sum_{j}\left\{C_{j} \frac{\partial C_{j}^{*}}{\partial \xi_{\alpha}^{*}}-C_{j}^{*} \frac{\partial C_{j}}{\partial \xi_{\alpha}^{*}}\right\}=\xi_{\alpha}+i \frac{\partial S}{\partial \xi_{\alpha}^{*}},
\end{aligned}
$$

where $S$ is a generating function of the canonical transformation, and is an arbitrary real function of $\eta_{a}, \eta_{a}^{*}, \xi_{\alpha}$ and $\xi_{\alpha}^{*}$ satisfying $S^{*}=S$. From Eq. (6), one may obtain the following relations

$$
\begin{aligned}
& \sum_{j}\left\{\frac{\partial C_{j}}{\partial \eta_{b}} \frac{\partial C_{j}^{*}}{\partial \eta_{a}^{*}}-\frac{\partial C_{j}}{\partial \eta_{a}^{*}} \frac{\partial C_{j}^{*}}{\partial \eta_{b}}\right\}=\delta_{a, b}, \\
& \sum_{j}\left\{\frac{\partial C_{j}}{\partial \xi_{\beta}} \frac{\partial C_{j}^{*}}{\partial \xi_{\alpha}^{*}}-\frac{\partial C_{j}}{\partial \xi_{\alpha}^{*}} \frac{\partial C_{j}^{*}}{\partial \xi_{\beta}}\right\}=\delta_{\alpha, \beta}, \\
& \sum_{j}\left\{\frac{\partial C_{j}}{\partial \xi_{\alpha}} \frac{\partial C_{j}^{*}}{\partial \eta_{a}^{*}}-\frac{\partial C_{j}}{\partial \eta_{a}^{*}} \frac{\partial C_{j}^{*}}{\partial \xi_{\alpha}}\right\}=0, \text { etc. }
\end{aligned}
$$

Since the transformation in Eq. (5) is canonical, the new variables in the DR also satisfy the canonical equations of motion given as

$$
i \dot{\eta}_{a}=\frac{\partial H}{\partial \eta_{a}^{*}}, \quad i \dot{\eta}_{a}^{*}=-\frac{\partial H}{\partial \eta_{a}}, \quad i \dot{\xi}_{\alpha}=\frac{\partial H}{\partial \xi_{\alpha}^{*}}, \quad i \dot{\xi}_{\alpha}^{*}=-\frac{\partial H}{\partial \xi_{\alpha}} .
$$

Since the trajectory under consideration is supposed to be described by the relevant degrees of freedom alone, and since the irrelevant degrees of freedom are assumed to describe a small-amplitude motion around it, one may introduce a Taylor expansion of $C_{j}^{*}$ and $C_{j}$ with respect to $\xi_{\alpha}^{*}$ and $\xi_{\alpha}$ on the surface of the submanifold $M^{2 L}$ as

$$
C_{j}=\left[C_{j}\right]+\sum_{\alpha}\left\{\xi_{\alpha}\left[\frac{\partial C_{j}}{\partial \xi_{\alpha}}\right]+\xi_{\alpha}^{*}\left[\frac{\partial C_{j}}{\partial \xi_{\alpha}^{*}}\right]\right\}+\cdots,
$$

where the symbol $[g]$ for an arbitrary function $g\left(\eta_{a}^{*}, \eta_{a} ; \xi_{\alpha}^{*}, \xi_{\alpha}\right)$ denotes a function on the surface $M^{2 L}$, and is a function of the relevant variables alone,

$$
[g] \equiv g\left(\eta_{a}^{*}, \eta_{a} ; \xi_{\alpha}^{*}=0, \xi_{\alpha}=0\right) .
$$


Here, it should be noticed that a set of functions $\left[C_{j}\right]$ and $\left[C_{j}^{*}\right]$ provides us with a knowledge on how the submanifold $M^{2 L}$ is embedded in $M^{2 K}$. In other words, a diffeomorphic mapping

$$
I^{2 L} \rightarrow M^{2 L} \quad \text { embedded in } \quad M^{2 K}: \quad\left\{\eta_{a}^{*}, \eta_{a}\right\} \rightarrow\left\{\left[C_{j}\right],\left[C_{j}^{*}\right]\right\} .
$$

is determined by the set of $\left[C_{j}\right]$ and $\left[C_{j}^{*}\right]$ which are functions of the relevant variables $\eta_{a}^{*}$ and $\eta_{a}$ alone.

In the same way, one may have an expansion form as

$$
\frac{\partial H}{\partial C_{j}^{*}}=\left[\frac{\partial H}{\partial C_{j}^{*}}\right]+\sum_{\alpha}\left\{\xi_{\alpha}\left[\frac{\partial^{2} H}{\partial \xi_{\alpha} \partial C_{j}^{*}}\right]+\xi_{\alpha}^{*}\left[\frac{\partial^{2} H}{\partial \xi_{\alpha}^{*} \partial C_{j}^{*}}\right]\right\}+\cdots, \text { and c.c., }
$$

which appears on the r.h.s. in Eq. (4).

Here, one has to notice that there hold the following relations,

$$
\begin{aligned}
& \frac{\partial H}{\partial \eta_{a}}=\sum_{j}\left\{\frac{\partial C_{j}}{\partial \eta_{a}} \frac{\partial H}{\partial C_{j}}+\frac{\partial C_{j}^{*}}{\partial \eta_{a}} \frac{\partial H}{\partial C_{j}^{*}}\right\}, \\
& \frac{\partial H}{\partial \xi_{\alpha}}=\sum_{j}\left\{\frac{\partial C_{j}}{\partial \xi_{\alpha}} \frac{\partial H}{\partial C_{j}}+\frac{\partial C_{j}^{*}}{\partial \xi_{\alpha}} \frac{\partial H}{\partial C_{j}^{*}}\right\} .
\end{aligned}
$$

Using Eqs. (9) and (12), one may apply the Taylor expansion to the quantities appearing on the lhs in Eq. (13). Its lowest order equation is given as

$$
\begin{aligned}
& {\left[\frac{\partial H}{\partial \eta_{a}}\right]=\sum_{j}\left\{\left[\frac{\partial C_{j}}{\partial \eta_{a}}\right]\left[\frac{\partial H}{\partial C_{j}}\right]+\left[\frac{\partial C_{j}^{*}}{\partial \eta_{a}}\right]\left[\frac{\partial H}{\partial C_{j}^{*}}\right]\right\},} \\
& {\left[\frac{\partial H}{\partial \xi_{\alpha}}\right]=\sum_{j}\left\{\left[\frac{\partial C_{j}}{\partial \xi_{\alpha}}\right]\left[\frac{\partial H}{\partial C_{j}}\right]+\left[\frac{\partial C_{j}^{*}}{\partial \xi_{\alpha}}\right]\left[\frac{\partial H}{\partial C_{j}^{*}}\right]\right\} .}
\end{aligned}
$$

The basic idea of the SCC method formulated within the TDHF theory rests on the invariance principle of the Schrödinger equation(63). In the present case of the classical system, it is expressed as the invariance principle of the canonical equations of motion, which is given by

$$
i \frac{d}{d t}\left[C_{j}\right]=\left[\frac{\partial H}{\partial C_{j}^{*}}\right], \quad i \frac{d}{d t}\left[C_{j}^{*}\right]=-\left[\frac{\partial H}{\partial C_{j}}\right], \quad j=1, \cdots, K .
$$

Since the time-dependence of $\left[C_{j}\right]$ and $\left[C_{j}^{*}\right]$ is supposed to be described by that of $\eta_{a}$ and $\eta_{a}^{*}$, Eq. (15) is expressed as

$$
\left[\frac{\partial H}{\partial C_{j}^{*}}\right]=i \sum_{a}\left\{\dot{\eta}_{a} \frac{\partial\left[C_{j}\right]}{\partial \eta_{a}}+\dot{\eta}_{a}^{*} \frac{\partial\left[C_{j}\right]}{\partial \eta_{a}^{*}}\right\}, \text { and c.c., } j=1, \cdots, K .
$$


Substituting Eq. (16) into the r.h.s. of Eq. (14), one gets

$$
\begin{aligned}
{\left[\frac{\partial H}{\partial \eta_{a}^{*}}\right] } & =i \sum_{b} \dot{\eta}_{b} \sum_{j}\left\{\frac{\partial\left[C_{j}\right]}{\partial \eta_{b}} \frac{\partial\left[C_{j}^{*}\right]}{\partial \eta_{a}^{*}}-\frac{\partial\left[C_{j}\right]}{\partial \eta_{a}^{*}} \frac{\partial\left[C_{j}^{*}\right]}{\partial \eta_{b}}\right\} \\
& +i \sum_{b} \dot{\eta}_{b}^{*} \sum_{j}\left\{\frac{\partial\left[C_{j}\right]}{\partial \eta_{b}^{*}} \frac{\partial\left[C_{j}^{*}\right]}{\partial \eta_{a}^{*}}-\frac{\partial\left[C_{j}\right]}{\partial \eta_{a}^{*}} \frac{\partial\left[C_{j}^{*}\right]}{\partial \eta_{b}^{*}}\right\}, \text { and c.c.. }
\end{aligned}
$$

When there holds the lowest order relation derived from Eq. (6), i.e.,

$$
\text { Condition I } \sum_{j}\left\{\left[C_{j}\right] \frac{\partial\left[C_{j}^{*}\right]}{\partial \eta_{a}^{*}}-\left[C_{j}^{*}\right] \frac{\partial\left[C_{j}\right]}{\partial \eta_{a}^{*}}\right\}=\eta_{a}
$$

called the Canonical Variable Condition, Eq. (17) reduces into

$$
i \dot{\eta}_{a}=\left[\frac{\partial H}{\partial \eta_{a}^{*}}\right], \quad i \dot{\eta}_{a}^{*}=-\left[\frac{\partial H}{\partial \eta_{a}}\right],
$$

which just corresponds to the lowest order equation of Eq. (8), and is called the Equation of Collective Motion.

$$
\text { Condition II } 0=\left[\frac{\partial H}{\partial C_{j}^{*}}\right]-\sum_{a}\left\{\frac{\partial[H]}{\partial \eta_{a}^{*}} \frac{\partial\left[C_{j}\right]}{\partial \eta_{a}}-\frac{\partial[H]}{\partial \eta_{a}} \frac{\partial\left[C_{j}\right]}{\partial \eta_{a}^{*}}\right\}, \text { and c.c., }
$$

which is called the Maximally-Decoupling Condition.

Conditions I and II constitute a set of basic equations of the SCC method in the classical Hamilton system. From the Maximally-Decoupling Condition, one can get the following relation,

$$
\left[\frac{\partial H}{\partial \xi_{\alpha}}\right]=0 .
$$

Equation (21) simply states that the new coordinate system $\left\{\eta_{a}, \eta_{a}^{*}, \xi_{\alpha}, \xi_{\alpha}^{*}\right\}$ determined by the SCC method has no first order couplings between the relevant and irrelevant degrees of freedom. With the aid of the SCC method, one may introduce the maximally-decoupled coordinate system, where the linear coupling between the relevant and irrelevant degrees of freedom is eliminated. This result is very important to treat the collective dissipative motion coupled with the irrelevant system. In a case of the infinite system, one may usually apply the linear response theory where the relevant system is assumed to be coupled with the irrelevant one through the linear coupling, and the latter is usually expressed by a thermal reservoir. As we have discussed in this section, however, one may introduce a concept of "relevant" degrees of freedom in the finite system after requiring an elimination of the linear coupling with leaving only the nonlinear couplings.

\subsection{The scope of the present work}

In order to explore the microscopic dynamics responsible for the macroscopic transport phenomena, a theory of coupled-master equation has been formulated as a general framework for deriving the transport equation, and for clarifying its underlying assumptions(23). In order 
to self-consistently and optimally divide the finite system into a pair of weakly coupled systems, the theory employs the SCC method(60) as mentioned in Sec. 1.4. The self-consistent and optimal separation in the degrees of freedom enables us to study the dissipation mechanisms of large-amplitude relevant motion and nonlinear dynamics between the relevant and irrelevant modes of motion. An important point of using the SCC method(60) for dynamically dividing the total system into two subsystems is a form of the resultant coupling between them, where a linear coupling is eliminated by the maximal decoupling condition imposed by the method.

In this chapter, with the microscopic Hamiltonian, we will discuss how to derive the transport equation from the general theory of coupled-master equation and how to realize the dissipation phenomena in the finite system on the basis of the microscopic dynamics, what kinds of necessary conditions there are in realizing the dissipative process, what kinds of dynamical relations there are between the micro-level and phenomenological-level descriptions, without introducing the any statistical anastz.

It will be clarified(30) that the macroscopic transport equation is obtained from the fully microscopic master equation under the following microscopic conditions: (I) The effects coming from the irrelevant subsystem on the relevant one are taken into account and mainly expressed by an average effect over the irrelevant distribution function. Namely, the fluctuation effects are considered to be sufficiently small and are able to be treated as a perturbation around the path generated by the average Hamiltonian. (II) The irrelevant distribution function has already reached its time-independent stationary state before the main microscopic dynamics responsible for the damping of the relevant motion dominates. As discussed in Sec. 3.1.2, this situation was turned out to be well realized even in the two degrees of freedom system. (III) The time scale of the motion for the irrelevant subsystem is much shorter than that for the relevant one.

The numerical simulations are carried out for a microscopic system composed of the relevant one-degree of freedom system coupled to the irrelevant two-degree of freedom system (described by a classical SU(3) Hamiltonian) through a weak coupling interaction. The novelties of our approach are: (I) the total system is dynamically and optimally divided into the relevant and irrelevant degrees of freedom in a way consistent with the underlying microscopic dynamics for aiming to properly characterize the collective motion; (II) the macroscopic irreversibility is dynamically realized for a finite system rather than introducing any statistical anastz as for infinite system with extremely large number of degrees of freedom. The transport phenomenon will been established numerically(30). It will be also clarified that for the case with a small number degrees of freedom (say, two), the microscopic dephasing mechanism, which is caused by the chaoticity of irrelevant system, is responsible for the energy transfer from the collective system to the environment. Although our numerical simulation by employing the Langevin equation was able to reproduce the macro-level transport phenomenon, it was also clarified that there are substantial differences in the micro-level mechanism between the fully microscopic description and the Langevin description, and in order to reproduce the same results the parameters used in the Langevin equation do not satisfy the fluctuation-dissipation theorem.

Therefore various questions related to the transport phenomenon realized in the finite system on how to understand the differences between the above-mentioned two descriptions, what kinds of other microscopic mechanisms are there besides the dephasing, and when the fluctuation-dissipation theorem comes true etc. are still remained. In the conventional approaches like the Fokker-Planck or Langevin type equations, the irrelevant subsystem is 
always assumed to have a large (even in $\square$ nite) number of degrees of freedom and is placed in a canonically equilibrated state. It is then quite natural to ask whether these problems are caused by a limited number (only two) of degrees of freedom in the irrelevant subsystem considered in our previous work. In order to fill the gap between two and infinite degrees of freedom for the irrelevant subsystem, it is extremely important to study how the microscopic dynamics depends on the number of the degrees of freedom in the irrelevant subsystem.

In this chapter, we will further use a $\beta$-Fermi-Pasta-Ulam $(\beta$-FPU) system representing the irrelevant system, which allows us to change the number of degrees of freedom very conveniently and meanwhile retain the chaoticity of the dynamics of $\beta$-FPU system with the same specific dynamical condition. It will be shown that although the dephasing mechanism is the main mechanism for a case with a small number of degrees of freedom (say, two), the diffusion mechanism will start to play a role as the number of degrees of freedom becomes large (say, eight or more), and, in general, the energy transport process occurs by passing through three distinct stages, such as the dephasing, the statistical relaxation, and the equilibrium regimes. By examining a time evolution of a non-extensive entropy(84), an existence of three regimes will be clearly exhibited.

Exploiting an analytical relation, it will be shown that the energy transport process is described by the generalized Fokker-Planck and Langevin type equation, and a phenomenological fluctuation-dissipation relation is satisfied in a case with relatively large degrees of freedom system. It will be clarified that the irrelevant subsystem with finite number of degrees of freedom can be treated as a heat bath with a finite correlation time, and the statistical relaxation turns out to be an anomalous diffusion, and both the microscopic approach and the conventional phenomenological approach may reach the same level description for the transport phenomena only when the number of irrelevant degrees of freedom becomes very large.

It should be mentioned that a necessity of using a non-extensive entropy for characterizing the damping phenomenon in the finite system is very interesting in connecting the microscopic dynamics and the statistical mechanics, because the non-extensive entropy $(83 ; 84)$ might characterize the non-statistical evolution process more properly than the physical Boltzmann-Gibbs entropy. This might suggest us that the damping mechanism in the finite system is a non-statistical process, where the usual fluctuation-dissipation theorem is not applicable.

We will be able to reach all these goals only within a microscopic classical dynamics of a finite system. The outline of this chapter is as follows. In Sec. 1, we have briefly introduced some background knowledge and motivations of this study. This section is written in a very compact way because I want to pay my most attention on introducing our new progresses what the readers really want to know. The detailed information can be easily found in references. In Sec. 2, we briefly recapitulate the theory of coupled-master equation(23) for the sake of self-containedness. Starting from the most general coupled-master equation, we try to derive the Fokker-Planck and Langevin type equation, by clarifying necessary underlying conditions. Aiming to realize such a physical situation where these conditions are satisfied, in Sec. 3.1, various numerical simulations will be performed for a system where a relevant (collective) harmonic oscillator is coupled with the irrelevant (intrinsic) SU(3) model. After numerically realizing a macro-level transport phenomena, we will try to reproduce it by using a phenomenological Langevin equation, whose potential is derived microscopically. In Sec. 3.2, special emphasis will be put on the effects depending on the number of irrelevant degrees of freedom with a microscopic Hamiltonian where irrelevant system is described by 
a $\beta$-Fermi-Pasta-Ulam ( $\beta$-FPU) system. We will discuss the behavior of the energy transfer process, energy equipartition problem and their dependence on the number of degrees of freedom. The time evolution of entropy by using the nonextensive thermo-dynamics and microscopic dynamics of non-equilibrium transport process will be examined in Sec. 4. In Sec. 5, we will further explore our results in an analytical way with deriving a generalized Fokker-Planck equation and a phenomenological Fluctuation-Dissipation relation, and will discuss the underlying physics. By using the $\beta$-FPU model Hamiltonian, we will further explore how different transport phenomena will appear when the two systems are coupled with linear or nonlinear interactions in Sec. 6. The last section is devoted for summary and discussions.

\section{Theory of coupled-master equations and transport equation of collective motion}

As repeatedly mentioned in Sec. 1, when one intends to understand a dynamics of evolution of a finite Hamiltonian system which connects the macro-level dynamics with the micro-level dynamics, one has to start with how to divide the total system into the weakly coupled relevant (collective or macro $\eta, \eta^{*}$ ) and irrelevant (intrinsic or micro $\xi, \xi^{*}$ ) systems. As an example, the nucleus provides us with a very nice benchmark field because it shows a coexistence of "macroscopic" and "microscopic" effects in association with various "phase transitions", and a mutual relation between "classical" and "quantum" effects related with the macro-level and micro-level variables, respectively. At certain energy region, the nucleus exhibits some statistical aspects which are associated with dissipation phenomena well described by the phenomenological transport equation.

\subsection{Nuclear coupled master equation}

Exploring the microscopic theory of nuclear large-amplitude collective dissipative motion, whose characteristic energy per nucleon is much smaller than the Fermi energy, one may start with the time-dependent Hartree-Fock (TDHF) theory. Since the basic equation of the TDHF theory is known to be formally equivalent to the classical canonical equations of motion (64), the use of the TDHF theory enables us to investigate the basic ingredients of the nonlinear nuclear dynamics in terms of the TDHF trajectories. The TDHF equation is expressed as :

$$
\delta\left\langle\Phi(t)\left|\left(i \frac{\partial}{\partial t}-\hat{H}\right)\right| \Phi(t)\right\rangle=0,
$$

where $|\Phi(t)\rangle$ is the general time-dependent single Slater determinant given by

$$
|\Phi(t)\rangle=\exp \{i \hat{F}\} \mid \Phi_{0}>e^{i E_{0} t}, \quad i \hat{F}=\sum_{\mu i}\left\{f_{\mu i}(t) \hat{a}_{\mu}^{\dagger} \hat{b}_{i}^{\dagger}-f_{\mu i}^{*}(t) \hat{b}_{i} \hat{a}_{\mu}\right\},
$$

where $\left|\Phi_{0}\right\rangle$ denotes a HF stationary state, and $\hat{a}_{\mu}^{\dagger}(\mu=1,2, \ldots, m)$ and $\hat{b}_{i}^{\dagger}(i=1,2, \ldots, n)$ mean the particle- and hole-creation operators with respect to $\left|\Phi_{0}\right\rangle$. The HF Hamiltonian $H$ and the HF energy $E_{0}$ are defined as

$$
H=\langle\Phi(t)|\hat{H}| \Phi(t)\rangle-E_{0}, \quad E_{0}=\left\langle\Phi_{0}|\hat{H}| \Phi_{0}\right\rangle .
$$

With the aid of the self-consistent collective coordinate (SCC) method (60), the whole system can be optimally divided into the relevant (collective) and irrelevant (intrinsic) degrees 
of freedom by introducing an optimal canonical coordinate system called the dynamical canonical coordinate (DCC) system for a given trajectory. That is, the total closed system $\eta \oplus \xi$ is dynamically divided into two subsystems $\eta$ and $\xi$, whose optimal coordinate systems are expressed as $\eta_{a}, \eta_{a}^{*}: a=1, \cdots$ and $\xi_{\alpha}, \xi_{\alpha}^{*}: \alpha=1, \cdots$, respectively. The resulting Hamiltonian in the DCC system is expressed as:

$$
H=H_{\eta}+H_{\xi}+H_{\text {coupl }}
$$

where $H_{\eta}$ depends on the relevant, $H_{\xi}$ on the irrelevant, and $H_{\text {coupl }}$ on both the relevant and irrelevant variables. The TDHF equation (22) can then be formally expressed as a set of canonical equations of motion in the classical mechanics in the TDHF phase space (symplectic manifold), as

$$
i \dot{\eta}_{a}=\frac{\partial H}{\partial \eta_{a}^{*}}, \quad i \dot{\eta}_{a}^{*}=-\frac{\partial H}{\partial \eta_{a}}, \quad i \dot{\xi}_{\alpha}=\frac{\partial H}{\partial \xi_{\alpha}^{*}}, \quad i \dot{\xi}_{\alpha}^{*}=-\frac{\partial H}{\partial \xi_{\alpha}}
$$

Here, it is worthwhile mentioning that the SCC method defines the DCC system so as to eliminate the linear coupling between the relevant and irrelevant subsystems, i.e., the maximal decoupling condition(23) given by Eq. (20),

$$
\left.\frac{\partial H_{\text {coupl }}}{\partial \eta}\right|_{\xi=\xi^{*}=0}=0,
$$

is satisfied. This separation in the degrees of freedom will turn out to be very important for exploring the energy dissipation process and nonlinear dynamics between the collective and intrinsic modes of motion.

The transport, dissipative and damping phenomena appearing in the nuclear system may involve a dynamics described by the wave packet rather than that by the eigenstate. Within the mean-field approximation, these phenomena may be expressed by the collective behavior of the ensemble of TDHF trajectories, rather than the single trajectory. A difference between the dynamics described by the single trajectory and by the bundle of trajectories might be related to the controversy on the effects of one-body and two-body dissipations $(28 ; 40$; $41 ; 65 ; 66)$, because a single trajectory of the Hamilton system will never produce any energy dissipation. Since an effect of the collision term is regarded to generate many-Slater determinants out of the single-Slater determinant, an introduction of the bundle of trajectories is considered to create a very similar situation which is produced by the two-body collision term.

In the classical theory of dynamical system, the order-to-chaos transition is usually regarded as the microscopic origin of an appearance of the statistical state in the finite system. Since one may express the heat bath by means of the infinite number of integrable systems like the harmonic oscillators whose frequencies have the Debye distribution, it may not be a relevant question whether the chaos plays a decisive role for the dissipation mechanism and for the microscopic generation of the statistical state in a case of the infinite system. In the finite system where the large number limit is not secured, the order-to-chaos is expected to play a decisive role in generating some statistical behavior.

To deal with the ensemble of TDHF trajectories, we start with the Liouville equation for the distribution function: 


$$
\begin{aligned}
& \dot{\rho}(t)=-i \mathcal{L} \rho(t), \quad \mathcal{L} * \equiv i\{H, *\}_{P B}, \\
& \rho(t)=\rho\left(\eta(t), \eta(t)^{*}, \xi(t), \xi(t)^{*}\right),
\end{aligned}
$$

which is equivalent to TDHF equation (22). Here the symbol \{\}$_{P B}$ denotes the Poisson bracket. Since we are interested in the time evolution of the bundle of TDHF trajectories, whose bulk properties ought to be expressed by the relevant variables alone, we introduce the reduced distribution functions as

$$
\rho_{\eta}(t)=\operatorname{Tr}_{\xi} \rho(t), \quad \rho_{\xi}(t)=\operatorname{Tr}_{\eta} \rho(t) .
$$

Here, the total distribution function $\rho(t)$ is normalized so as to satisfy the relation

$$
\operatorname{Tr} \rho(t)=1,
$$

where

$$
\begin{gathered}
\operatorname{Tr} \equiv \operatorname{Tr}_{\eta} \operatorname{Tr}_{\xi}, \\
\operatorname{Tr}_{\eta} \equiv \prod_{a} \iint d \eta_{a} d \eta_{a}^{*}, \quad \operatorname{Tr}_{\xi} \equiv \prod_{\alpha} \iint d \xi_{\alpha} d \xi_{\alpha}^{*} .
\end{gathered}
$$

With the aid of the reduced distribution functions $\rho_{\eta}(t)$ and $\rho_{\xi}(t)$, one may decompose the Hamiltonian in Eq. (25) into the form

$$
\begin{aligned}
& H=H_{\eta}+H_{\xi}+H_{\text {coupl }} \\
& =H_{\eta}+H_{\eta}(t)+H_{\xi}+H_{\xi}(t)+H_{\Delta}(t)-E_{0}(t), \\
& H_{\eta}(t) \equiv \operatorname{Tr}_{\xi} H_{\text {coupl }} \rho_{\xi}(t) \text {, } \\
& H_{\xi}(t) \equiv \operatorname{Tr}_{\eta} H_{\text {coupl }} \rho_{\eta}(t) \text {, } \\
& H_{\text {aver }}(t) \equiv H_{\eta}(t)+H_{\xi}(t), \\
& E_{0}(t) \equiv \operatorname{Tr} H_{\text {coupl }} \rho(t), \\
& H_{\Delta}(t) \equiv H_{\text {coupl }}-H_{\text {aver }}(t)+E_{0}(t) .
\end{aligned}
$$

The corresponding Liouvillians are defined as

$$
\begin{gathered}
\mathcal{L}_{\eta} * \\
\mathcal{L}_{\eta}(t) * i\left\{H_{\eta}, *\right\}_{P B} \\
\mathcal{L}_{\xi^{*}} \equiv i\left\{H_{\eta}(t), *\right\}_{P B} \\
\left.\mathcal{L}_{\xi}(t) * \equiv i\left\{H_{\xi}, *\right\}_{P B}(t), *\right\}_{P B} \\
\mathcal{L}_{\text {coupl }} * \equiv i\left\{H_{\text {coupl }}, *\right\}_{P B} \\
\mathcal{L}_{\Delta}(t) * \equiv i\left\{H_{\Delta}(t), *\right\}_{P B}
\end{gathered}
$$


Through above optimal division of the total system into the relevant and irrelevant degrees of freedom, one can treat the two subsystems in a very parallel way. Since one intends to explore how the statistical nature appears as a result of the microscopic dynamics, one should not introduce any statistical ansatz for the irrelevant distribution function $\rho_{\xi}$ by hand, but should properly take account of its time evolution. By exploiting the time-dependent projection operator method (67), one may decompose the distribution function into a separable part and a correlated one as

$$
\begin{aligned}
& \rho(t)=\rho_{s}(t)+\rho_{c}(t), \\
& \rho_{\mathcal{S}}(t) \equiv P(t) \rho(t)=\rho_{\eta}(t) \rho_{\xi}(t), \\
& \rho_{\mathcal{c}}(t) \equiv(1-P(t)) \rho(t),
\end{aligned}
$$

where $P(t)$ is the time-dependent projection operator defined by

$$
P(t) \equiv \rho_{\eta}(t) \operatorname{Tr}_{\eta}+\rho_{\xi}(t) \operatorname{Tr}_{\xi}-\rho_{\eta}(t) \rho_{\xi}(t) \operatorname{Tr}_{\eta} \operatorname{Tr}_{\xi} .
$$

From the Liouville equation (28), one gets

$$
\begin{aligned}
& \dot{\rho}_{\mathcal{S}}(t)=-i P(t) \mathcal{L} \rho_{s}(t)-i P(t) \mathcal{L} \rho_{c}(t) \\
& \dot{\rho}_{c}(t)=-i(1-P(t)) \mathcal{L} \rho_{s}(t)-i(1-P(t)) \mathcal{L} \rho_{c}(t) .
\end{aligned}
$$

By introducing the propagator

$$
g\left(t, t^{\prime}\right) \equiv \operatorname{Texp}\left\{-i \int_{t^{\prime}}^{t}[1-P(\tau)] \mathcal{L} d \tau\right\}
$$

where $T$ denotes the time ordering operator, one obtains the master equation for $\rho_{s}(t)$ as

$$
\begin{aligned}
\dot{\rho}_{S}(t)= & -i P(t) \mathcal{L} \rho_{S}(t)-i P(t) \mathcal{L} g\left(t, t_{I}\right) \rho_{\mathcal{C}}\left(t_{I}\right) \\
& -\int_{t_{I}}^{t} d t^{\prime} P(t) \mathcal{L} g\left(t, t^{\prime}\right)\left\{1-P\left(t^{\prime}\right)\right\} \mathcal{L} \rho_{S}\left(t^{\prime}\right),
\end{aligned}
$$

where $t_{I}$ stands for an initial time. In the conventional case, one usually takes an initial condition

$$
\rho_{c}\left(t_{I}\right)=0, \quad \text { i.e., } \quad \rho\left(t_{I}\right)=\rho_{\eta}\left(t_{I}\right) \cdot \rho_{\xi}\left(t_{I}\right) .
$$

That is, there are no correlation at the initial time. According to this assumption, one may eliminate the second term on the rhs of Eq. (39). In our present general case, however, we have to retain this term, which allows us to evaluate the memory effects by starting from various time $t_{I}$.

With the aid of some properties of the projection operator $P(t)$ defined in Eq. (36) and the relations

$$
\operatorname{Tr}_{\eta} \mathcal{L}_{\eta}=0, \quad \operatorname{Tr}_{\xi} \mathcal{L}_{\xi}=0, \quad \operatorname{Tr}_{\eta} \mathcal{L}_{\eta}(t)=0, \quad \operatorname{Tr}_{\xi} \mathcal{L}_{\xi}(t)=0,
$$




$$
\begin{array}{ll}
\mathcal{L}_{\eta} * \equiv i\left\{H_{\eta}, *\right\}_{P B}, & \mathcal{L}_{\eta}(t) * \equiv i\left\{H_{\eta}(t), *\right\}_{P B}, \\
\mathcal{L}_{\xi} * i\left\{H_{\xi}, *\right\}_{P B}, & \mathcal{L}_{\xi}(t) * \equiv i\left\{H_{\xi}(t), *\right\}_{P B},
\end{array}
$$

as is easily proved, the Liouvillian $\mathcal{L}$ appearing inside the time integration in Eq. (39) is replaced by $\mathcal{L}_{\text {coupl }}$ defined by $\mathcal{L}_{\text {coupl }} *=\left\{H_{\text {coupl }}, *\right\}_{P B}$ and Eq. (39) is reduced to

$$
\begin{aligned}
\dot{\rho}_{S}(t)= & -i P(t) \mathcal{L} \rho_{S}(t)-i P(t) \mathcal{L} g\left(t, t_{I}\right) \rho_{c}\left(t_{I}\right) \\
& -\int_{t_{I}}^{t} d t^{\prime} P(t) \mathcal{L}_{\Delta}(t) g\left(t, t^{\prime}\right)\left\{1-P\left(t^{\prime}\right)\right\} \mathcal{L}_{\Delta}\left(t^{\prime}\right) \rho_{s}\left(t^{\prime}\right),
\end{aligned}
$$

Expressing $\rho_{s}(t)$ and $P(t)$ in terms of $\rho_{\eta}(t)$ and $\rho_{\xi}(t)$, and operating $\operatorname{Tr}_{\eta}$ and $\operatorname{Tr}_{\xi}$ on Eq. (39), one obtains a coupled master equation

$$
\begin{aligned}
\dot{\rho}_{\eta}(t)= & -i\left[\mathcal{L}_{\eta}+\mathcal{L}_{\eta}(t)\right] \rho_{\eta}(t)-i \operatorname{Tr}_{\xi}\left[\mathcal{L}_{\eta}+\mathcal{L}_{\text {coupl }}\right] g\left(t, t_{I}\right) \rho_{c}\left(t_{I}\right) \\
& -\int_{t_{I}}^{t} d \tau \operatorname{Tr}_{\xi} \mathcal{L}_{\Delta}(t) g(t, \tau) \mathcal{L}_{\Delta}(\tau) \rho_{\eta}(\tau) \rho_{\xi}(\tau), \\
\dot{\rho}_{\xi}(t)= & -i\left[\mathcal{L}_{\xi}+\mathcal{L}_{\xi}(t)\right] \rho_{\xi}(t)-i \operatorname{Tr}_{\eta}\left[\mathcal{L}_{\xi}+\mathcal{L}_{\text {coupl }}\right] g\left(t, t_{I}\right) \rho_{\mathcal{c}}\left(t_{I}\right) \\
& -\int_{t_{I}}^{t} d \tau \operatorname{Tr}_{\eta} \mathcal{L}_{\Delta}(t) g(t, \tau) \mathcal{L}_{\Delta}(\tau) \rho_{\eta}(\tau) \rho_{\xi}(\tau),
\end{aligned}
$$

where $\mathcal{L}_{\Delta}(t) * \equiv\left\{H_{\Delta}(t), *\right\}_{P B}$. The first (instantaneous) term describes the reversible motion of the relevant and irrelevant systems while the second and third terms bring on irreversibility. The coupled master equation (43) is still equivalent to the original Liouville equation (28) and can describe a variety of dynamics of the bundle of trajectories. In comparison with the usual time-independent projection operator method of Nakajima-Zwanzig (68) (69) where the irrelevant distribution function $\rho_{\xi}$ is assumed to be a stationary heat bath, the present coupled-master equation (43) is rich enough to study the microscopic origin of the large-amplitude dissipative motion.

\subsection{Dynamical response and correlation functions}

As was discussed in Sec. 3.1.2 and Ref.(22), a bundle of trajectories even in the two degrees of freedom system may reach a statistical object. In this case, it is reasonable to assume that the effects on the relevant system coming from the irrelevant one are mainly expressed by an averaged effect over the irrelevant distribution function (Assumption). Namely, the effects due to the fluctuation part $H_{\Delta}(t)$ are assumed to be much smaller than those coming from $H_{\text {aver }}(t)$. Under this assumption, one may introduce the mean-eld propagator

$$
\begin{gathered}
g_{m f}\left(t, t^{\prime}\right)=\operatorname{Texp}\left\{-i \int_{t^{\prime}}^{t}[1-P(\tau)] \mathcal{L}^{m f}(\tau) d \tau\right\}, \\
\mathcal{L}^{m f}(t)=\mathcal{L}_{\eta}^{m f}(t)+\mathcal{L}_{\xi}^{m f}(t),
\end{gathered}
$$




$$
\begin{aligned}
& \mathcal{L}_{\eta}^{m f}(t) \equiv \mathcal{L}_{\eta}+\mathcal{L}_{\eta}(t), \\
& \mathcal{L}_{\xi}^{m f}(t) \equiv \mathcal{L}_{\xi}+\mathcal{L}_{\tilde{\xi}}(t),
\end{aligned}
$$

which describes the major time evolution of the system, while the fluctuation part is regarded as a perturbation. By further introducing the following propagators given by

$$
\begin{aligned}
& G_{m f}\left(t, t^{\prime}\right) \equiv \operatorname{Texp}\left\{-i \int_{t^{\prime}}^{t} \mathcal{L}^{m f}(\tau) d \tau\right\}=G_{\eta}\left(t, t^{\prime}\right) G_{\xi}\left(t, t^{\prime}\right), \\
& G_{\eta}\left(t, t^{\prime}\right) \equiv \operatorname{Texp}\left\{-i \int_{t^{\prime}}^{t} \mathcal{L}_{\eta}^{m f}(\tau) d \tau\right\}, \\
& G_{\xi}\left(t, t^{\prime}\right) \equiv \operatorname{Texp}\left\{-i \int_{t^{\prime}}^{t} \mathcal{L}_{\xi}^{m f}(\tau) d \tau\right\},
\end{aligned}
$$

one may prove that there holds a relation

$$
g_{m f}(t, \tau) \mathcal{L}_{\Delta}(\tau) \rho_{\eta}(\tau) \rho_{\xi}(\tau)=G_{m f}(t, \tau) \mathcal{L}_{\Delta}(\tau) \rho_{\eta}(\tau) \rho_{\xi}(\tau) .
$$

The coupling interaction is generally expressed as

$$
H_{\text {coupl }}(\eta, \xi)=\sum_{l} A^{l}(\eta) B^{l}(\xi)
$$

For simplicity, we hereafter discard the summation $l$ in the coupling. By introducing the generalized two-time correlation and response functions, which have been called dynamical correlation and response functions in Ref. (21), through

$$
\begin{aligned}
\phi(t, \tau) & \equiv \operatorname{Tr}_{\xi} G_{\xi}(\tau, t) B \cdot\left(B-<B>_{t}\right) \rho_{\xi}(\tau), \\
\chi(t, \tau) & \equiv \operatorname{Tr}_{\xi}\left\{G_{\xi}(\tau, t) B, B\right\}_{P B} \rho_{\xi}(\tau),
\end{aligned}
$$

with $<B>_{t} \equiv \operatorname{Tr}_{\xi} B \rho_{\xi}(t)$, the master equation in Eq.(43) for the relevant degree of freedom is expressed as

$$
\begin{aligned}
\dot{\rho}_{\eta}(t)= & -i\left[\mathcal{L}_{\eta}+\mathcal{L}_{\eta}(t)\right] \rho_{\eta}(t)-i \operatorname{Tr}_{\xi}\left[\mathcal{L}_{\eta}+\mathcal{L}_{\text {coupl }}\right] g\left(t, t_{I}\right) \rho_{\mathcal{c}}\left(t_{I}\right) \\
& +\int_{0}^{t-t_{I}} d \tau \chi(t, t-\tau)\left\{A, G_{\eta}(t, t-\tau)\left(A-<A>_{t-\tau}\right) \rho_{\eta}(t-\tau)\right\}_{P B} \\
& +\int_{0}^{t-t_{I}} d \tau \phi(t, t-\tau)\left\{A, G_{\eta}(t, t-\tau)\left\{A, \rho_{\eta}(t-\tau)\right\}_{P B}\right\}_{P B}{ }^{\prime}
\end{aligned}
$$

with $<A>_{t} \equiv \operatorname{Tr}_{\eta} A \rho_{\eta}(t)$. Here, it should be noted that the whole system is developed exactly up to $t_{I}$. In order to make Eq.(49) applicable, $t_{I}$ should be taken to be very close to a time when the irrelevant system approaches very near to its stationary state (, i.e., the irrelevant 
system is very near to the statistical state where one may safely make the assumption to be stated in next subsection). In order to analyze what happens in the microscopic system which is situated far from its stationary states, one has to study $\chi\left(t_{I}, t_{I}-\tau\right)$ and $\phi\left(t_{I}, t_{I}-\tau\right)$ by changing $t_{I}$. Since both $\chi\left(t_{I}, t_{I}-\tau\right)$ and $\phi\left(t_{I}, t_{I}-\tau\right)$ are strongly dependent on $t_{I}$, it is not easy to explore the dynamical evolution of the system far from the stationary state. So as to make Eq.(49) applicable, we will exploit the further assumptions.

\subsection{Macroscopic transport equation}

In this subsection, we discuss how the macroscopic transport equation is obtained from the fully microscopic master equation (49) by clearly itemizing necessary microscopic conditions.

Condition I Suppose the relevant distribution function $\rho_{\eta}(t-\tau)$ inside the time integration in Eq. (49) evolves through the mean-field Hamiltonian $H_{\eta}+H_{\eta}(t)^{1}$. Namely, $\rho_{\eta}(t-\tau)$ inside the integration is assumed to be expressed as $\rho_{\eta}(t)=G_{\eta}(t, t-\tau) \rho_{\eta}(t-\tau)$, so that Eq.(49) is reduced to

$$
\begin{aligned}
\dot{\rho}_{\eta}(t)= & -i\left[\mathcal{L}_{\eta}+\mathcal{L}_{\eta}(t)\right] \rho_{\eta}(t)-i \operatorname{Tr}_{\xi}\left[\mathcal{L}_{\eta}+\mathcal{L}_{\text {coupl }}\right] g\left(t, t_{I}\right) \rho_{c}\left(t_{I}\right) \\
& +\int_{0}^{t-t_{I}} d \tau \chi(t, t-\tau)\left\{A, G_{\eta}(t, t-\tau)\left(A-<A>_{t-\tau}\right) \cdot \rho_{\eta}(t)\right\}_{P B} \\
& +\int_{0}^{t-t_{I}} d \tau \phi(t, t-\tau)\left\{A,\left\{G_{\eta}(t, t-\tau) A, \rho_{\eta}(t)\right\}_{P B}\right\}_{P B} .
\end{aligned}
$$

This condition is equivalent to Assumption discussed in the previous subsection, because the fluctuation effects are sufficiently small and are able to be treated as a perturbation around the path generated by the mean-field Hamiltonian $H_{\eta}+H_{\eta}(t)$, and are sufficient to be retained in Eq. (50) up to the second order.

Condition II Suppose the irrelevant distribution function $\rho_{\xi}(t)$ has already reached its time-independent stationary state $\rho_{\xi}\left(t_{0}\right)$. According to our previous paper(22), this situation is able to be well realized even in the 2-degrees of freedom system. Under this assumption, the relevant mean-field Liouvillian $\mathcal{L}_{\eta}+\mathcal{L}_{\eta}(t)$ becomes a time independent object. Under this assumption, a time ordered integration in $G_{\eta}\left(t, t^{\prime}\right)$ defined in Eq. (44) is performed and one may introduce

$$
G_{\eta}(t, t-\tau) \approx G_{\eta}(\tau) \equiv \exp \left\{-i \mathcal{L}_{\eta}^{m f} \tau\right\}, \quad \mathcal{L}_{\eta}^{m f} \equiv \mathcal{L}_{\eta}+\mathcal{L}_{\eta}\left(t_{0}\right),
$$

where $t_{0}$ denotes a time when the irrelevant system has reached its stationary state.

Condition III Suppose the irrelevant time scale is much shorter than the relevant time scale. Under this assumption, the response $\chi(t, t-\tau)$ and correlation functions $\phi(t, t-\tau)$ are regarded to be independent of the time $t$, because $t$ in Eq.(50) is regarded to describe a very slow time evolution of the relevant motion. By introducing an approximate one-time response and correlation functions

$$
\chi(\tau) \approx \chi(t, t-\tau), \quad \phi(\tau) \approx \phi(t, t-\tau),
$$

\footnotetext{
${ }^{1}$ The same assumption has been introduced in a case of the linear coupling(27).
} 
one may get

$$
\begin{aligned}
\dot{\rho}_{\eta}(t)= & -i\left[\mathcal{L}_{\eta}+\mathcal{L}_{\eta}(t)\right] \rho_{\eta}(t)-i \operatorname{Tr}_{\xi}\left[\mathcal{L}_{\eta}+\mathcal{L}_{\text {coupl }}\right] g\left(t, t_{I}\right) \rho_{c}\left(t_{I}\right) \\
& +\int_{0}^{\infty} d \tau \chi(\tau)\left\{A, \exp \left\{-i \mathcal{L}_{\eta}^{m f} \tau\right\}\left(A-<A>_{t-\tau}\right) \cdot \rho_{\eta}(t)\right\}_{P B} \\
& +\int_{0}^{\infty} d \tau \phi(\tau)\left\{A,\left\{\exp \left(-i \mathcal{L}_{\eta}^{m f} \tau\right) A, \rho_{\eta}(t)\right\}_{P B}\right\}_{P B} .
\end{aligned}
$$

This condition is different from the diabatic condition(17; 19), where the ratio between the characteristic times of the irrelevant degrees of freedom and of the relevant one is considered arbitrary small. However this condition is only partly satisfied for the most realistic cases. The dissipation is necessarily connected to some degree of chaoticity of the overall dynamics of the system(28).

Here it should be noted that such one-time response and correlation functions are still different from the usual ones introduced in the LRT where the concepts of linear coupling and of heat bath are adopted. Under the same assumption, the upper limit of the integration $t-t_{I}$ in Eq. (53) can be extended to the infinity, because the $\chi(\tau)$ and $\phi(\tau)$ are assumed to be very fast damping functions when it is measured in the relevant time scale.

Here, one may introduce the susceptibility $\zeta(t)$

$$
\zeta(t)=\int_{0}^{t} d \tau \chi(\tau), \quad \zeta(0)=0 .
$$

Defining $\zeta \equiv \zeta(\infty)$, one may further introduce another dynamical function $c(t)$ :

$$
\zeta(t)=[1-c(t)] \zeta, \quad \text { with } \quad c(0)=1, \quad c(\infty)=0,
$$

which satisfies the following relation

$$
\chi(t)=\frac{\partial \zeta(t)}{\partial t}=-\zeta \frac{\partial c(t)}{\partial t}
$$

Inserting Eq. (56) into Eq. (53) and integrating by part, one gets

$$
\begin{aligned}
\dot{\rho}_{\eta}(t)= & -i\left[\mathcal{L}_{\eta}+\mathcal{L}_{\eta}(t)\right] \rho_{\eta}(t)-i \operatorname{Tr}_{\xi}\left[\mathcal{L}_{\eta}+\mathcal{L}_{\text {coupl }}\right] g\left(t, t_{I}\right) \rho_{c}\left(t_{I}\right) \\
& +\zeta\left\{A,\left(A-<A>_{t}\right) \cdot \rho_{\eta}(t)\right\}_{P B} \\
& +\zeta \int_{0}^{\infty} d \tau c(\tau)\left\{A, \frac{d}{d \tau}\left(\exp \left(-i \mathcal{L}_{\eta}^{m f} \tau\right)\left(A-<A>_{t}\right)\right) \cdot \rho_{\eta}(t)\right\}_{P B} \\
& +\int_{0}^{\infty} d \tau \phi(\tau)\left\{A,\left\{\exp \left(-i \mathcal{L}_{\eta}^{m f} \tau\right) A, \rho_{\eta}(t)\right\}_{P B}\right\}_{P B} .
\end{aligned}
$$

This equation is a Fokker-Planck type equation. The first term on the right-hand side of Eq. (57) represents the contribution from the mean-field part, and the second term a contribution 
from the correlated part of the distribution function at time $t_{I}$. The last three terms represent contribution from the dynamical fluctuation effects $H_{\Delta}$. The friction as well as fluctuation terms are supposed to emerge as a result of those three terms. We will discuss the role of each term with our numerical simulation in the next section.

At the end of this subsection, let us discuss how to obtain the Langevin equation from our fully microscopic coupled master equation, because it has been regarded as a final goal of the microscopic or dynamical approaches to justify the phenomenological approaches. For a sake of simplicity, let us discuss a case where the interaction between relevant and irrelevant degrees of freedom has the following linear form,

$$
\begin{aligned}
& H_{\text {coupl }}=\lambda Q \sum_{i} q_{i}, \quad \text { i.e. } \quad A=\sqrt{\lambda} Q, \quad B=\sqrt{\lambda} \sum_{i} q_{i}, \\
& Q=\frac{1}{\sqrt{2}}\left(\eta+\eta^{*}\right), \quad P=\frac{i}{\sqrt{2}}\left(\eta^{*}-\eta\right), \\
& q_{i}=\frac{1}{\sqrt{2}}\left(\xi_{i}+\xi_{i}^{*}\right), \quad p_{i}=\frac{i}{\sqrt{2}}\left(\xi_{i}^{*}-\xi_{i}\right) .
\end{aligned}
$$

Here, we assume that the relevant system consists of one degree of freedom described by $P, Q$. Even though we apply the linear coupling form, the generalization for the case with more general nonlinear coupling is straightforward. In order to evaluate Eq. (57), one has to calculate

$$
Q(\tau)=\exp \left(-i \mathcal{L}_{\eta}^{m f} \tau\right) Q
$$

where $Q(\tau)$ is a phase space image of $Q$ through the backward evolution. Thus the Poisson bracket $\left\{Q(\tau), \rho_{\eta}(t)\right\}_{P B}$ in Eq. (57) is expressed as

$$
\left\{Q(\tau), \rho_{\eta}(t)\right\}_{P B}=\frac{\partial Q(\tau)}{\partial Q} \frac{\partial \rho_{\eta}(t)}{\partial P}-\frac{\partial Q(\tau)}{\partial P} \frac{\partial \rho_{\eta}(t)}{\partial Q} .
$$

By introducing the following quantities,

$$
\begin{aligned}
& \alpha_{1}(P, Q) \equiv \lambda \int_{0}^{\infty} d \tau \phi(\tau) \frac{\partial Q(\tau)}{\partial Q}, \\
& \alpha_{2}(P, Q) \equiv-\lambda \int_{0}^{\infty} d \tau \phi(\tau) \frac{\partial Q(\tau)}{\partial P}, \\
& \beta(P, Q) \equiv \lambda \zeta \int_{0}^{\infty} d \tau c(\tau) \frac{\partial Q(\tau)}{\partial \tau},
\end{aligned}
$$

Eq. (57) is reduced to

$$
\begin{aligned}
\dot{\rho}_{\eta}(t)= & -i \operatorname{Tr}_{\xi}\left[\mathcal{L}_{\eta}+\mathcal{L}_{\text {coupl }}\right] g\left(t, t_{I}\right) \rho_{\mathcal{c}}\left(t_{I}\right) \\
& +\left\{-i\left(\mathcal{L}_{\eta}+\mathcal{L}_{\eta}(t)\right)+\lambda \zeta\left(Q-\langle Q\rangle_{t}\right) \frac{\partial}{\partial P}\right. \\
& \left.+\frac{\partial}{\partial P} \beta(P, Q)+\frac{\partial}{\partial P} \alpha_{1}(P, Q) \frac{\partial}{\partial P}+\frac{\partial}{\partial P} \alpha_{2}(P, Q) \frac{\partial}{\partial Q}\right\} \rho_{\eta}(t)
\end{aligned}
$$


As discussed in Ref. (26), Eq. (62) results in the Langevin equation with a form

$$
\ddot{Q}=-\frac{1}{m} \frac{\partial U(Q)}{\partial x}-\gamma \dot{Q}+f(t)
$$

by introducing a concept of mechanical temperature.

The above derivation of the Langevin equation is still too formal to be applicable for the general cases. However it might be naturally expected that the Conditions I, II and III are met in the actual dynamical processes.

\section{Dynamic realization of transport phenomenon in finite system}

In order to study the dissipation process microscopically, it is inevitable to treat a system with more than two degrees of freedom, which is able to be divided into two weakly coupled subsystems: one is composed of at least two degrees of freedom and is regarded as an irrelevant system, whereas the rest is considered as a relevant system. The system with two degrees of freedom is too simple to assign the relevant degree of freedom nor to discuss its dissipation, because the chaotic or statistical state can be realized by a system with at least two degrees of freedom.

\subsection{The case of the system with three degrees of freedom}

\subsubsection{Description of the microscopic system}

The system considered in our numerical calculation is composed of a collective degree of freedom coupled to intrinsic degrees of freedom through weak interaction, which simulates a nuclear system. The collective system describing, e.g., the giant resonance is represented by the harmonic oscillator given by

$$
H_{\eta}(q, p)=\frac{p^{2}}{2 M}+\frac{1}{2} M \omega^{2} q^{2}
$$

and the intrinsic system mimicking the hot nucleus is described by the modified SU(3) model Hamiltonian (70) given by

$$
\hat{H}=\sum_{i=0}^{2} \epsilon_{i} \hat{K}_{i i}+\frac{1}{2} \sum_{i=1}^{2} V_{i}\left\{\hat{K}_{i 0} \hat{K}_{i 0}+\text { h.c. }\right\} ; \quad \hat{K}_{i j}=\sum_{m=1}^{N} C_{i m}^{\dagger} C_{j m}
$$

where $C_{i m}^{+}$and $C_{i m}$ represent the fermion creation and annihilation operators. There are three $\mathrm{N}$-fold degenerate levels with $\epsilon_{0}<\epsilon_{1}<\epsilon_{2}$. In the case with an even $\mathrm{N}$ particle system, the TDHF theory gives a classical Hamiltonian with two degrees of freedom as

$$
\begin{aligned}
H_{\xi}\left(q_{1}, p_{1}, q_{2}, p_{2}\right)= & \frac{1}{2}\left(\epsilon_{1}-\epsilon_{0}\right)\left(q_{1}^{2}+p_{1}^{2}\right)+\frac{1}{2} V_{1}(N-1)\left(q_{1}^{2}-p_{1}^{2}\right) \\
& +\frac{1}{2}\left(\epsilon_{2}-\epsilon_{0}\right)\left(q_{2}^{2}+p_{2}^{2}\right)+\frac{1}{2} V_{2}(N-1)\left(q_{2}^{2}-p_{2}^{2}\right) \\
& -\frac{N-1}{4 N} V_{1}\left(q_{1}^{4}-p_{1}^{4}\right)-\frac{N-1}{4 N} V_{2}\left(q_{2}^{4}-p_{2}^{4}\right) \\
& +\frac{N-1}{4 N}\left[-V_{1}\left(q_{1}^{2}-p_{1}^{2}\right)\left(q_{2}^{2}+p_{2}^{2}\right)-V_{2}\left(q_{1}^{2}+p_{1}^{2}\right)\left(q_{2}^{2}-p_{2}^{2}\right)\right] .
\end{aligned}
$$


In our numerical calculation, the used parameters are $M=18.75, \omega^{2}=0.0064, \epsilon_{0}=0, \epsilon_{1}=1, \epsilon_{2}=2$, $\mathrm{N}=30$ and $\mathrm{V}_{i}=-0.07$. In this case, the collective time scale $\tau_{c o l}$ characterized by the harmonic oscillator in Eq. (64) and the intrinsic time scale $\tau_{\text {in }}$ characterized by the harmonic part of the intrinsic Hamiltonian in Eq.(66) satisfies a relation $\tau_{c o l} \sim 10 \tau_{\text {in }}$.

For the coupling interaction, we use the following nonlinear interaction given by

$$
H_{\text {coupl }}=\lambda\left(q-q_{0}\right)^{2} \sum_{i=1}^{2}\left\{q_{i}^{2}+p_{i}^{2}\right\} .
$$

A physical meaning of introducing a quantity $q_{0}$ in Eq. (67) will be discussed at the end of this subsection as well as the next subsection.

In performing the numerical simulation, the time evolution of the distribution function $\rho(t)$ is evaluated by using the pseudo-particle method as:

$$
\rho(t)=\frac{1}{N_{p}} \sum_{n=1}^{N_{p}} \prod_{i=1}^{2} \delta\left(q_{i}-q_{i, n}(t)\right) \delta\left(p_{i}-p_{i, n}(t)\right) \cdot \delta\left(q-q_{n}(t)\right) \delta\left(p-p_{n}(t)\right),
$$

where $N_{p}$ means the total number of pseudo-particles. The distribution function in Eq. (68) defines an ensemble of the system, each member of which is composed of a collective degree of freedom coupled to a single intrinsic trajectory. The collective coordinates $q_{n}(t)$ and $p_{n}(t)$, and the intrinsic coordinates $q_{i, n}(t)$ and $p_{i, n}(t)$ determine a phase space point of the $\mathrm{n}$-th pseudo-particle at time $t$, whose time dependence is described by the canonical equations of motion given by

$$
\begin{aligned}
& \dot{q}=\frac{\partial H}{\partial p}, \quad \dot{p}=-\frac{\partial H}{\partial q} \\
& \dot{q}_{i}=\frac{\partial H}{\partial p_{i}}, \quad \dot{p}_{i}=-\frac{\partial H}{\partial q_{i}}, \quad\{i=1,2\} \\
& H \equiv H_{\eta}(q, p)+H_{\xi}\left(q_{1}, p_{1}, q_{2}, p_{2}\right)+H_{\text {coupl }}
\end{aligned}
$$

We use the fourth order simplectic Runge-Kutta method(75; 76) for integrating the canonical equations of motion and $N_{p}$ is chosen to be 10,000 . The initial condition for the intrinsic distribution function is given by a uniform distribution in a tiny region of the stochastic sea as stated in Ref. (22). That for the collective distribution function is given by the $\delta$ function centered at $q(0)=0$ and $p(0), p(0)$ being defined by a given collective energy $E_{\eta}$ together with $q(0)=0$. The distribution function in Eq. (68) defines an ensemble of the system, each member of which is composed of a collective degree of freedom coupled to a single intrinsic trajectory.

In our numerical simulation, the coupling interaction is not activated at an initial stage. In the beginning, the coupling between the collective and intrinsic systems is switch off, and they evolve independently. Namely, the collective system evolves regularly, whereas, as discussed in the subsection 3.1.2, the intrinsic system tends to reach its time-independent stationary state (chaotic object). After the statistical state has been realized in the intrinsic system, the coupling interaction is activated. A quantity $q_{0}$ in Eq.(67) denotes a value of the collective trajectory $q$ at the switch on time. A purpose of introducing $q_{0}$ is to insert the coupling adiabatically, and to conserve the total energy before and after the switch on time. (Hereafter, $\tau_{s w}$ denotes the 
moment when the interaction is switch on, and in our numerical calculation $\tau_{s w}$ is set to be $\left.\tau_{s w}=12 \tau_{c o l}\right)$.

Here it is worthwhile to discuss why we let the two systems evolve independently at the initial stage. As is well known, the ergodic and irreversible property of the intrinsic system is assumed in the conventional approach, and the intrinsic system for the in $\sqcap$ nite system is usually represented by the time independent canonical ensemble. In the nite system, however, one has to explore whether or not the intrinsic system tends to reach such a state that is effectively replaced by a statistical object, how it evolves after the coupling interaction is switch on, and what its final state looks like.

As is discussed at the end of the subsection 2.2, it is not easy to apply Eq. (49) for analyzing what happens in the dynamical microscopic system which is in the general situation. Our present primarily aim is to microscopically generate such a transport phenomenon that might be understood in terms of the Langevin equation. Namely, we have to construct such a microscopic situation that seems to satisfy the Condition I, II and III discussed in subsection 2.3. In this context, we firstly let the intrinsic system reach a chaotic situation in a dynamical way, till the ergodic and irreversible property are well realized dynamically. In the next subsection, it will be shown that above microscopic situation is indeed realized dynamically for the intrinsic system (66).

\subsubsection{Dynamic realization of statistical state in finite system}

It is not a trivial discussion how to dynamically characterize the statistical state in the finite system. Even though the Hamilton system shows chaotic situation and the Lyapunov exponent has a positive value everywhere in the phase space, there still remain a lot of questions, such as, whether or not one may substantiate statistical state in the way dynamical chaos is structured in real Hamiltonian system, how the real macroscopic motion looks like in such system, whether or not there are some difficulties of using the properties of dynamical chaos as a source of randomness, whether or not there is difference between real Hamiltonian chaos and a conventional understanding of the laws of statistical physics and whether or not the system dynamically reaches some statistical object. It is certainly interesting question especially for the nuclear physics to explore the relation between the dynamical definition of the statistical state and the static definition of it. The former definition will be discussed in the following, whereas the latter definition is usually given by employing a concept of "temperature" like

$$
\rho=e^{-\beta H}, \quad \beta=\frac{1}{k T} .
$$

Even in the nuclear system, there are many phenomena well explained by using the concept of temperature. To make the discussion simple, we treat the Hamilton system given in Eq. (66). In Fig. 4, the Poincaré section for the case with $N=30, \epsilon_{0}=0, \epsilon_{1}=1, \epsilon_{2}=2$, $V_{1}=V_{2}=-0.07$ and $E=40$ is illustrated. From this figure, one may see that the phase space is dominated by a chaotic sea, with some remnants of KAM torus(71). The toughness of the torus structure is a quite general property in the Hamilton system. Since the KAM torus means an existence of a very sticky motion which travels around the torus for a quite long time, one might expect a very long correlation time which would prevent us from introducing some statistical objects.

As is well known, the nearest-neighbor level-spacing statistics of the quantum system is well described by the GOE, when the phase space of its classical correspondent is covered by a chaotic sea(39). Here it should be remembered that the GOE is derived under the 
assumption that the matrix elements of Hamiltonian should be representation independent(72). This assumption would be considered to be a statistical ansatz introduced for the quantum system. Let us consider the classical analogue of the quantum concept of representation independence. We repeatedly point out an importance of the choice of coordinate system, because it has shown to be very useful in exploring how simply the trajectory under discussion is described, how to obtain its approximate constants of motion, how optimally the total system is divided into the relevant and irrelevant subsystems for a given trajectory and how analytically one may understand an exceedingly rich structure of the phase space. In a case of the chaotic situation where no constants of motion exist except for the total energy, however, there should be no dynamical reason to select some specific coordinate system. In other words, the classical statistical state is expected to be characterized by the coordinate system independence.

The coordinate system $\left\{q_{1}, p_{1}, q_{2}, p_{2}\right\}$ used in describing the Hamilton system in Eq. (66) just corresponds to the maximal-decoupled coordinate system, because it satisfies the maximum-decoupling condition (27). The coordinate system $\left\{q_{1}, p_{1}, q_{2}, p_{2}\right\}$ is identified to be the optimum coordinate system, when an amplitude of the trajectory is sufficiently small and the harmonic term in Eq. (66) is dominated. When the amplitude becomes large, there appears such a situation for the bundle of trajectories, where the following relations are fulfilled,

$$
\begin{aligned}
& \left.<q_{i}\right\rangle_{t}<\sqrt{\left.\left\langle q_{i}^{2}-<q_{i}\right\rangle_{t}^{2}\right\rangle_{t^{\prime}}} \\
& \left.<p_{i}\right\rangle_{t}<\sqrt{\left.\left\langle p_{i}^{2}-<p_{i}\right\rangle_{t}^{2}\right\rangle_{t^{\prime}}} \quad\{i=1,2\}
\end{aligned}
$$

with

$$
<A>_{t} \equiv \iiint \int d q_{1} d p_{1} d q_{2} d p_{2} A \rho(t) .
$$

In this case, the coordinate system $\left\{q_{1}, p_{1}, q_{2}, p_{2}\right\}$ looses its particular advantage in describing the bundle of trajectories under discussion. When there realizes a stationary state satisfying

$$
\frac{d}{d t}<q_{i}>_{t}=0, \quad \frac{d}{d t}\left\langle q_{i}^{2}-<q_{i}>_{t}^{2}\right\rangle_{t}=0, \quad \text { etc, }
$$

Equation (71) is considered to be a dynamical condition to characterize the system to be in the statistical object, because the system does not show any regularity associated with a certain specific coordinate system. In other words, the system has dynamically reached such a state that is coordinate system independent.

In Fig. 6, the time dependence of the variance $\left\langle p_{1}^{2}-\left\langle p_{1}\right\rangle^{2}\right\rangle$ of the momentum for the first degree of freedom is shown for the cases with $E=40$ and $V=-0.01,-0.04$ and -0.07 . A unit of time is given by $\tau_{1}=\omega_{1} / 2 \pi$, where $\omega_{1}$ is an eigen frequency of low-lying normal mode obtained by applying the RPA to Eq. (65). In the case with $V=-0.01$ where the whole phase space is covered by the regular motions illustrated in Fig. 1, the variance is oscillating and its amplitude is increasing. In the case with a much stronger interaction $V=-0.04$, the variance increases exponentially and then oscillates around some saturated value. Since the amplitude of oscillation is not small, a stationary state is not expected to be realized for a very long time. Note that the Poincaré section map for the case with $V=-0.04$ is still dominated by many kinds of island structure like the case with $V=-0.01$. Even though an initial distribution is chosen around the unstable fixed point where many trajectories with different characters 
come across with each other, domination of the KAM torus in the Poincaré section prevents the system from reaching some statistical object.

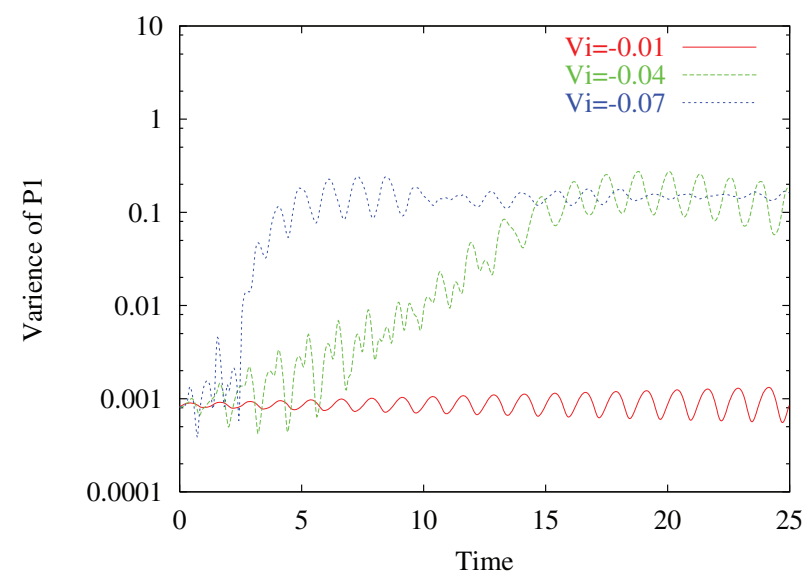

Fig. 6. Variance $\left\langle p_{1}^{2}-\left\langle p_{1}\right\rangle^{2}>\right.$ for the cases with $V=-0.01,-0.04$ and -0.07 .

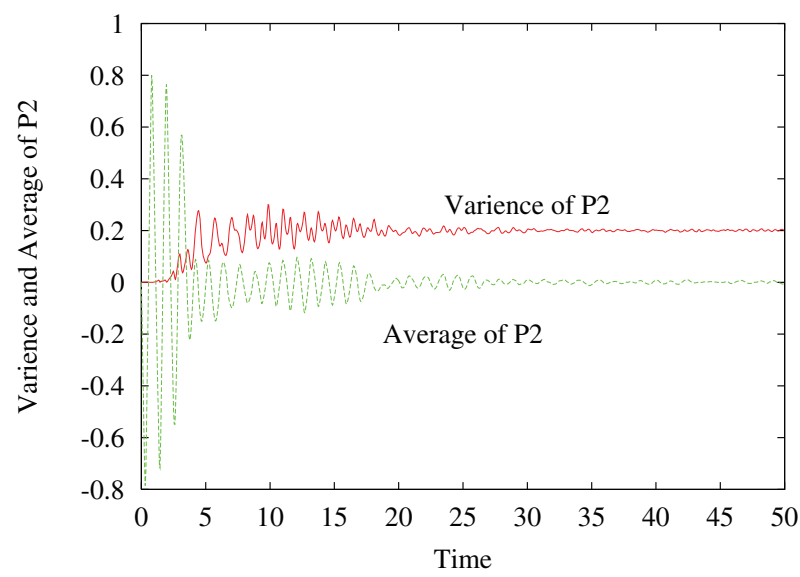

Fig. 7. Averaged value $<p_{2}>$ and variance $<p_{2}^{2}-<p_{2}>^{2}>$ for the case with $V=-0.07$.

In the case of $V=-0.07$, where the overwhelming region of the Poincare section map is covered by the chaotic sea as is depicted in Fig. 4, a quite different situation is realized. As is observed in Fig. 6, the time dependence of the variance of $p_{1}$ almost dies out around $\tau \approx 25 \tau_{1}$. In Fig. 7, the averaged value $\left\langle p_{2}\right\rangle$ and its variance $\left\langle p_{2}^{2}-\left\langle p_{2}\right\rangle^{2}\right\rangle$ for the second degree of freedom are shown for the case with $V=-0.07$. Since $\left\langle p_{2}\right\rangle$ has reached null value around $\tau \approx 30 \tau_{1}$ and the variance become almost constant around $\tau \approx 25 \tau_{1}$ like $<p_{1}^{2}-<$ $p_{1}>^{2}>$, the system is considered to be in a stationary statistical state where the relation in Eqs. (71) and (73) are well realized at around $\tau \approx 30 \tau_{1}$. In this case, the choice of a particular 
coordinate system does not have any profit for the present the system with $V=-0.07$, like the quantum system described by GOE.

Another information on the dynamic realization of the statistical state might be obtained from the two-time dynamic response $\chi_{l m}(t, \tau), X_{l m}(t, \tau)$ and correlation functions $\phi_{l m}(t, \tau)$, $\Phi_{l m}(t, \tau)$ defined in Eqs. (47) and (48). Suppose the system described by a bundle of trajectories has been developed exactly till $t_{I}$ following the original Liouville equation (28) or equivalently by the coupled-master equation (43). The approximate coupled master equations in Eqs. (49), which depend on the two-time dynamic response and correlation functions, are derived from Eq. (43) under the assumption that the effects coming from the fluctuation $H_{\Delta}(t)$ are sufficiently small so as to be evaluated by the second order perturbation theory. Consequently, Eqs. (49) is considered to be applicable either in a case with very small fluctuation effects or in a case with a very short time interval $\tau=t-t_{I}$ just after $t_{I}$. When one evaluates the $t_{I}$ dependence of the dynamic response and correlation functions

$$
\chi_{l m}\left(t_{I}+\tau, t_{I}\right), \quad \phi_{l m}\left(t_{I}+\tau, t_{I}\right), \quad X_{l m}\left(t_{I}+\tau, t_{I}\right), \quad \Phi_{l m}\left(t_{I}+\tau, t_{I}\right),
$$

one may study how their $\tau$-dependence change as a function of $t_{I}$. Since the dynamic response and correlation functions depend on the time derivative of $\left\langle q_{i}^{2}-\left\langle q_{i}\right\rangle_{t}^{2}\right\rangle$, their $t_{I}$-independence gives a more severe stationary condition than the condition (73).

When $\rho(t)$ reaches some stationary state after a long time-evolution through the original Liouville equation, the dynamic response and correlation functions show no $t_{I}$-dependence so as to be approximated by the usual one-time response and correlation functions appeared in the LRT. According to the recent work(22), it turned out that a dynamic realization of statistical state is established around $t_{I} \approx 50 \tau_{1}$ for the system described by the Hamiltonian in Eq. (66) with $V=-0.07$.

\subsubsection{Energy interchange between the collective and intrinsic systems}

Our attention is mainly focused on examining the energy interchange between these two systems, and what final states these two systems can reach and their interaction-dependence. For studying the energy interchange, we make numerical calculation for the following cases: The collective energy is much larger, comparable and much smaller than the intrinsic energy. Namely, the collective energy is chosen to be $E_{\eta}=20,40$ and 60, whereas the intrinsic energy is fixed at $E_{\xi}=40$. Here $E_{\xi}=40$ is chosen, because the phase space of the intrinsic system is almost covered by the chaotic sea at this energy. In order to examine the interaction dependence of the final state, the interaction strength parameter $\lambda$ is chosen to be 0.005 (relatively weak), 0.01 and 0.02 (relatively strong).

Figures 8 (a)-(d) show the time-dependent averaged values of the partial Hamiltonian $\left\langle H_{\eta}\right\rangle$, $\left\langle H_{\xi}\right\rangle$ and $\left\langle H_{\text {coupl }}\right\rangle$ and the total Hamiltonian $\langle H\rangle$ defined through

$$
\langle X\rangle=\int X \rho(t) d q d p \prod_{i=1}^{2} d q_{i} d p_{i},
$$

for the case with $E_{\eta}=40$. One may see that the main change occurs in the collective energy as well as the interaction energy, but not in the intrinsic energy.

When one precisely looks for the independent trajectories of the bundle, the collective, intrinsic and interaction energies of each trajectory are changing in time in accordance with the usual Hamilton system. Since the intrinsic system has already reached some stochastic 

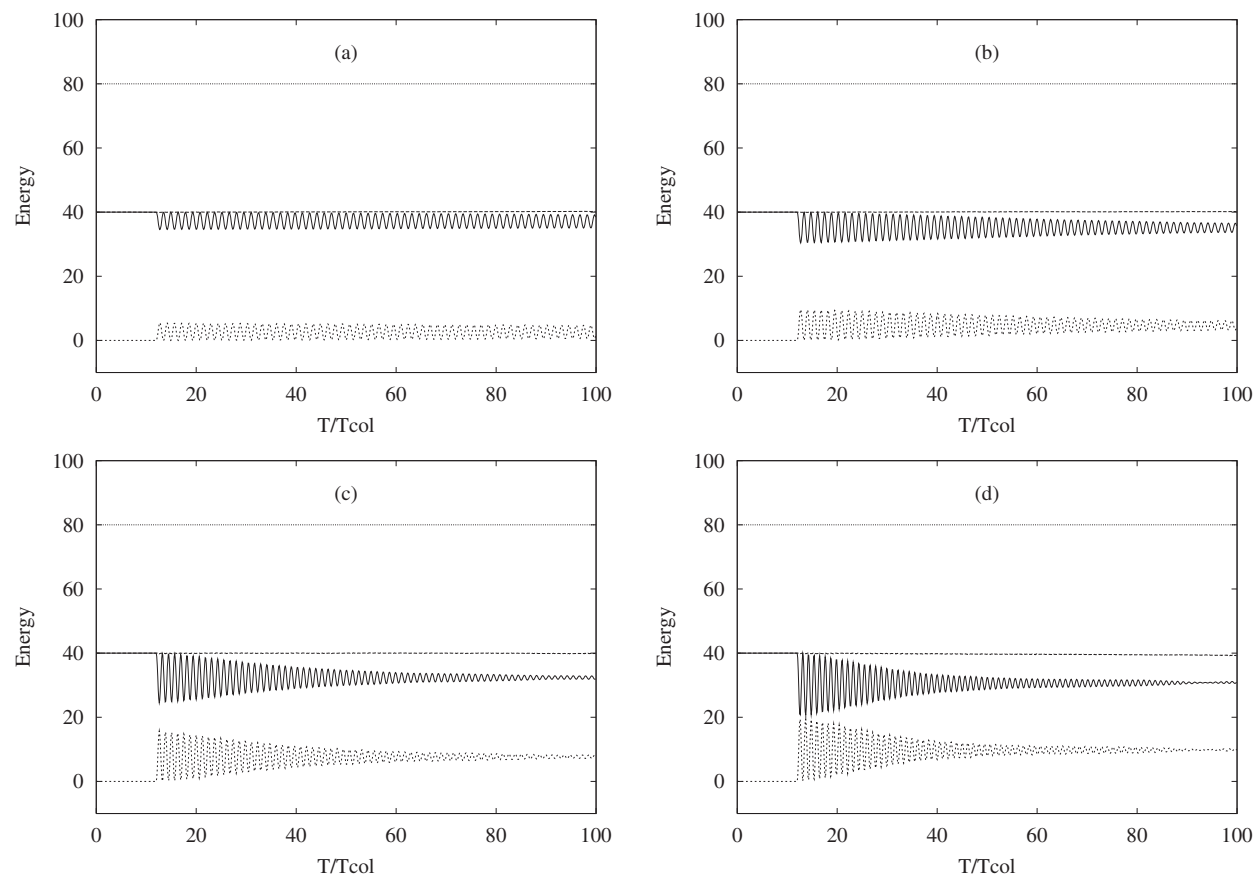

Fig. 8. Time-dependence of the averaged partial Hamiltonian $\left\langle H_{\eta}\right\rangle,\left\langle H_{\xi}\right\rangle,\left\langle H_{\text {coupl }}\right\rangle$ and $\langle H\rangle$ for $\mathrm{E}_{\eta}=40, \mathrm{E}_{\xi}=40$ and (a) $\lambda=0.005$; (b) $\lambda=0.01$; (c) $\lambda=0.02$ and (d) $\lambda=0.03$. Solid line refers to $\left\langle H_{\eta}\right\rangle$; long dashed line refers to $\left\langle H_{\xi}\right\rangle$; short dashed line refers to $\left\langle H_{\text {coupl }}\right\rangle$ and dotted line refers to $\langle H\rangle . \tau_{c o l}$ denotes a characteristic periodic time of collective oscillator.

state when the interaction is switch on, a time-dependence of the intrinsic energy for each trajectory is canceled out when one takes an average over many trajectories of the bundle. For a case with small interaction strength $(\lambda=0.005)$, the collective energy oscillates for a long time and seems not to reach any saturated value. In a case with a relatively large interaction strength $(\lambda \sim 0.02)$, it will reach some time-independent value.

Figures 9 (a) and (b) represent the numerical results for the cases with $E_{\eta}=20$ and 60, showing almost the same result as for the case with $E_{\eta}=40$.

From the above numerical simulation, one may see that the energy is dissipated from the collective to an 'environment', when the intrinsic system and the coupling interaction are regarded as an 'environment'. Before understanding the above energy transfer in terms of the phenomenological Langevin equation, it is important to microscopically explore what happens in the intrinsic system when the collective system is attached to the intrinsic system through the coupling interaction.

In Fig. 10, a time dependence of the variance of the intrinsic momentum $<p_{1}^{2}>$ is shown. The other intrinsic variances $\left\langle q_{1}^{2}\right\rangle,\left\langle q_{2}^{2}\right\rangle$ and $\left\langle p_{2}^{2}\right\rangle$ show almost the same time dependence as in Fig. 10. As discussed in our previous paper(22), an appearance of some chaotic state is expected when the variance has reached its stationary value. Since the variance of the intrinsic 

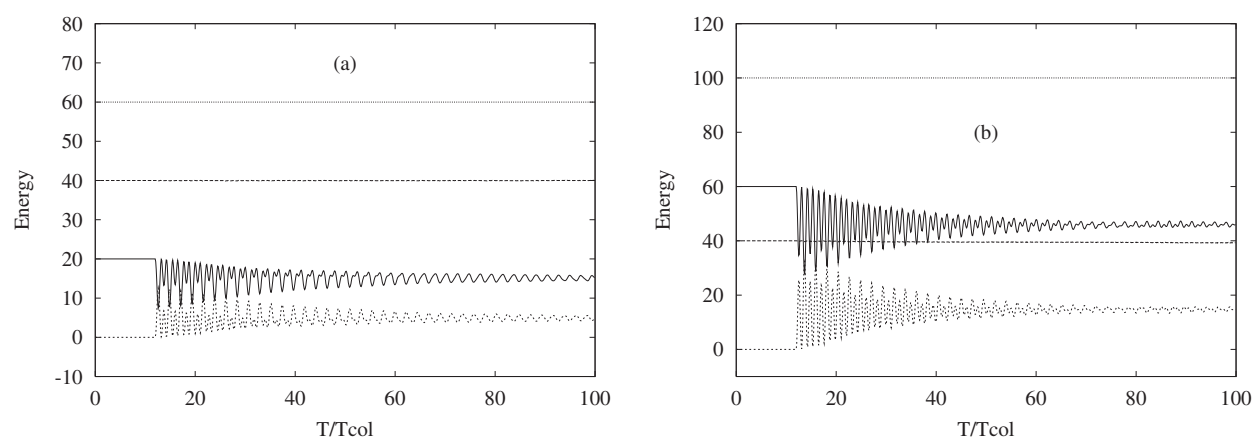

Fig. 9. Time-dependence of the averaged partial Hamiltonian for (a) $E_{\eta}=20, E_{\xi}=40, \lambda=0.02$; (b) $\mathrm{E}_{\eta}=60, \mathrm{E}_{\xi}=40, \lambda=0.02$. Reference of lines is the same as in Fig. 8.

system reaches some stationary value before $\tau_{s w}$ and since the intrinsic system is regarded to be in the chaotic state, the coupling interaction is activated at $\tau_{s w}$ in our simulation. After $\tau_{s w}=12 \tau_{c o l}$, its value remains almost the same for the small interaction strength case, and reaches quickly a little bit larger stationary value for the large coupling strength case $(\lambda=$ 0.02). This small increase corresponds to a slight enlargement of the chaotic sea in the intrinsic phase space. Practically, the values of variances are regarded to be constant before and after $\tau_{s w}$.

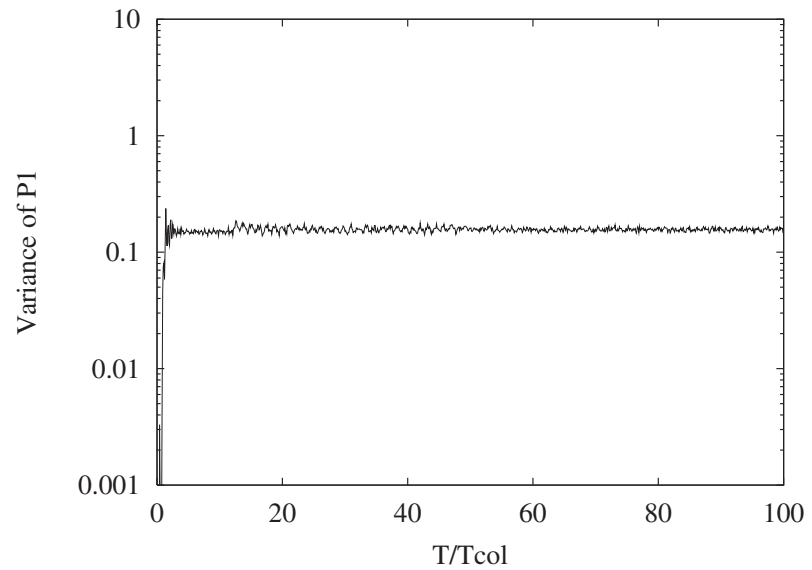

Fig. 10. Time-dependence of variance of $\mathrm{p}_{1}$ for $\mathrm{E}_{\eta}=40, \mathrm{E}_{\xi}=40$ and $\lambda=0.02$. Coupling is switch on at $\tau_{s w}=12 \tau_{c o l}$.

From our numerical simulation, one may deduce such a conclusion that the intrinsic system even with only two degrees of freedom can be treated as a time independent statistical object before and after the coupling interaction is activated. This conclusion provide us with the dynamical foundation for understanding the statistical ansatz adopted in the conventional 
transport theory, where the irrelevant system is always regarded as a time-independent statistical object.

Since the variance has reached its stationary value shortly after $\tau_{s w}$, it is reasonable to introduce the following time independent quantity:

$$
<p_{i}^{2}+q_{i}^{2}>=\int \prod_{i=1}^{2} d p_{i} d q_{i}\left\{p_{i}^{2}+q_{i}^{2}\right\} \rho(t)
$$

In accordance with the mean-field Liouvillian in Eq. (44), one may introduce the time-independent collective mean-field Hamiltonian as

$$
H_{\eta}+\left.H_{\eta}(t)\right|_{t>\tau_{s w}}=\frac{p^{2}}{2 M}+\frac{1}{2} M \omega_{0}^{2} q^{2}+\lambda\left(q-q_{0}\right)^{2} \sum_{i=1}^{2}<p_{i}^{2}+q_{i}^{2}>.
$$

Except for the effects coming from the fluctuation part $H_{\Delta}(t)$, the collective trajectory is supposed to be described by the mean field Hamiltonian in Eq. (77) after the coupling interaction is switch on. The solution of Eq. (77) is expressed as

$$
q=A \cos \omega\left(t-\tau_{s w}\right), \quad p=-M \omega A \sin \omega\left(t-\tau_{s w}\right),
$$

where

$$
\omega^{2}=\omega_{0}^{2}+\omega_{1}^{2}, \quad \omega_{1}^{2} \equiv \frac{2 \lambda}{M}<p_{i}^{2}+q_{i}^{2}>, \quad A=q_{0}\left(\frac{\omega_{0}}{\omega}\right)^{2},
$$

the amplitude $A$ being fixed by using the initial condition $q\left(\tau_{s w}\right)=q_{0}$. In accordance with this initial condition, there holds the following energy conservation before and after $\tau_{s w}$ as

$$
\left.H_{\eta}\right|_{t=\tau_{s w}-0}=H_{\eta}+\left.H_{\eta}(t)\right|_{t=\tau_{s w}+0}=\frac{M}{2} q_{0}^{2} \omega_{0}^{2} .
$$

In order to understand a oscillating property of the collective energy observed in Figs. 1 and 2 , let us substitute the solution in Eq. (78) into the collective Hamiltonian $H_{\eta}$. Then one gets

$$
H_{\eta}=\frac{M}{2} q_{0}^{2} \omega_{0}^{2}\left\{1-4 \frac{\omega_{1}^{2} \omega_{0}^{2}}{\omega^{4}} \sin ^{4} \frac{\omega}{2}\left(t-\tau_{s w}\right)\right\} .
$$

In Fig. 11, the numerical result of Eq. (81) is shown together with the exact simulated result. As is clearly recognized from Fig. 11 and Eq. (81), the mean field description can well reproduce the oscillating property (the amplitude, the central energy of the oscillation as well as the frequency) of the collective energy $\left\langle H_{\eta}\right\rangle$, whereas it can not reproduce a reduction mechanism of the amplitude. That is the mean field Hamiltonian can not describe the dissipation process. More precisely, one may see that the mean-field approximation provides us with a decisive information on the following two points: (a) the amplitude $A$ of the collective energy is mainly determined by the coupling interaction strength $\lambda$ as well as the averaged properties of the intrinsic system $\left\langle\sum_{i=1}^{2} p_{i}^{2}+q_{i}^{2}\right\rangle ;(\mathrm{b})$ the frequency $\omega$ is related with the characteristic frequency of the collective oscillator $\omega_{0}$, the coupling interaction strength $\lambda$ and the averaged properties of intrinsic system $\left\langle\sum_{i=1}^{2} p_{i}^{2}+q_{i}^{2}\right\rangle$. From the above discussion 


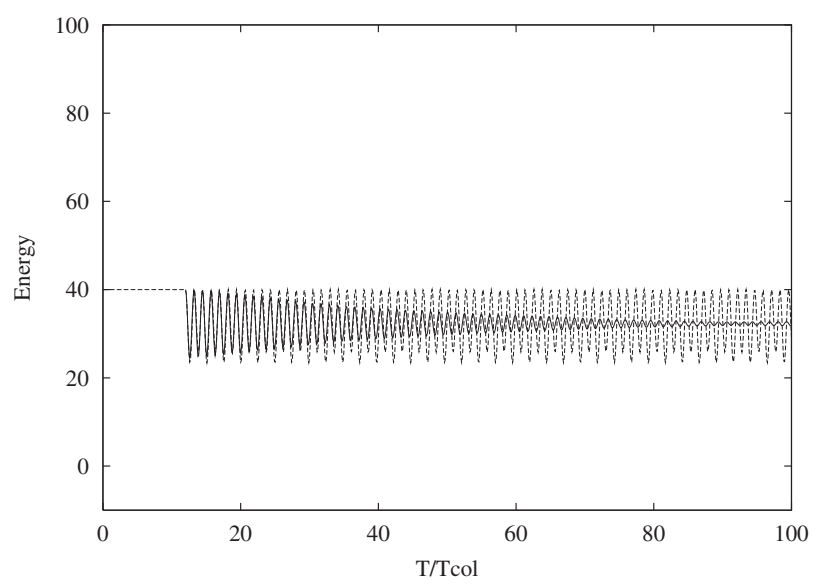

Fig. 11. Time-dependence of average collective energy (dashed line) $H_{\eta}$ in Eq. (81), in which the mean-field energy of the coupling interaction is considered as shown in Eq. (77), together with the exact simulated result (solid line). Parameters used in the mean-field potential is the same as Fig. 8(c).

and from Figs.1 and 2, the dissipation process should be attributed to the fluctuation effects coming from $H_{\Delta}$.

\subsubsection{Analysis with a phenomenological transport equation}

Before discussing the microscopic dynamics responsible for the damping and diffusion process, let us apply the phenomenological transport equation to our present simulated process. Let us suppose that the collective motion will be subject to both a friction force and a random force, and can be described by the Langevin equation. A simple Langevin equation is given by

$$
M \ddot{q}+\frac{\partial U^{m f}(q)}{\partial q}+\gamma \dot{q}=f(t),
$$

where $U^{m f}(q)$ represents the potential part of $H_{\eta}+H_{\eta}(t)$ in Eq. (77) and $\gamma$ the friction strength parameter. A function $f(t)$ represents the random force and, in our calculation, it is taken to be the Gaussian white noise characterized by the following moments:

$$
\langle f(t)\rangle=0, \quad\langle f(t) f(s)\rangle=k T \delta(t-s) .
$$

The numerical result for Eq. (82) is shown in Fig. 12 with the parameters $\gamma=0.0033$ and $k T=1.45$. The used parameters appearing in $U^{m f}(q)$ is the same as in Fig. 8 (c).

As is understood from Fig. 12, the Langevin equation do reproduce the energy transfer from the collective system to the environment quite well. This means that our dynamical simulation shown in Fig. 8 is satisfactory linked with the conventional transport equation, and our schematic model Hamiltonian introduced by Eqs. (64), (66) and (67) is successfully considered as a dynamical analogue of the Brownian particle coupled with the classical statistical system. Based on the above analogy and on Eqs. (57) and (82), one may learn the collective degree of freedom is subject to both an average force coming from the mean field Hamiltonian in 


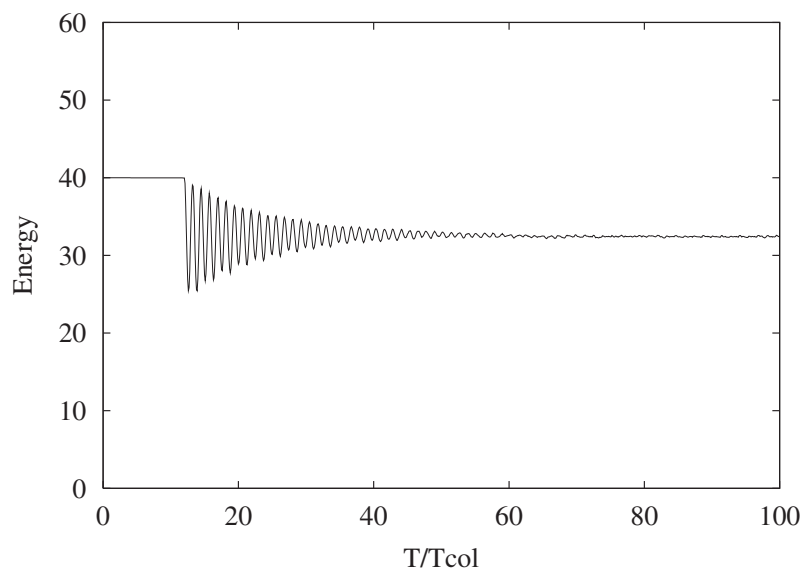

Fig. 12. Time-dependence of average collective energy simulated with Langevin equation (82) with $\gamma=0.0033$ and $\mathrm{kT}=1.45$. Parameters used in the mean-field potential is the same as Fig. 8(c).

Eq. (77) and the fluctuation term $H_{\Delta}$. Namely, the fluctuation $H_{\Delta}$ described by the last three terms on the right hand side of Eq. (57) is responsible for not only the damping of the oscillation amplitude but also for the dissipative energy flow from the collective system to the environment.

At the end of this subsection, it should be noticed that our choice of $\gamma$ and $k T$ does not satisfy the fluctuation-dissipation theorem. This means that our simulated dissipative phenomenon is not the same as the usual damping phenomena described within the LRT. Since our simulated dissipation phenomenon is induced not by the linear coupling but by the nonlinear coupling, there still remain interesting questions for comprehensively understanding the macroscopic transport phenomena.

\subsubsection{Microscopic origin of damping and diffusion mechanism}

In the Langevin equation, there are two important forces, the friction force and the random force. The former describes the average effect on the collective degree of freedom causing an irreversible dissipation, while the latter the diffusion of it. According to the parameter values adopted in our Langevin simulation in Fig. 12, it is naturally expected that the dissipative-diffusion mechanism plays a crucial role in reducing the oscillation amplitude of collective energy, and in realizing the steadily energy flow from the collective system to the environment.

In order to explore this point, a time development of the collective distribution function $\rho_{\eta}(t)$ is shown in Figs. 13 and 14 for two cases with $\lambda=0.005$ (small coupling strength) and 0.02 (large coupling strength), respectively. In Figs. 13(a) and 14(a), it is illustrated how a shape of the distribution function $\rho_{\eta}(t)$ in the collective phase space disperses depending on time. In these figures, an effect of the friction force ought to be observed when a location of the distribution function changes from the outside (higher energy) region to the inside (lower energy) region of the phase space. On the other hand, a dissipative diffusion mechanism is 

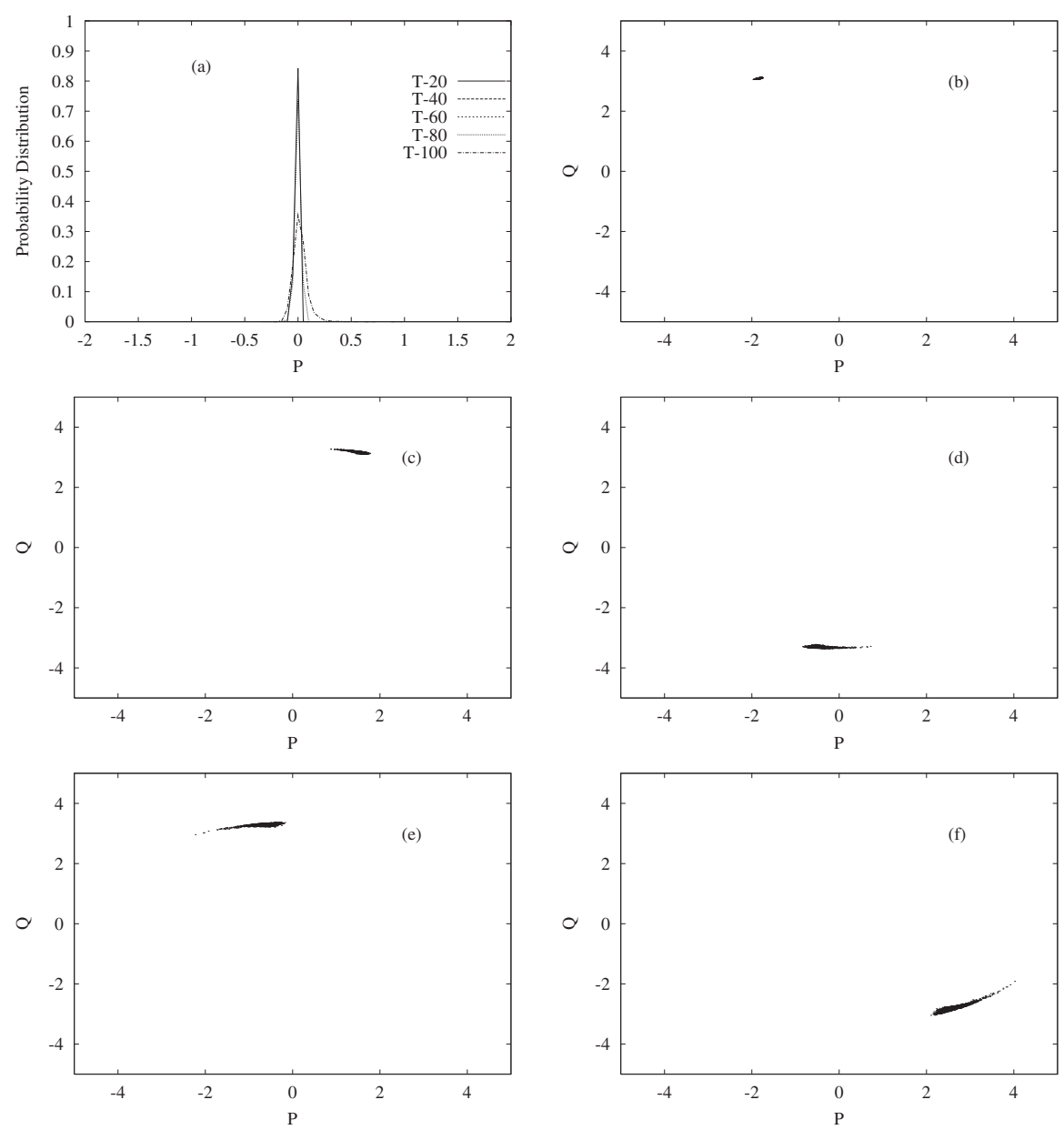

Fig. 13. (a) Probability distribution function of collective trajectories which is defined as $P D_{\eta}\left(p^{\prime}\right)=\left.\int \rho_{\eta}(t)\right|_{p=p_{m}+p^{\prime}} d q$ and $p_{m}$ satisfies $\left.\frac{\partial \rho_{\eta}(t)}{\partial p}\right|_{p=p_{m}}=0 ;(\mathrm{b}-\mathrm{f})$ the collective distribution function in (p,q) space at $\mathrm{T}=20 \tau_{c o l} ; \mathrm{T}=40 \tau_{c o l} ; \mathrm{T}=60 \tau_{c o l} ; \mathrm{T}=80 \tau_{c o l} ;$ and $\mathrm{T}=100 \tau_{c o l}$ for $\mathrm{E}_{\eta}=40, \lambda=0.005$. The parameters are the same as in Fig. 8(c).

studied from Figs. 13(a) and 14(a) by observing how strongly a distribution function initially (at $t=\tau_{s w}$ ) centered at one point in the collective phase space disperses depending on time. One may see that for the case with $\lambda=0.005, \rho_{\eta}(t)$ is slightly enlarged from the initial $\delta$-distribution, but is still concentrated in a rather small region even at $t=100 \tau_{\text {col }}$. On the other hand, for the case with $\lambda=0.02$, one may see that $\rho_{\eta}(t)$ quickly disperses after the coupling interaction is switch on and tends to cover a whole ring shape in the phase space at $t=100 \tau_{\text {col }}$. 

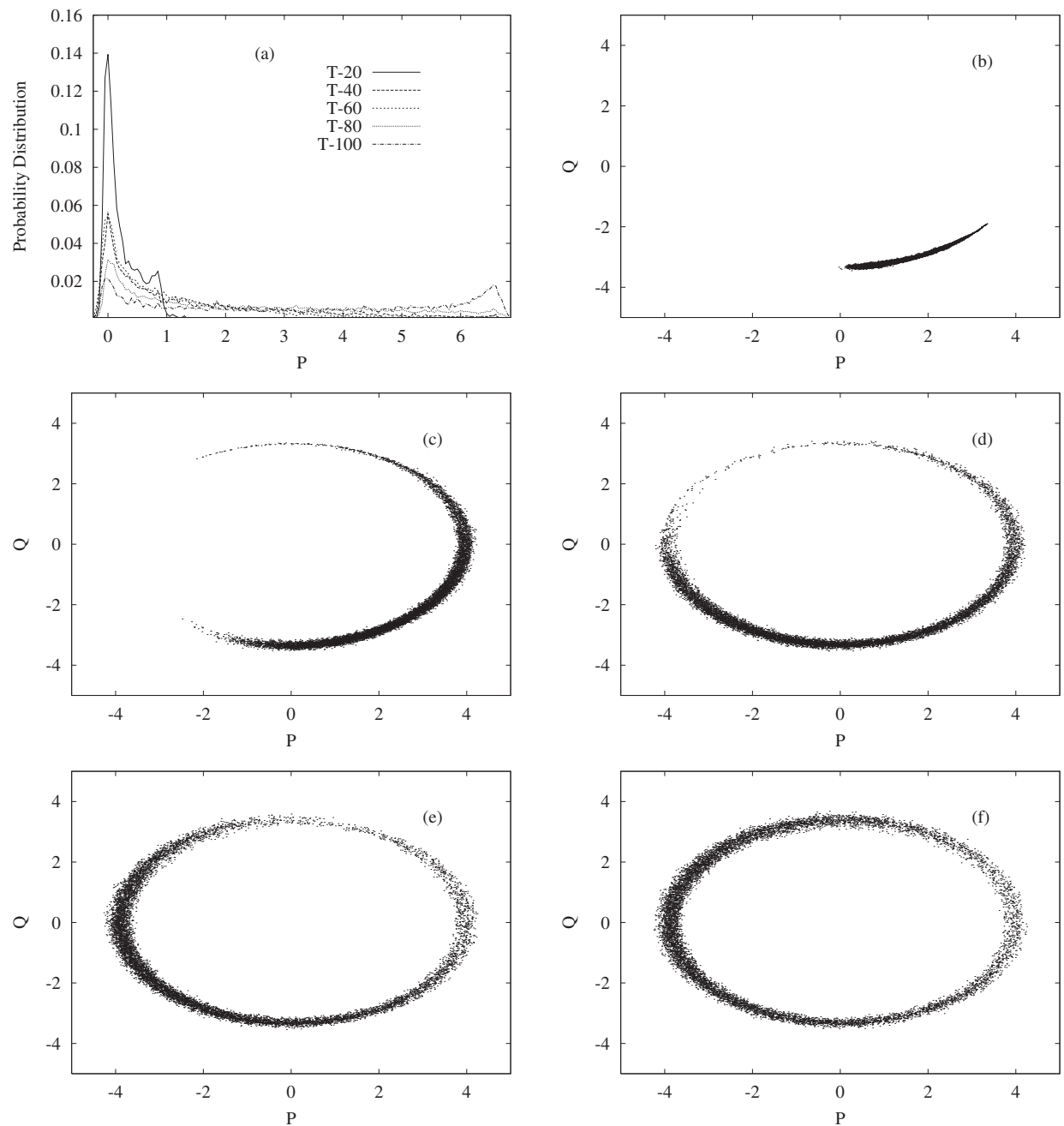

Fig. 14. (a) Probability distribution function of collective trajectories which is defined as $P D_{\eta}\left(p^{\prime}\right)=\left.\int \rho_{\eta}(t)\right|_{p=p_{m}+p^{\prime}} d q$ and $p_{m}$ satisfies $\left.\frac{\partial \rho_{\eta}(t)}{\partial p}\right|_{p=p_{m}}=0 ;(\mathrm{b}-\mathrm{f})$ the collective distribution function in (p,q) space at $\mathrm{T}=20 \tau_{c o l} ; \mathrm{T}=40 \tau_{c o l} ; \mathrm{T}=60 \tau_{c o l} ; \mathrm{T}=80 \tau_{c o l} ;$ and $\mathrm{T}=100 \tau_{c o l}$ for $\mathrm{E}_{\eta}=40, \lambda=0.02$. The parameters are the same as in Fig. 8(c).

Let us discuss a relation between the reduction mechanism in the amplitude of collective energy and the dispersing property of $\rho_{\eta}(t)$. Suppose $\rho_{\eta}(t)$ does not show any strong disperse property by almost keeping its original $\delta$-function shape, in this case, the effects coming from $H_{\Delta}(t)$ is considered to be small. The collective part of each trajectory has a time dependence expressed in Eq. (78) and its collective energy $H_{\eta}$ has a time dependence given by Eq. (81). 

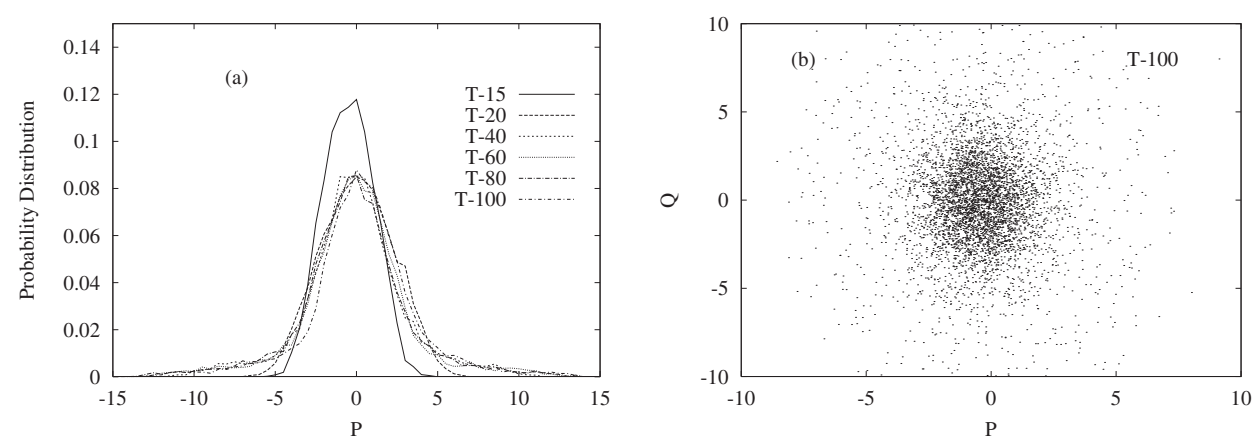

Fig. 15. (a) The probability distribution function of collective trajectories as defined in the caption of Fig. 13(a); (b) collective distribution function in (p,q) space at $\mathrm{t}=100 \tau_{\text {col }}$ simulated with Langevin equation (82) with $\gamma=0.0033$ and $\mathrm{kT}=1.45$. The parameters used in mean-field potential is the same as Fig. 8(c)

Since there is a well developed coherence among the trajectories in $\rho_{\eta}(t)$ when $\lambda=0.005$, the averaged collective energy $\left\langle H_{\eta}>\right.$ over the bundle of trajectories still has a time dependence given by Eq. (81). Consequently, one may not expect a reduction of the oscillation amplitude in the collective energy as is shown in Fig. 8(a).

When the distribution function tends to expand over the whole ring shape, the collective part of each trajectory is not expected to have the same time dependence as in Eq. (78). This is due to the effects coming from the stochastic force $H_{\Delta}(t)$, and some trajectories have a chance to have an advanced phase whereas other trajectories have a retarded phase in comparison with the phase in Eq. (78). According to the decoherent effects coming from $H_{\Delta}(t)$, the time dependence of the collective energy for the each trajectory in Eq. (78) cancels out due to the randomness of the phases when one takes an average over the bundle of trajectories. This dephasing mechanism is induced by $H_{\Delta}(t)$, and is considered to be the microscopic origin of the damping, i.e. the energy transfer from the collective system to the environment.

In order to compare the above mechanism with what happens in the phenomenological transport equation, the solution of the Langevin equation represented in the collective phase space is shown in Fig. 15 for the cases with $\gamma=0.0033$ and $k T=1.45$. From this figure, one may understand that the damping (a change of the distribution from the outside to the inside of the phase space) as well as the diffusion (an expansion of the distribution) are taking place so as to reproduce the numerical result in Fig. 12. Even though the Langevin equation gives almost the same result as in Fig.8 in the macroscopic-level, as is recognized by comparing Figs. 13 and 14 with Fig. 15, there are substantial differences in the microscopic-level dynamics. Namely, the distribution function $\rho_{\eta}(t)$ of our simulation evolves into the whole ring shape with staying almost the same initial energy region of the phase space, while the solution of the Langevin equation evolves to a round shape with covering the whole energetically allowed region. In the case of the Langevin simulation, the dissipation and dephasing mechanisms are seemed to contribute to reproduce the result in Fig. 12, while the dephasing mechanism is essential for the damping of the collective energy in our microscopic simulation.

Here, it is worthwhile mentioning that the decoherence or dephasing process due to the interaction with the environment has also been discussed in the quantum system(13; 73). 


\subsection{The Case of the system with multi-degree of freedom}

As shown in last section, it has been clarified that the main damping mechanism(30) of the collective motion nonlinearly coupled with the intrinsic system composed by two degrees of freedom is dephasing caused by the chaoticity of intrinsic system. Here, it should be noted that the dephasing process only appears under the nonlinear coupling interaction, specially for the small number of degrees of freedom, as in a case of the quantum dynamical system(13). It was also found that the collective distribution function organized by the Liouville equation and that by the phenomenological Langevin equation show quite different structure in the collective phase space, even though they give almost the same macro-level description for the averaged property of the collective motion.

Now we are facing the questions as how to understand such the difference between two descriptions, and in what condition we can expect the same microscopic situation as the Langevin equation described and when the fluctuation-dissipation theorem comes true. In fact, underlying the conventional approach to the Fokker-Planck- or Langevin-type equation, the intrinsic subsystem is considered with large (or say, in $\square$ nite) number of degrees of freedom placed in an initial state of canonical equilibrium. So, for understanding the fundamental background of phenomenological transport equation and the basis of dissipation-dissipation relation, there still remain interesting questions for comprehensively understanding the effect, which changes depending on the number of degrees of freedom of intrinsic system.

For this purpose, we will use a Fermi-Pasta-Ulam (FPU) system for describing the intrinsic system, which allows us more conveniently to change the number of degrees of freedom of intrinsic system. It will be shown that dephasing mechanism is the main mechanism for small number degrees of freedom (say, two) case. When the number of degrees of freedom becomes relative large (say, eight or more), the diffusion mechanism will start to play the role and the energy transport process can be divided into three regimes, such as a dephasing regime, a statistical relaxation regime, and an equilibrium regime. By examining the time evolution of entropy with using the nonextensive thermodynamics in Sec. 4, we will find that an existence of three regimes is clearly shown.

Under the help of analytical analysis carried in Sec. 5, we will also show that for the case with relative large number of degrees of freedom, the energy transport process can be described by the generalized Fokker-Planck- and Langevin-type equation, and a phenomenological Fluctuation-Dissipation relation is satisfied. For the finite system, the intrinsic system plays the role as a finite heat bath with finite correlation time and the statistical relaxation is anomalous diffusion. Only for the intrinsic system with very large number of degrees of freedom, the dynamical description and conventional transport approach may provide almost the same macro- and micro-level mechanisms.

\subsection{1 $\beta$-fermi-pasta-Ulam (FPU) system}

The collective subsystem, for simplicity and without any lose of generality, is represented by a harmonic oscillator as the case of the system with three degrees of freedom as Eq. (64). The intrinsic subsystem, mimicking the environment, is described by a $\beta$ Fermi-Pasta-Ulam (FPU) system (sometime called $\beta$-FPU system, as with quadrtic interaction), which was posed in the 
famous paper (49) and reviewed in (74):

$$
\begin{aligned}
& H_{\xi}=\sum_{i=1}^{N_{d}} \frac{p_{i}^{2}}{2}+\sum_{i=2}^{N_{d}} W\left(q_{i}-q_{i-1}\right)+W\left(q_{N_{d}}\right), \\
& W(q)=\frac{q^{4}}{4}+\frac{q^{2}}{2}
\end{aligned}
$$

where

$$
\begin{aligned}
& q=\frac{1}{\sqrt{2}}\left(\eta+\eta^{*}\right), \quad p=\frac{i}{\sqrt{2}}\left(\eta^{*}-\eta\right), \\
& q_{i}=\frac{1}{\sqrt{2}}\left(\xi_{i}+\xi_{i}^{*}\right), \quad p_{i}=\frac{i}{\sqrt{2}}\left(\xi_{i}^{*}-\xi_{i}\right), \quad\left\{i=1, \cdots, N_{d}\right\}
\end{aligned}
$$

$N_{d}$ represents the number of degrees of freedom (i.e., the number of nonlinear oscillators). According to the related literatures $(26 ; 48 ; 74)$, the dynamics of $\beta$-FPU becomes strongly chaotic and relaxation is fast, when the energy per DOF $\epsilon$ is chosen to be larger than a certain value (called as the critical value(48), say $\epsilon_{c} \approx 0.1$ ). In the this thesis, $\epsilon$ is chosen as 10 to guarantee that our irrelevant subsystem can reach fully chaotic situation. Indeed, in this case, the calculated largest Lyapunov exponent $\sigma\left(N_{d}\right)$ turns out to be positive, for instance $\sigma\left(N_{d}\right)=0.15,0.11$, and 0.11 for $N_{d}=2,4$, and 8, respectively. Thus, a "fully developed chaos" is expected for the $\beta$-FPU system, and an appearance of statistical behavior in its chain of oscillators and an energy equipartition among the modes are expected to be realized.

For the coupling interaction, we use the following nonlinear interaction given by

$$
H_{\text {coupl }}=\lambda\left\{q^{2}-q_{0}^{2}\right\}\left\{q_{1}^{2}-q_{1,0}^{2}\right\} .
$$

A physical meaning of introducing the quantities $q_{0}$ and $q_{1,0}$ in Eq. (86) is discussed in Sec. 3.1.1 and our previous paper(30). Such the choice of the coupling interaction form means that $q_{1}$ is considered as a doorway variable, through which the intrinsic subsystem exerts its influence on the collective subsystem(26). It should be pointed out that the form of coupling interaction in Eq. (86) is a little bit different from the one used in our previous paper(30) since here we want to treat the collective and doorway variables in a more parallel way. The numerical comparison between this two forms shows there is no substantial differences between these two forms on the final results.

As discussed in Sec. 3.1.1, the time evolution of the distribution function $\rho(t)$ is evaluated by using the pseudo-particle method as:

$$
\begin{array}{r}
\rho(t)=\frac{1}{N_{p}} \sum_{n=1}^{N_{p}} \prod_{i=1}^{N_{d}} \delta\left(q_{i}-q_{i, n}(t)\right) \delta\left(p_{i}-p_{i, n}(t)\right) \\
\cdot \delta\left(q-q_{n}(t)\right) \delta\left(p-p_{n}(t)\right),
\end{array}
$$

where $N_{p}$ means the total number of pseudo-particles. The distribution function in Eq. (87) defines an ensemble of the system, each member of which is composed of a collective degree of freedom coupled to a single intrinsic trajectory. The collective coordinates $q_{n}(t)$ and $p_{n}(t)$, and the intrinsic coordinates $q_{i, n}(t)$ and $p_{i, n}(t)\left\{i=1, \cdots, N_{d}\right\}$ determine a phase space point 
of the $n$-th pseudo-particle at time $\mathrm{t}$, whose time dependence is described by the canonical equations of motion given by

$$
\begin{aligned}
& \dot{q}_{i}=\frac{\partial H}{\partial p_{i}}, \quad \dot{p}_{i}=-\frac{\partial H}{\partial q_{i}}, \quad\left\{i=1, \cdots, N_{d}\right\} \\
& \dot{q}=\frac{\partial H}{\partial p}, \quad \dot{p}=-\frac{\partial H}{\partial q}
\end{aligned}
$$

We use the fourth order simplectic Runge-Kutta algorithm(75; 76) for integrating the canonical equations of motion and $N_{p}$ is chosen to be 10,000. In our study, the coupling strength parameter is chosen as $\lambda \sim 0.002$.

\subsubsection{Energy dissipation and equipartition}

We have discussed a microscopic dynamical system with three degrees of freedom in Sec. 3.1.3, 3.1.4 and 3.1.5, and shown that the dephasing mechanism induced by fluctuation mechanism turned out to be responsible for the energy transfer from collective subsystem to environment(30). In that case, as we shown, the fluctuation-dissipation relation does not hold and there is substantial difference in the microscopic behaviors between the microscopic dynamical simulation based on the Liouville equation and the phenomenological transport equation even if these two descriptions provide almost same macroscopic behaviors. Namely, the collective distribution function organized by the Liouville equation evolves into the whole ring shape with staying almost the same initial energy region of the phase space, while the solution of the Langevin equation evolves to a round shape, whose collective energy is ranging from the initial value to zero.

For answering the questions as how to understand the above stated differences, and in what condition where the microscopic descriptions by the Langevin equation and by the Liouville equation give the same results and in what physical situation where the fluctuation-dissipation theorem comes true, a naturally extension of our work(30) is to considered the effects of the number of degrees of freedom in intrinsic subsystem because no matter how our simulated dissipation phenomenon is obtained by a simplest system which is composed of only three degrees of freedom, as described in Sec. 3.1.

In our numerical calculation, the used parameters are $M=1, \omega^{2}=0.2$. In this case, the collective time scale $\tau_{c o l}$ characterized by the harmonic oscillator in Eq. (64) and the intrinsic time scale $\tau_{\text {in }}$ characterized by the harmonic part of the intrinsic Hamiltonian in Eq.(84) satisfies a relation $\tau_{c o l} \gg \tau_{i n}$. The switch-on time $\tau_{s w}$ is set to be $\tau_{s w}=100 \tau_{c o l}$

Figures $16(\mathrm{a}-\mathrm{d})$ show the time-dependent averaged values of the partial Hamiltonian $\left\langle H_{\eta}\right\rangle$, $\left\langle H_{\xi}\right\rangle$ and $\left\langle H_{\text {coupl }}\right\rangle$ and the total Hamiltonian $\langle H\rangle$ for the case with $E_{\eta}=30, \lambda=0.002, N_{d}=2,4$, 8 and 16, respectively. The definition of ensemble average is the same as Eq. (75).

In order to show how the dissipation of the collective energy changes depending on the number of degrees of freedom in intrinsic subsystem, the time-dependent averaged values of the partial Hamiltonian $\left\langle H_{\eta}\right\rangle$ are also shown in Fig. 17 for the cases with $N_{d}=2,4,8$ and 16 , respectively.

It can be clearly seen that a very similar result has been obtained for the case with $N_{d}=2$ as described in our previous paper(30), that is, the main change occurs in the collective energy as well as the interaction energy, and the main process responsible for this change is coming from the dephasing mechanism. One may also learn from our previous paper(30) that the dissipative-diffusion mechanism plays a crucial role in reducing the oscillation amplitude of 

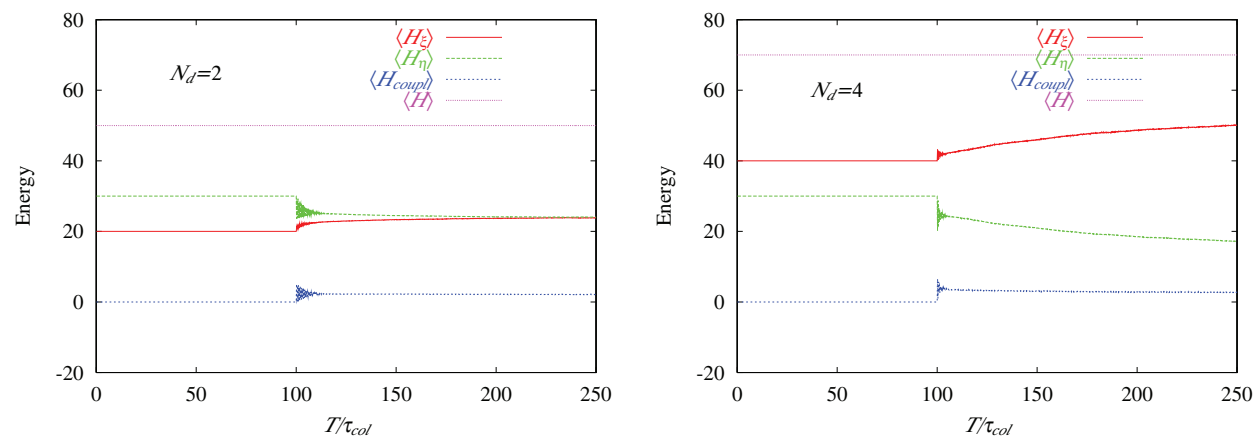

Fig. 16. Time-dependence of the average partial Hamiltonian $\left\langle H_{\eta}\right\rangle,\left\langle H_{\xi}\right\rangle,\left\langle H_{\text {coupl }}\right\rangle$ and the total Hamiltonian $\langle H\rangle$ for $\mathrm{E}_{\eta}=30, \lambda=0.002$. (a) $N_{d}=2$, (b) $N_{d}=4$, (c) $N_{d}=8$ and (d) $N_{d}=16$.
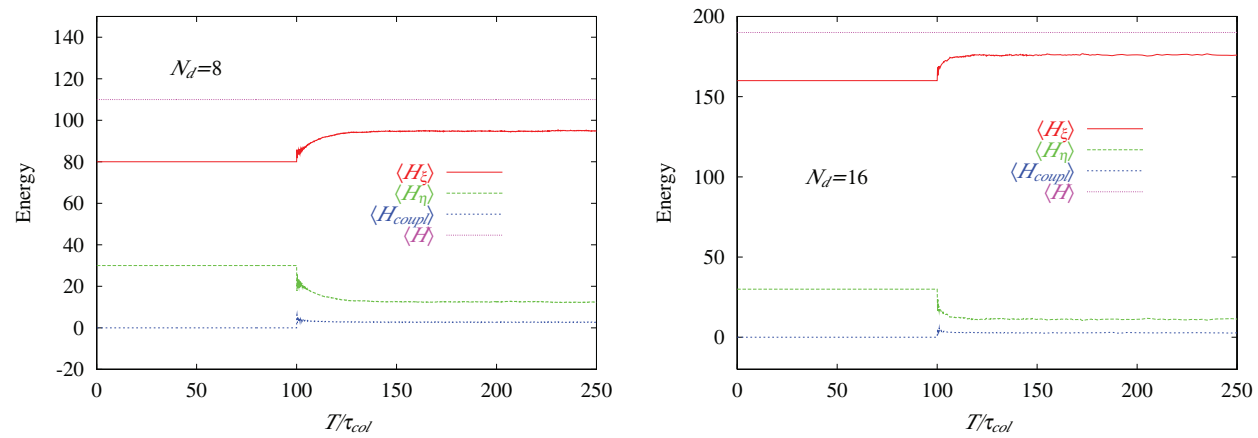

Fig. 16. continued.

\begin{tabular}{|c|cccc|}
\hline$N_{d}$ & 2 & 4 & 8 & 16 \\
\hline$\left\langle H_{\xi}\right\rangle$ & 11.92 & 12.54 & 11.851 & 10.996 \\
$\left\langle H_{\eta}\right\rangle$ & 24.03 & 17.15 & 12.499 & 11.32 \\
\hline
\end{tabular}

Table 1. An asymptotic average energy for every degree of freedom in the intrinsic system and that for the collective system

collective energy, and in realizing the steadily energy flow from the collective system to the environment.

However, with the increasing of the number of degrees of freedom of intrinsic subsystem, the collective energy, after finishing the dephasing process, gradually decreases and finally reaches to a saturated value. This saturated asymptotic may be understood as a realization of the dynamics balance between an input of energy into the collective subsystem from the fluctuation of nonlinear coupling interaction between the two subsystems and an output of energy due to its dissipation into the environment. It is no doubt that there appears another mechanism for the $N_{d}$ larger than 2 . 


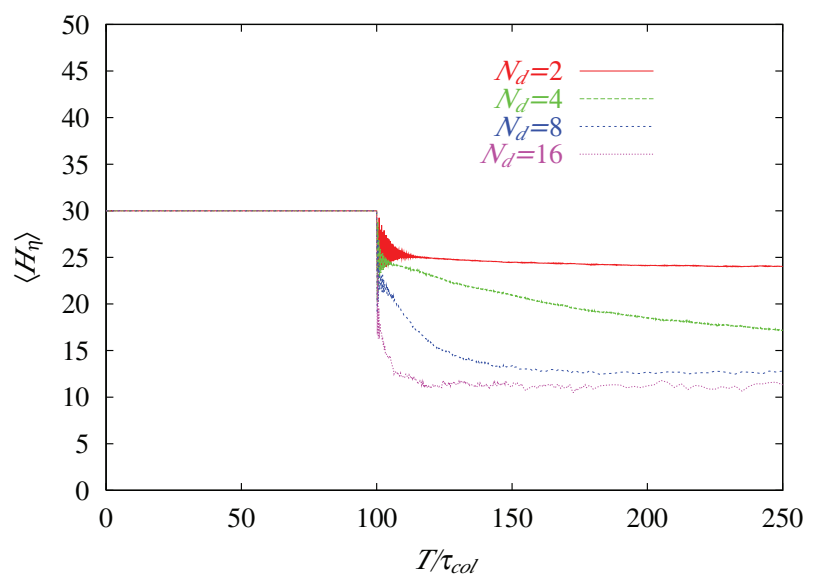

Fig. 17. Time-dependent average value of collective energy $\left\langle H_{\text {col }}\right\rangle$ for the cases with $N_{d}=2,4$, 8 and 16. Parameters are the same as Fig. 16.

Here we also should noticed the asymptotic average energies for every degrees of freedom in the intrinsic subsystem and that of collective subsystem as shown in Table 1. Considering a boundary effect of the finite system. i.e., the two ends oscillator in $\beta$-FPU Hamiltonian, one may see that the equipartition of the energy among every degree of freedom is expected in the final stage for the case with relatively large number of degrees of freedom, as $N_{d} \geq 8$.

\subsubsection{Three regimes of collective dissipation dynamics}

One can understand from Figs. 16 and 17 that the energy transfer process of collective subsystem can be divided into three regimes: (1) Dephasing regime. In this regime, the fluctuation interaction reduces the coherence of collective trajectories and damps the average amplitude of collective motion. This regime is the main process in the case when system with small number of degrees of freedom (say, two). When the number of degrees of freedom increases, the time scale of this regime will decrease; (2) Non-equilibrium relaxation regime, which will also be called as thermodynamical regime in the next section. In this regime, the energy of collective motion irreversibly transfers to the "environment"; (3) Saturation regime. This is an asymptotic regime where the total system reaches to another equilibrium situation and the total energy is equally distributed over every degree of freedom realized in the cases with $N_{d} \geq 8$. We will mention above three regimes again in our further discussion.

From the conventional viewpoint of transport theory, we can see that such the gradually decreasing behaviour of collective energy is due to an irreversible dissipative perturbation, which comes from the interaction with intrinsic subsystem and damps the collective motion. The asymptotic and saturated behaviour reveals that a fluctuation-dissipation relation may be expected for the cases with $N_{d} \geq 8$. Remembering our previous simulation(30) by using Langevin equation for the case with $N_{d}=2$, we can see, in that case, the role of fluctuation interaction mainly contribute to provide the diffusion effect which reduce the coherence of collective trajectories. The irreversible dissipative perturbation (friction force) is relatively small. However, an appearance of the second regime may indicate that the contribution of the dissipative (damping) mechanism will become large with the increasing of $N_{d}$. We will show 
in the next section that it is the dissipative (damping) mechanism that makes the collective distribution function of the cases with $N_{d} \geq 8$ evolves to cover the whole energetically allowed region as the solution of the Langevin equation. In this sense, one may expect that the above mentioned numerical simulation provide us with very richer information about the dissipative behaviour of collective subsystem, which changes depending on the number of degrees of freedom in intrinsic subsystem.

According to a general understanding, the non-equilibrium relaxation regime (or called as thermodynamical regime) may also be understood by the Linear Response Theory $(24 ; 26 ; 27)$ provided that the number of degrees of freedom is sufficient large. However, as in the study of quantum dynamical system, the dephasing process only can be understood under the scheme with non-linear coupling interaction, specially for the small number of degrees of freedom. From our results, as shown in Fig. 17, it is clarified that the time scale of dephasing process changes to small with the increasing of the number of degrees of freedom. For the case with two degrees of freedom, the dephasing process lasts for a very long time and dominates the time evolution process of the system. When the number of degrees of freedom increases upto sixteen, the time scale is very small and nonequilibrium relaxation process becomes the main process for energy dissipation. So, in our understanding, for the case with small number of degrees of freedom $\left(N_{d}<8\right)$ where the applicability of Linear Response Theory is still a question of debate $(61 ; 77)$, the dephasing mechanism plays important role for realizing the transport behaviors. When the number of degrees of freedom becomes large (more than sixteen), the thermodynamical mechanism will become a dominant mechanism and there will be no much difference between the Nonlinear and Linear Response Theory.

\section{Entropy evolution of nonequilibrium transport process in finite system}

It is not a trivial discussion how to understand the three regimes as mentioned in above section in a more dynamical way. As mentioned in Sec. II, the transport, dissipative and damping phenomena could be expressed by the collective behavior of the ensemble of trajectories. In the classical theory of dynamical system, the order-to-chaos transition is usually regarded as the microscopic origin of an appearance of the statistical state in the finite system. Since one may express the heat bath by means of the infinite number of integrable systems like the harmonic oscillators whose frequencies have the Debye distribution, it may not be a relevant question whether the chaos plays a decisive role for the dissipation mechanism and for the microscopic generation of the statistical state in a case of the infinite system. In the finite system where the large number limit is not secured, the order-to-chaos transition is expected to play a decisive role in generating some statistical behavior. There should be the relation between the generating the chaotic motion of a single trajectory and the realizing a statistical state for a bundle of trajectories.

\subsection{Nonequilibrium relaxation process \& entropy production 4.1.1 Physical Boltzmann-Gibbs (BG) entropy}

This phenomenon is still represented in the study for clarifying the dynamical relation between the Kolmogorov-Sinai (KS) entropy and the physical entropy for a chaotic conservative dynamical system in classical sense(78), or the status of quantum-classical correspondence for quantum dynamical system(13). The KS entropy is a single number $\kappa$, which is related to the average rate of exponential divergence of nearby trajectories, that is, the summation of all the positive Lyapunov exponents of the chaotic dynamical system 
considered. As for the physical Boltzmann-Gibbs (BG) entropy $S(t)$, the entropy of the second law of thermodynamics, is defined by the distribution function $\rho(t)(68)$ of a bundle of trajectories as:

$$
S(t)=-\int \rho(t) \ln \rho(t) d q d p \prod_{i=1}^{N_{d}} d q_{i} d p_{i},
$$

which depends not only on the particular dynamical system, but also on the choice of an initial probability distribution for the state of that system, which is described by a bundle of trajectories. Therefore the connection between KS entropy and physical (BG) entropy can be considered to given an equivalent relation to that between the chaoticity of a single trajectory and the statistical state for a bundle of trajectories. However, this relation may be not so simple because the KS entropy is the entropy of a single trajectory and in principle, might not coincide with the Gibbs entropy expressed in terms of probability density of a bundle of trajectories.

It has been concluded(78) that the time evolution of $S(t)$ goes through three time regimes: (1) An early regimes where the $S(t)$ is heavily dependent on the details of the dynamical system and of the initial distribution. This regime sometimes is called as the decoherence regime for a Quantum system or dephasing regime for classical system. In this regime, there is no generic relation between $\mathrm{S}(\mathrm{t})$ and $\kappa$; (2) An intermediate time regime of linear increase with slope $\kappa$, i.e., $\left|\frac{d S(t)}{d t}\right| \sim \kappa$, which is called the Kolmogorov-Sinai regime or thermodynamical regime. In this regime, a transition from dynamics to thermodynamics is expected to occur; (3) A saturation regime which characterizes equilibrium, for which the distribution is uniform in the available part of phase space. In accordance with the view of $\operatorname{Krylov}(79)$, a coarse graining process is required here by the division of space.

\subsubsection{Generalized nonextensive entropy \& anomalous diffusion}

It should be mentioned that the physical (BG) entropy $S(t)(89)$ is unable to deal with a variety of interesting physical problems such as the thermodynamics of self gravitating systems, some anomal diffusion phenomena, Lévy flights and distributions, among others(80-83). In order to deal with these difficulties, a generalized, nonextensive entropy form is introduced(84):

$$
S_{\alpha}(t)=\frac{1-\int[\rho(t)]^{\alpha} d q d p \prod_{i=1}^{N_{d}} d q_{i} d p_{i}}{\alpha-1},
$$

where $\alpha$ is called the entropic index, which characterizes the entropy functional $S_{\alpha}(t)$. When $\alpha=1, S_{\alpha}(t)$ reduces to the conventional physical (BG) entropy $S(t)(89)$.

How to understand the departure of $\alpha$ from $\alpha=1$ has been discussed in Refs.(80; 82). From a macroscopic point of view, the diversion of $\alpha$ from $\alpha=1$ measures how that the dynamics of the system do not fulfil the condition of short-range interaction and correlation that according to the traditional wisdom are necessary to establish thermodynamical properties(80). On the other hand, such diversion can be attributed to the mixing (and not only ergodicity) situation in phase space, that is, if the mixing is exponential (strong mixing), the $\alpha=1$ and physical (BG) entropy $\mathrm{S}(\mathrm{t})$ is the adequate hypothesis, whereas the mixing is weak and then nonextensive entropy form should be used(82). We will show in the following that $\alpha \neq 1$ implies the non-uniform distribution in the collective phase space. 

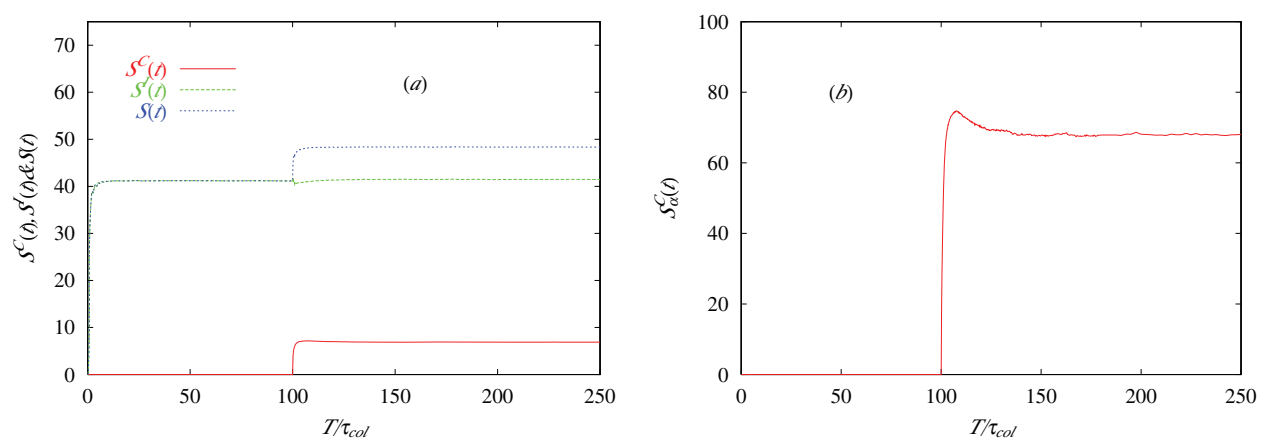

Fig. 18. (a) Physical Boltzmann-Gibbs entropy $S(t)$. Nonextensive entropy $S_{\alpha}(t)$ for collective (b), intrinsic (c) and total phase space (d), for the case with $N_{d}=8$. Entropic index $\alpha=0.7$.

Parameters are the same as Fig. 16.
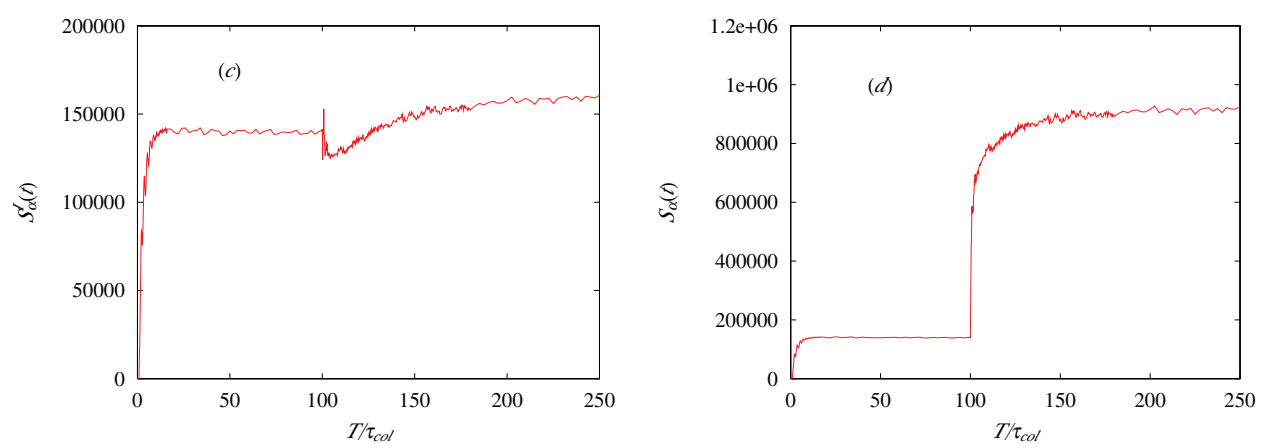

Fig. 18. continued.

It should be very interesting that our simulated energy transfer processes show also three regimes as mentioned in Sec. 3.2.3. Consequently, it is an interesting question whether there is some relation between our numerical simulation and the time evolution of $S(t)$ or $S_{\alpha}(t)$. To understand the underlying connection between these two results, we calculated the entropy evolution process for our system by employing a generalized, nonextensive entropy:

$$
\begin{aligned}
S_{\alpha}^{C}(t) & =\frac{1-\int\left[\rho_{\eta}(t)\right]^{\alpha} d q d p}{\alpha-1}, \\
S_{\alpha}^{I}(t) & =\frac{1-\int\left[\rho_{\xi}(t)\right]^{\alpha} \prod_{i=1}^{N_{d}} d q_{i} d p_{i}}{\alpha-1}
\end{aligned}
$$

where $\rho_{\eta}(t)$ and $\rho_{\xi}(t)$ are the reduced distribution functions (29) of collective and intrinsic subsystems, respectively. For comparison in the following, we also define the physical (BG) 
entropy for collective and intrinsic subsystems as:

$$
\begin{aligned}
& S^{C}(t)=-\int \rho_{\eta}(t) \ln \rho_{\eta}(t) d q d p, \\
& S^{I}(t)=-\int \rho_{\xi}(t) \ln \rho_{\xi}(t) \prod_{i=1}^{N_{d}} d q_{i} d p_{i}
\end{aligned}
$$

Figure 18 shows the comparison between the physical (BG) entropy $S(t)$ in Fig. 18(a) and nonextensive entropy $S_{\alpha}(t)$ Fig. 18(b-d) for collective, intrinsic subsystems and total system for the case with $N_{d}=8$. From this figure, it is understood that there is no entropy produced for collective subsystem before the coupling interaction is activated. However the entropy evaluation process for intrinsic subsystem shows very obviously three regimes both in physical (BG) entropy and in nonextensive entropy. This means that the intrinsic subsystem ( $\beta$-FPU system) can normally diffuse far from equilibrium state to equilibrium state, where the trajectories are uniformly distributed in the phase space. This conclusion is consistent with Ref. (26). After the coupling interaction is switched on, one can see much different situation when one use the physical (BG) entropy or nonextensive entropy in evaluating the entropy production. For intrinsic subsystem, because its time scale is much smaller than collective one, it should be always in time-independent stationary state even after switch-on time $t_{s w}(30)$. This point can be clearly seen from the present simulation in Fig. 18, where there is no change for $S^{I}(t)$ around $t_{s w}$. However, the distribution of trajectories in phase space ought to be changed after $t_{s w}(85)$ which can not be observed by BG entropy. Such the change of the distribution of trajectories in phase space is observed by means of $S_{\alpha}^{I}(t)$ as shown in Fig. 18 (c). We will mention this point furthermore in the following context.

With regard to collective subsystem, our calculated results for $S^{C}(t)$ and for $S_{\alpha}^{C}(t)$ have been shown in Fig. 18(a) and (b). From Fig. 18(a), one may observe that $S^{C}(t)$ increase exponentially to a maximum value just after $t_{s w}$. It is not trivial to answer whether or not this maximum value indicates the stationary state for collective degree of freedom because as mentioned in the last section, the energy interchange between collective and intrinsic subsystems is still continuous in this moment. We can understand this point if we examine the nonextensive entropy $S_{\alpha}^{C}(t)$ in Fig. 18(b). Fig. 18(b) shows that $S_{\alpha}^{C}(t)$ exponentially increases to a maximal value as $S^{C}(t)$, but then almost linearly decrease and finally tends to a saturated time-independent value. The calculated results of second moment of $\left\langle q^{2}\right\rangle$ has shown that such the linearly decreasing process is a superdiffusion process. Those calculated results tells that, for $N_{d}=8$, the time evolution of $S_{\alpha}^{C}(t)$ shows clearly three regimes after $t_{s w}$, says, exponentially increasing regime, linearly decreasing regime and saturated regime.

For understanding the $N_{d}$-dependence of three regimes of transport process, furthermore, we show the comparison of $S_{\alpha}^{C}(t)$ for $N_{d}=2,4,8$ and 16, respectively in Fig. 19 . One may see that the line for the case with $N_{d}=2$ only shows the exponentially increasing behaviour. It has been pointed out(30) that, the dephasing mechanism is mainly contributed to the transport process in the case with $N_{d}=2$. With this point of view, it is easy to understand that the exponentially increasing part corresponds to the dephasing regime. As our understanding, the time scale of dephasing regime mainly depends on the strength of coupling interaction and the chaoticity of intrinsic subsystem, as well as the number of degrees of freedom. In our result, the time scales of dephasing regime for different $N_{d}$ are different with the selection interaction strength $\lambda$ and the largest Lyapunov exponents $\sigma\left(N_{d}\right)$ for intrinsic subsystem. 


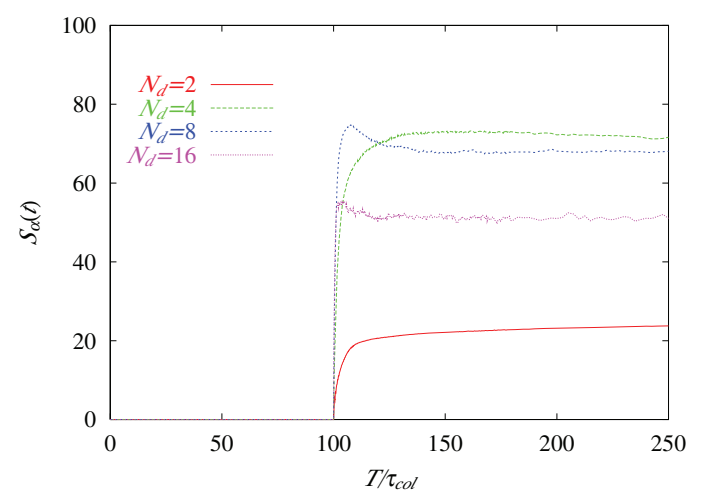

Fig. 19. Comparison of $S_{\alpha}^{C}(t)$ for $N_{d}=2,4,8$ and 16. Parameters are the same as Fig. 18.

With the $N_{d}$ increasing upto 8 , a linearly decreasing process for $S_{\alpha}^{C}(t)$ appears after an exponentially increasing stage. As we understand in last subsection, this should be correspondent to the nonequilibrium relaxation process in which the energy of collective motion irreversibly transfers to the "environment".

It is interesting to mention that there also appears three stages in the entropy production for far-from-equilibrium processes, which is also characterized by using the nonextensive entropy(78). Here it should be noted the point that why the second regime is linearly decreasing, not linearly increasing as V. Latora and M. Baranger's findings(78). The systems considered by V. Latora and M. Baranger(78) and others $(13 ; 80 ; 81)$ are conservative chaotic systems. As we know, for conservative chaotic systems, the entropy will uniquely increase if it is put in a state far from equilibrium state. Our calculated results is consistent with this phenomenon for the total system, which is a conservative system, as shown in Fig. 3 (d), and for intrinsic subsystem, which also can be treated as a conservative system before $t_{s w}$, as shown in Fig. 3 (c). Especially, the collective subsystem is a dissipative system after $t_{s w}$. In the second regime of energy dissipation as described in the last section, the energy of collective motion irreversibly dissipate to intrinsic motion, which should cause the shrink of the distribution of collective trajectories in phase space.

A necessity of using a non-extensive entropy in connecting the microscopic dynamics and the statistical mechanics, and in characterizing the damping phenomenon in the finite system, might suggest us that the damping mechanism in the finite system is an anomalous process, where the usual fluctuation-dissipation theorem is not applicable.

Here it is worthwhile to clarify a relation between an anomalous diffusion and the above mentioned nonextensive entropy expressed by the time evolution of the subsystems with $\alpha<1$, because the non-equilibrium relaxation regime is characterized not by the physical BG entropy but by the nonextensive entropy with $\alpha<1$. Generally, the diffusion process is characterized by the average square displacement or its variance as

$$
\sigma^{2}(t) \sim t^{\mu}
$$

with $\mu=1$ for normal diffusion. All processes with $\mu \neq 1$ are termed anomalous diffusion, namely, subdiffusion for $0<\mu<1$ and superdiffusion for $1<\mu<2$. 


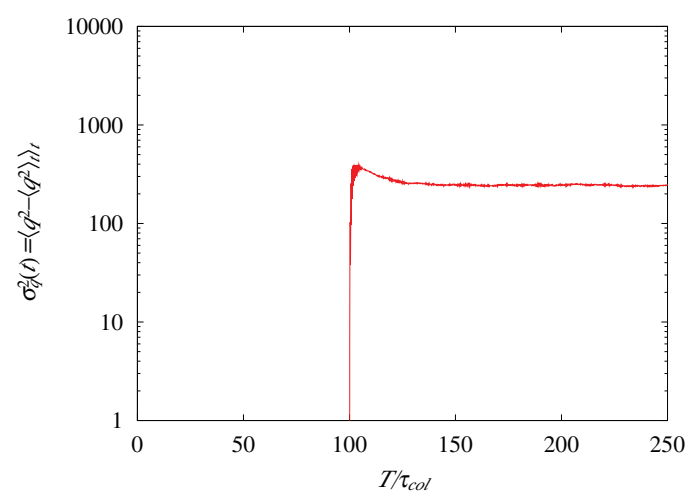

Fig. 20. Time-dependent variance $\sigma_{q}^{2}(t)$ for the case with $N_{d}=8$. Parameters are the same as Fig. 16

We calculate a time-dependent variance of collective coordinate $\sigma_{q}^{2}(t)=\left\langle q^{2}-\left\langle q^{2}\right\rangle_{t}\right\rangle_{t}$ for the case with $N_{d}=8$ as depicted in Fig. 20, which also clearly shows the three stages as discussed above. Here one should mentioned that $\sigma_{q}^{2}(t)$ decreasing from a maximal value to a saturation one in the non-equilibrium relaxation regime, rather than increases from a minimal value to a saturation one as in the conventional approach. In the conventional approach, there does not appear dephasing regime. The collective distribution function $\rho_{\eta}(t)$ spread out from a localized region (say, as $\delta$-distribution) till saturation with an equilibrium Boltzmann distribution. In this case, $\sigma_{q}^{2}(t)$ increases from a minimal value (say, zero) to a saturation one corresponding to the Boltzmann distribution. However, in present case for finite system, $\sigma_{q}^{2}(t)$ exponentially increases from 0 up to a maximal value in dephasing regime as the behavior of entropy $S_{\alpha}^{C}(t)$ in Fig. 18(b) because in this regime, the collective distribution function $\rho_{\eta}(t)$ quickly disperses after the coupling interaction is switched on and tends to cover a ring shape in the phase space. In the second regime of energy dissipation, the collective energy irreversibly dissipates into the intrinsic system with making the distribution of collective trajectories in phase space shrunk until saturation with an equilibrium Boltzmann distribution. It is due to the finite effect that $\sigma_{q}^{2}(t)$ becomes much bigger than its saturation value in the dephasing regime. Therefore, in the second regime, $\sigma_{q}^{2}(t)$ will decreases from this maximal value to a saturation one with shrinking of distribution function of collective trajectories in phase space.

As discussed in Sec. 3.2.3, dephasing regime is the main process for a system with small number of intrinsic degrees of freedom (say, two). A lasting time of this regime decreases with increasing of the number of intrinsic degrees of freedom. When the number of intrinsic degrees of freedom becomes infinite, there might be no dephasing regime. In this case, $\sigma_{q}^{2}(t)$ will show the same behavior as in the conventional approach.

The result of $\sigma_{q}^{2}(t)$ in non-equilibrium relaxation regime can be characterized with the expression

$$
\sigma_{q}^{2}(t)=\sigma_{q}^{2}\left(t_{0}\right)-D\left(t-t_{0}\right)^{\mu_{q}},
$$

where $t_{0}=110 \tau_{c o l}$ is a moment when the dephasing regime has finished, $\sigma_{q}^{2}\left(t_{0}\right)=335.0$ the value of $\sigma_{q}^{2}(t)$ at time $t_{0}$. We fit the diffusion coefficient $\mathrm{D}$ and diffusion exponent $\mu_{q}$ in Eq. 


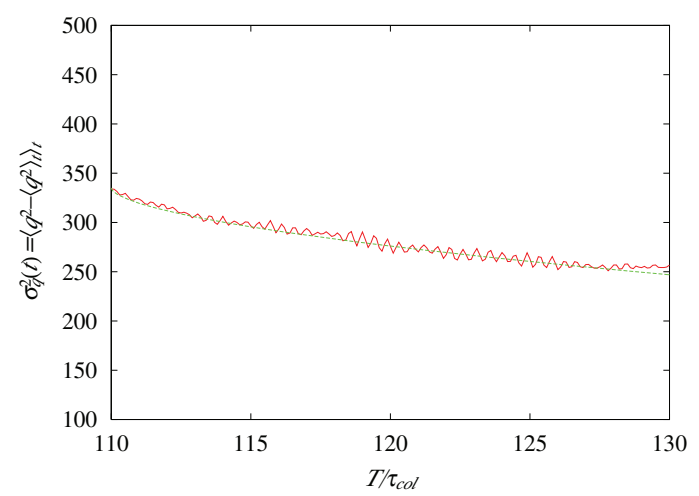

Fig. 21. Time-dependent variance $\sigma_{q}^{2}(t)$ for the case with $N_{d}=8$. Solid line refers to the result of dynamical simulation as shown in Fig. 20; long dashed line refers to the fitting results of Eq. (94) with parameters $D=15.5, \mu_{q}=0.58$.

(94) for the non-equilibrium relaxation regime as plotted in Fig 21. The resultant values are $\mathrm{D}=15.5$ and $\mu_{q}=0.58$, which suggest us that the non-equilibrium relaxation process of a finite system correspond to an anomalous diffusion process.

\subsection{Microscopic dynamics of nonequilibrium process \& Boltzmann distribution}

In order to explore this understanding more deeply, a time development of the collective distribution function $\rho_{\eta}(t)$ in collective $(\mathrm{p}, \mathrm{q})$ space and probability distribution function of collective trajectories which is defined as

$$
\mathrm{P}_{\eta}(\epsilon)=\left.\int \rho_{\eta}(t)\right|_{H_{\eta}(q, p)=\epsilon} d q d p
$$

are shown in Figs. 22 and 23 at different time for $N_{d}=8$. In these figures, it is illustrated how a shape of the distribution function $\rho_{\eta}(t)$ in the collective phase space disperses depending on time. An effect of the damping mechanism ought to be observed when a peak location of the distribution function changes from the outside (higher collective energy) region to the inside (lower collective energy) region of the phase space. On the other hand, a dissipative diffusion mechanism is studied by observing how strongly a distribution function initially (at $t=\tau_{s w}$ ) centered at one point in the collective phase space disperses depending on time.

One may see that from $\mathrm{T}=t_{s w}$ to $110 \tau_{c o l}, \rho_{\eta}(t)$ quickly disperses after the coupling interaction is switched on and tends to cover a ring shape in the phase space. When the distribution function tends to expand over the whole ring shape, the relevant part of each trajectory is not expected to have the same time dependence. Some trajectories have a chance to have an advanced phase, whereas other trajectories have a retarded phase in comparison with the averaged motion under mean-field approximation. This dephasing mechanism is considered to be the microscopic origin of the entropy production in the exponential regime.

The more interesting things appear from $\mathrm{T}=110 \tau_{\text {col }}$ through $\mathrm{T}=140 \tau_{c o l}$. One may see that the distribution function gradually expand to center region from $\mathrm{T}=110 \tau_{c o l}$. The region of maximal probability distribution gradually moves to center, meanwhile the density of 

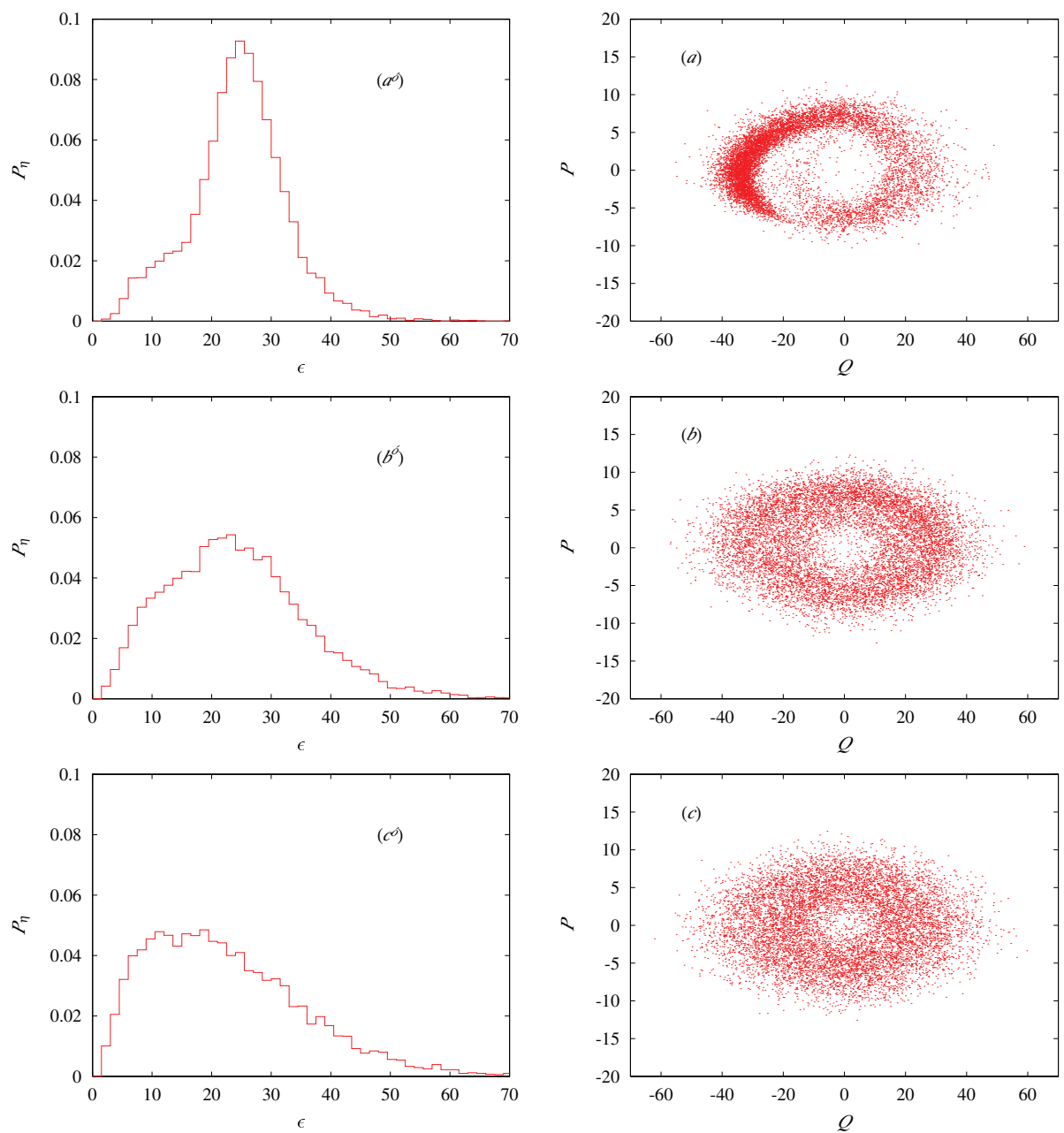

Fig. 22. (a-c) Collective distribution function in (p,q) phase space; $\left(\mathrm{a}^{\dagger}-\mathrm{c}^{\dagger}\right)$ Probability distribution function $\mathrm{P}_{\eta}(\epsilon)$ of collective trajectories at $\mathrm{T}=102.5 \tau_{c o l}, 110 \tau_{c o l}$ and $120 \tau_{c o l}$ for $\mathrm{E}_{\eta}=30$ and $\lambda=0.002$.

distribution in the out ring (as at $\mathrm{T}=110 \tau_{\text {col }}$ ) goes to low. And finally at $\mathrm{T}=160 \tau_{\text {col }}$, one can see the distribution tend to the equilibrium Boltzmann distribution as:

$$
\mathrm{P}_{\eta}(\epsilon) \sim e^{-\beta \epsilon}
$$

After $\mathrm{T}=160 \tau_{c o l}$ to $240 \tau_{\text {col }}$, the distribution function does not practically changed anymore, which is correspondent to the saturated regime. Comparison the distribution in Fig. 23 (f) with the one obtained by the phenomenological transport equation (as Langevin equation) in Fig. 15(b) and in our previous paper(30), one can see that such the distribution is consistent 

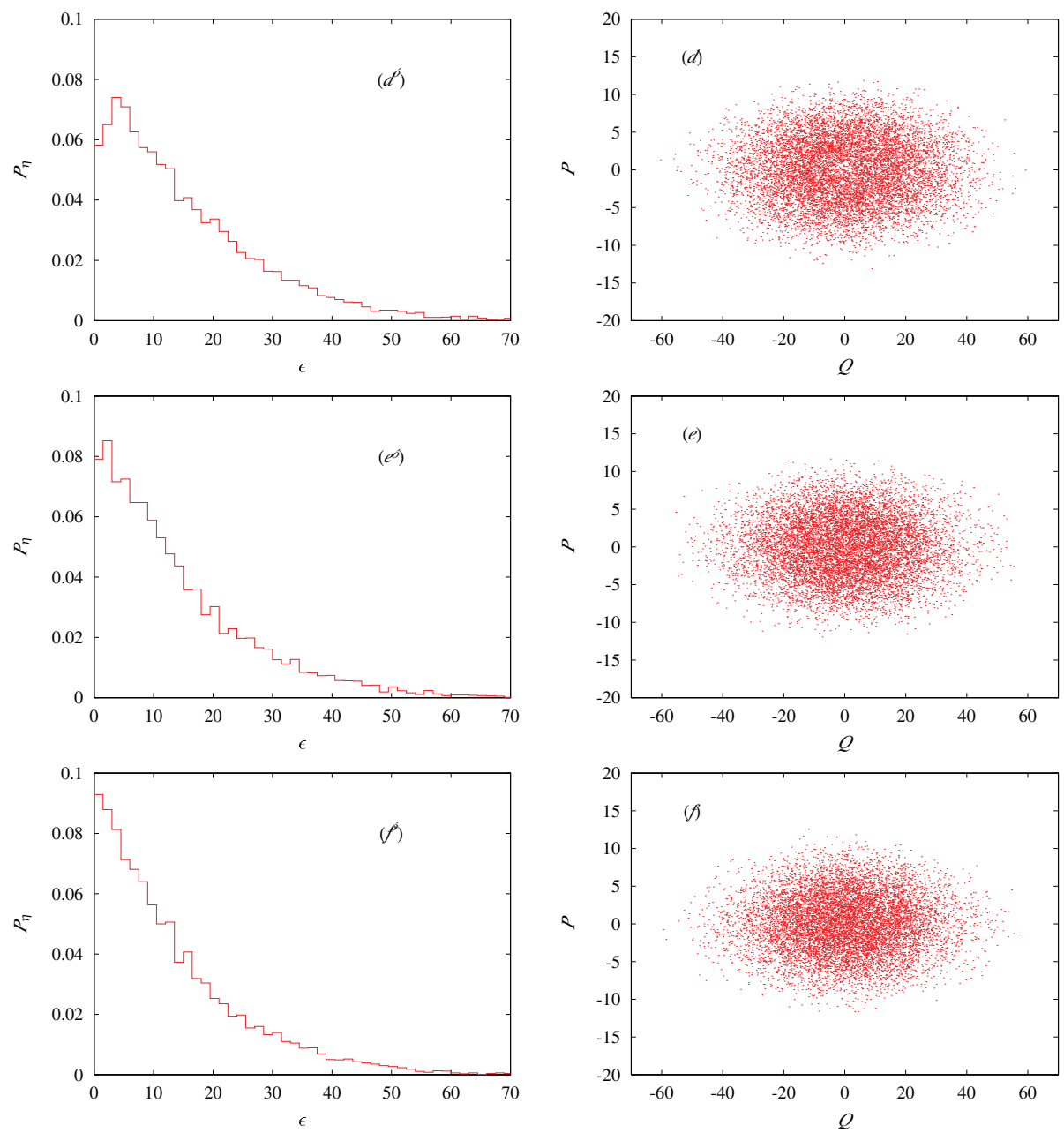

Fig. 23. (d-f) Collective distribution function in $(\mathrm{p}, \mathrm{q})$ phase space; $\left(\mathrm{d}^{\dagger}-\mathrm{f}^{\dagger}\right)$ Probability distribution function $\mathrm{P}_{\eta}(\epsilon)$ of collective trajectories at $\mathrm{T}=140 \tau_{c o l}, 160 \tau_{c o l}$, and $240 \tau_{c o l}$ for $\mathrm{E}_{\eta}=30$ and $\lambda=0.002$.

with the results simulated by Langevin equation. So one can see that a transition from dynamics to thermodynamics occurs indeed and the collective subsystem $\square$ nally reaches to equilibrium state.

At the end of this section, one may conclude that: (i) When the physical BG entropy is used to evaluate the entropy production for the system considered in this work, the three characteristic regimes can not be detected in the collective system. When the non-extensive entropy is used with $\alpha<1.0$, the three dynamical stages, i.e., the dephasing regime, non-equilibrium relaxation regime and equilibrium regime, appear for a relatively large number of intrinsic degrees of freedom as $N_{d} \geq 8$. The second regime may disappear for a small number of degrees of freedom case like $N_{d}=2$. (ii) Since the collective system is a 
dissipative system whose distribution function varies non-uniformly in the non-equilibrium relaxation regime, one has to use the entropy index $\alpha$ different from 1 . (iii) As is shown by the $\sigma_{q}^{2}(t)$ and by $\alpha<1$, the non-equilibrium relaxation process of a finite system considered in this thesis corresponds to the anomalous diffusion process. (iv) The final regime is consistent with the simulation obtained by the phenomenological transport equation. Namely, the statistical state is actually realized dynamically in a finite system which is composed by the collective and intrinsic systems coupled with the nonlinear interaction.

\section{A generalized fluctuation-dissipation relation of collective motion}

\subsection{Derivation of a generalized Fokker-Planck equation}

Now we are at the position to analytically understand why and how the second regime, i.e., thermodynamical regime, appears when the number of degrees of freedom of intrinsic subsystem increases from $N_{d}=2$ to larger one, as $N_{d}=8$. In principle, we can start from the coupled master equation (43), which includes the full information about the time evolution of the two subsystems. However, the coupled master equation (43) is still equivalent to the original Liouville equation (28) and is, in fact, not yet tractable specially for a system far from the stationary states(30).

As was discussed in above sections, when we mainly focus our discussion on the second regime, the intrinsic degrees of freedom can be considered to be in fully developed chaotic situation. In this case, it is reasonable to assume that the effects on the collective system coming from the intrinsic one are mainly expressed by an averaged effect over the intrinsic distribution function (Assumption). Namely, the effects due to the fluctuation part $H_{\Delta}(t)$ are assumed to be much smaller than those coming from $H_{\eta}+H_{\eta}(t)$ and are able to be treated as a stochastic perturbation around the path generated by the mean-field Hamiltonian $H_{\eta}+$ $H_{\eta}(t)^{2}$. In Sec. 3.1.4, a phenomenological transport equation (82) was used in reproducing our simulated results phenomenologically.

$$
M \ddot{q}+\frac{\partial U^{m f}(q)}{\partial q}+\gamma \dot{q}=f(t), \quad q=\frac{\eta+\eta^{*}}{\sqrt{2}},
$$

was used in reproducing our simulated results phenomenologically. Here $U^{m f}(q)$ denotes the potential part of $H_{\eta}+H_{\eta}(t)$, $\gamma$ the friction parameter and $f(t)$ the Gaussian white noise with an appropriate temperature. Since our present main concern is to make clear a relation between the macro-level dynamics organized by the phenomenological equation like Eq. (97) (or like Fokker-Planck equation (118) or the macro-level equation (123) discussed in the following) and the micro-level dynamics by the coupled master equation (43) one step further, we start with the Hamiltonian of collective degree of freedom, which organizes the collective distribution function $\rho_{\eta}(t)$, formally written as:

$$
H_{\eta}^{T}=H_{\eta}+H_{\eta}(t)+\lambda H_{\Delta, \eta}(t)
$$

where $H_{\eta}$ and $H_{\eta}(t)$ are defined in Eq. (64) and Eq. (33), respectively. The main differences between Eq. (97) and Eq. (98) are i) $\gamma$ and $f(t)$ in Eq. (97) are given by hand, ii) $\rho_{\xi}(t)$ specifying the fluctuation effects $H_{\Delta, \eta}(t)$ in Eq.(98) is determined microscopically by Eq. (43). What we are going to discuss in the following, with the aid of Eq. (98), is to understand a change of

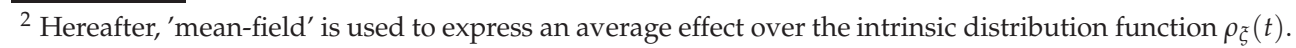


phenomenological parameters in Eqs. (97), (118) or (123) in terms of the fluctuation associated with the microscopic dynamics $\rho_{\xi}(t)$ determined by Eq. (43).

In terms of Eqs. (85) and (86), the coupling interaction can generally expressed as

$$
H_{\text {coupl }}(\eta, \xi)=\lambda \sum_{l} A^{l}(\eta) B^{l}(\xi)
$$

For simplicity, we hereafter discard the summation $l$ in the coupling. The fluctuation Hamiltonian $H_{\Delta, \eta}(t)$ in Eq. (98) then reads

$$
H_{\Delta, \eta}(t)=\phi^{\prime}(t) A(\eta) \quad \phi^{\prime}(t)=B(\xi)-\langle B(\xi)\rangle^{3} .
$$

With the aid of the partial Hamiltonian (98), the distribution function of collective subsystem $\rho_{\eta}(t)$ determined by Eq. (43) then may be explored by using the Liouville equation given by:

$$
\begin{aligned}
\dot{\rho}_{\eta}(t) & =-i \mathcal{L}_{\eta}^{T} \rho_{\eta}(t) \\
& =-i\left(\mathcal{L}_{\eta}+\mathcal{L}_{\eta}(t)+\lambda \mathcal{L}_{\Delta, \eta}(t)\right) \rho_{\eta}(t)
\end{aligned}
$$

where $\mathcal{L}_{\eta}^{T}$ and $\mathcal{L}_{\Delta, \eta}(t)$ are defined as in Eq. (34).

$$
\begin{aligned}
& \mathcal{L}_{\eta}^{T} * \equiv i\left\{H_{\eta}^{T}, *\right\}_{P B}, \\
& \mathcal{L}_{\Delta, \eta}(t) * \equiv i\left\{H_{\Delta, \eta}(t), *\right\}_{P B}
\end{aligned}
$$

Here $\{,\}_{P B}$ denotes a Poisson bracket with respect to the collective variables.

Since, in this section, we are interested in understanding the microscopic dynamics which is responsible for the appearance of the second regime, we can start our discussion from a situation where the dephasing processes have finished, which means that the collective subsystem has reached the situation where the distribution function has the space-reversal symmetry as shown in Figs. 22 and 22 for $t>100 \tau_{c o l}$. Considering that the time scale of dephasing process is much smaller than that of thermodynamical process, the correlation between dephasing and thermodynamical process might be omissible. In this case, the Liouvillian equation (101) can be considered to describe the evolution of distribution function $\rho_{\eta}(t)$ only caused by dissipative mechanism.

Although $H_{\Delta, \eta}(t)$ contains the intrinsic variables, in the present formulation, the fluctuation $H_{\Delta, \eta}(t)$ should be considered to be a time dependent stochastic force expressed as $\phi^{\prime}(t)$ in Eq. (100), and a stochastic average is obtained by taking the integration over the intrinsic variables with a weight function $\rho_{\xi}(t)$. Here it should be noticed that the Liouville equation (101) is an approximation to Eq. (43). Since our present aim is to explore how the effects on the collective system coming from the intrinsic fluctuation $\phi^{\prime}(t)$ change depending on the number of intrinsic degrees of freedom as simple as possible, we start with Eq. (101) rather than Eq. (43). Namely, the collective fluctuation effects originated from $A(\eta)-\operatorname{Tr}_{\eta} A(\eta) \rho_{\eta}$ on the intrinsic system ought to be disregarded, because we are now studying the average dynamics of collective motion.

\footnotetext{
${ }^{3}$ Except specific definition, thereafter, the average is obtained by taking the integration over the intrinsic variables with a weight function $\rho_{\xi}(t)$ at time $t$, say $<*>=\operatorname{Tr}_{\xi} * \rho_{\xi}(t)$.
} 
It should be emphasized that we formally express the partial Hamiltonian and Liouville equation of collective degree of freedom as Eqs. (98) and (101). However, in order to closely relate our analysis carried out in this section with our numerical simulation as shown in last two section, the Liouville equation (101) will not be used to determine $\rho_{\eta}(t)$, but be used only to understand what happens in the collective distribution function $\rho_{\eta}(t)$, which is numerically obtained by integrating the canonical equations of motion (69) with the Hamiltonian in Eq. (25).

Let us start our discussion just after the dephasing process has finished. Eq. (101) may now be a linear stochastic equation with fluctuation term $\mathcal{L}_{\Delta, \eta}(t)$. When the fluctuation part is regarded as a perturbation, one may introduce the mean-eld propagator

$$
G_{\eta}\left(t, t^{\prime}\right)=\operatorname{Texp}\left\{-i \int_{t^{\prime}}^{t}\left[\mathcal{L}_{\eta}+\mathcal{L}_{\eta}(\tau)\right] d \tau\right\}
$$

which describes an average time-evolution of the collective system.

Under the help of the mean-eld propagator $G_{\eta}\left(t, t^{\prime}\right)$ and taking the stochastic average over $\rho_{\xi}(t)$, one may obtain the master equation for $\rho_{\eta}(t)$ from Eq. (101), as

$$
\begin{aligned}
\rho_{\eta}(t)= & -i\left\{\mathcal{L}_{\eta}+\mathcal{L}_{\eta}(t)\right\} \rho_{\eta}(t) \\
& -\lambda^{2} \int_{0}^{\infty} d \tau\left\langle\left\langle\mathcal{L}_{\Delta, \eta}(t) G_{\eta}(t, t-\tau) \mathcal{L}_{\Delta, \eta}(t-\tau)\right\rangle\right\rangle G_{\eta}(t-\tau, t) \rho_{\eta}(t)
\end{aligned}
$$

where a symbol $\ll \cdots \gg$ denotes a cumulant defined as:

$$
\ll \mathcal{A B} \gg \equiv<\mathcal{A B}>-<\mathcal{A}><\mathcal{B}>
$$

which relates to the average $<*>_{t}=\operatorname{Tr}_{\xi} * \rho_{\xi}(t)$. A derivation of Eq. (104) is given in Appendix 10.

In getting Eq. (104) from Eq. (142), we have supposed ${ }^{4}$ that the collective distribution function $\rho_{\eta}(t)$ evolves through the mean-field Hamiltonian $H_{\eta}+H_{\eta}(t)$ from $t-\tau$ to $t$. This is because the fluctuation effects are so small as to be treated as a perturbation around the path generated by the mean-field Hamiltonian $H_{\eta}+H_{\eta}(t)$, and are sufficient to be retained in Eq. (104) up to the second order in $\lambda$. Under the assumption of a weak coupling interaction and of a finite correlation time $\tau_{c}$

$$
\left\langle\left\langle\phi^{\prime}(t) \phi^{\prime}\left(t^{\prime}\right)\right\rangle\right\rangle=0 \quad \text { for } \quad\left|t-t^{\prime}\right|>\tau_{c}
$$

an upper limit in the time integration in Eq. (104) may be extended to $\infty$.

Eq. (104) is valid upto the second-order cumulant expansion. Here

$$
\left\langle\left\langle\phi^{\prime}(t) \phi^{\prime}\left(t^{\prime}\right)\right\rangle\right\rangle=\left\langle\phi^{\prime}(t) \phi^{\prime}\left(t^{\prime}\right)\right\rangle-\left\langle\phi^{\prime}(t)\right\rangle\left\langle\phi^{\prime}\left(t^{\prime}\right)\right\rangle
$$

The mean- eld propagator operator $G_{\eta}(t, t-\tau)$ provides the solution of unperturbed equation. That is, there holds a relation

$$
f(\eta, t)=G_{\eta}(t, t-\tau) f(\eta, t-\tau),
$$

\footnotetext{
$\overline{4}$ as Condition I in Sect. 2.3.
} 
provided $f(\eta, t)$ satisfies a relation

$$
\frac{\partial f(\eta, t)}{\partial t}=-i\left(\mathcal{L}_{\eta}+\mathcal{L}_{\eta}(t)\right) f(\eta, t)
$$

Since the Liouville equation (107) is equivalent to the canonical equation of motion given by

$$
\begin{aligned}
i \dot{\eta}_{a} & =\frac{\partial\left(H_{\eta}+H_{\eta}(t)\right)}{\partial \eta_{a}^{*}}, \\
i \dot{\eta}_{a}^{*} & =-\frac{\partial\left(H_{\eta}+H_{\eta}(t)\right)}{\partial \eta_{a}}
\end{aligned}
$$

there holds a relation

$$
f(\eta, t)=f\left(\eta^{t-\tau}, t-\tau\right)\left|\frac{d \eta^{t-\tau}}{d \eta}\right|=G_{\eta}(t, t-\tau) f(\eta, t-\tau)
$$

$\left|d \eta^{t-\tau} / d \eta\right|$ being a Jacobian determinant.

Using the above relation, Eq. (104) can be simplified as

$$
\begin{aligned}
\dot{\rho}_{\eta}(t)= & -i\left\{\mathcal{L}_{\eta}+\mathcal{L}_{\eta}(t)\right\} \rho_{\eta}(t) \\
& -\lambda^{2} \int_{0}^{\infty} d \tau\left|\frac{d \eta^{t-\tau}}{d \eta}\right|\left\langle\left\langle\mathcal{L}_{\Delta, \eta}(t) \mathcal{L}_{\Delta, \eta}^{-\tau}(t-\tau)\right\rangle\right\rangle\left|\frac{d \eta}{d \eta^{t-\tau}}\right| \rho_{\eta}(t)
\end{aligned}
$$

where

$$
\begin{aligned}
& \mathcal{L}_{\Delta, \eta}(t) * \equiv i \phi^{\prime}(t)\{A(\eta), *\}, \\
& \mathcal{L}_{\Delta, \eta}^{-\tau}(t-\tau) * \equiv i \phi^{\prime}(t-\tau)\left\{A\left(\eta^{t-\tau}\right), *\right\} .
\end{aligned}
$$

With Hamiltonians $H_{\eta}$ and $H_{\eta}(t)$ as defined in Eq. (64) and (33), one may easily get an analytical form of mapping $\eta \rightarrow \eta^{\tau}$ by solving the unperturbed equation (108)

$$
\begin{aligned}
& q(\tau)=q \cos \omega^{\prime} \tau+\frac{p}{M \omega^{\prime}} \sin \omega^{\prime} \tau \\
& p(\tau)=-q M \omega^{\prime} \sin \omega^{\prime} \tau+p \cos \omega^{\prime} \tau
\end{aligned}
$$

where we have used the relation (85) and the relation defined by

$$
\omega^{\prime 2}=\omega^{2}+\frac{2 \lambda\left\langle\left\{q_{1}^{2}-q_{1,0}^{2}\right\}\right\rangle}{M}
$$

The Jacobian determinant of this mapping reads

$$
\left|\frac{d \eta^{-\tau}}{d \eta}\right|=\left|\frac{d \eta}{d \eta^{-\tau}}\right|=1
$$


because $\omega^{\prime}$ does not practically depend on time. In terms of the coupling interaction (86), the fluctuation Hamiltonian $H_{\Delta, \eta}(t)$ in Eq. (100) can be explicitly written as

$$
\begin{aligned}
& H_{\Delta}^{\eta}(t)=\phi^{\prime}(t)\left\{q^{2}-q_{0}^{2}\right\} \\
& \phi^{\prime}(t)=\left\{q_{1}^{2}-q_{1,0}^{2}\right\}-\left\langle\left\{q_{1}^{2}-q_{1,0}^{2}\right\}\right\rangle .
\end{aligned}
$$

With the help of Eq. (115), the culmulant in Eq. (110) can therefore be expressed

$$
\left\langle\left\langle\mathcal{L}_{\Delta, \eta}(t) \mathcal{L}_{\Delta, \eta}^{-\tau}(t-\tau)\right\rangle\right\rangle=-\langle\langle\phi(t) \phi(t-\tau)\rangle\rangle q \frac{\partial}{\partial p} q(t-\tau) \frac{\partial}{\partial p(t-\tau)}
$$

where $\phi(t)=2 \phi^{\prime}(t)$. Considering $\left(\eta^{-\tau}\right)^{\tau}=\eta$ and using Eqs. (113), one gets

$$
\begin{aligned}
q(t-\tau) \frac{\partial}{\partial p(t-\tau)} & =\left(q \frac{\sin \omega^{\prime} \tau \cos \omega^{\prime} \tau}{M \omega^{\prime}}-p \frac{\sin ^{2} \omega^{\prime} \tau}{M^{2} \omega^{\prime 2}}\right) \frac{\partial}{\partial q} \\
& +\left(q \cos ^{2} \omega^{\prime} \tau-p \frac{\sin \omega^{\prime} \tau \cos \omega^{\prime} \tau}{M \omega^{\prime}}\right) \frac{\partial}{\partial p}
\end{aligned}
$$

Finally, Eq. (110) can be explicitly written as:

$$
\frac{\partial \rho_{\eta}(t)}{\partial t}=\left\{\mathcal{L}_{e f f}+\lambda^{2}\left(\beta_{1} q \frac{\partial}{\partial p}\left(q \frac{\partial}{\partial q}-p \frac{\partial}{\partial p}\right)+\beta_{2} q^{2} \frac{\partial}{\partial p^{2}}-\beta_{3} q \frac{\partial}{\partial p} p \frac{\partial}{\partial q}\right)\right\} \rho_{\eta}(t)
$$

where

$$
\mathcal{L}_{e f f}=-\frac{p}{M} \frac{\partial}{\partial q}+M \omega^{\prime 2} q \frac{\partial}{\partial p}
$$

is an effective unperturbed (mean-field) Liouvillian of the collective system. The parameters $\beta_{1}, \beta_{2}$ and $\beta_{3}$ are expressed as the Fourier transformations of the correlation functions of intrinsic system:

$$
\begin{aligned}
& \beta_{1}=\frac{1}{M \omega^{\prime}} \int_{0}^{\infty} d \tau\langle\langle\phi(t) \phi(t-\tau)\rangle\rangle \cos \omega^{\prime} \tau \sin \omega^{\prime} \tau \\
& \beta_{2}=\int_{0}^{\infty} d \tau\langle\langle\phi(t) \phi(t-\tau)\rangle\rangle \cos ^{2} \omega^{\prime} \tau \\
& \beta_{3}=\frac{1}{M^{2} \omega^{\prime 2}} \int_{0}^{\infty} d \tau\langle\langle\phi(t) \phi(t-\tau)\rangle\rangle \sin ^{2} \omega^{\prime} \tau
\end{aligned}
$$

Eq. (118) is the two-dimensional Fokker-Planck equation. The first term on the right-hand side of Eq. (118) represents the contribution from the mean-field part $H_{\eta}+H_{\eta}(t)$, and the last three terms represent contributions from the dynamical fluctuation effects $H_{\Delta, \eta}$. The parameters $\beta_{1}, \beta_{2}$ and $\beta_{3}$ establish the connection between the macro-level dynamical evolution of collective system and the micro-level fluctuation of intrinsic one. 


\subsection{Fluctuation-dissipation relation \& correlation functions}

With the same definition of ensemble average $\mathrm{e}^{5}$ as Eq. (75), one can find the identity

$$
\frac{d\langle X\rangle}{d t}=\int X \frac{d \rho_{\eta}(t)}{d t} d q d p
$$

which is valid for any collective variable which does not explicitly depend on time. When one inserts the equation (118) into (120), and evaluates the individual term by observing the standard rule:

$$
\int A\{B, C\}_{P B} d q d p=\int\{A, B\}_{P B} C d q d p
$$

one can obtain the equation of first moment:

$$
\begin{aligned}
& \frac{d\langle q\rangle}{d t}=\frac{\langle p\rangle}{M} \\
& \frac{d\langle p\rangle}{d t}=-\frac{\lambda \beta_{3}}{M}\langle p\rangle-\left[M \omega^{\prime 2}-\lambda^{2} \frac{\beta_{1}}{M}\right]\langle q\rangle
\end{aligned}
$$

or in a compact way as

$$
\begin{aligned}
& M\langle\ddot{q}\rangle+\Gamma\langle\dot{q}\rangle+\left[M \omega^{\prime 2}-\lambda^{2} \frac{\beta_{1}}{M}\right]\langle q\rangle=0 \\
& \Gamma=\frac{\lambda \beta_{3}}{M}=\frac{\lambda}{M^{3} \omega^{\prime 2}} \int_{0}^{\infty} d \tau\langle\langle\phi(t) \phi(t-\tau)\rangle\rangle \sin ^{2} \omega^{\prime} \tau
\end{aligned}
$$

Here the parameter $\Gamma$ (or $\beta_{3}$ ) represents the damping effects on collective dynamical motion coming from the fluctuation interaction, which originates from the chaoticity of intrinsic subsystem. Eq. (124) can be regarded as a phenomenological Fluctuation-Dissipation relation and the damping phenomenon (described by $\beta_{3}$ or $\Gamma$ ) implies that the energy is irreversibly dissipated from collective subsystem and absorbed by intrinsic one.

Applying the similar procedures for obtaining Eq. (123), one may derive an equation of motion for the second moments

$$
\frac{d}{d t}\left[\begin{array}{c}
\langle q q\rangle \\
\langle p p\rangle \\
\langle q p\rangle
\end{array}\right]=\left[\begin{array}{ccc}
0 & 0 & \frac{2}{M} \\
4 \lambda^{2} M^{2} \omega^{\prime 2} \beta_{3}+2 \lambda^{2} \beta_{2} & -4 \lambda^{2} \beta_{3} & -2 M \omega^{\prime 2}-2 \lambda \beta_{0} \\
-M^{2} \omega^{\prime 2}-\lambda \beta_{0}+2 \lambda^{2} \beta_{1} & \frac{1}{M} & -4 \lambda^{2} \beta_{3}
\end{array}\right]\left[\begin{array}{c}
\langle q q\rangle \\
\langle p p\rangle \\
\langle q p\rangle
\end{array}\right]
$$

A real eigenvalue of the matrix in above equation indicates an instability of collective trajectory which is caused by the chaoticity of intrinsic trajectory through fluctuation Hamiltonian $H_{\Delta, \eta}(48)$.

Equations (118), (123) and (125) have set up an phenomenological relation among the micro-level properties of the intrinsic subsystem and the macro-level time evolution of collective subsystem through microscopic correlation functions.

Employing the numerical simulation results for Eq. (69), we calculate the correlation function $\langle\langle\phi(t) \phi(t-\tau)\rangle\rangle$ at $t=120 \tau_{c o l}$ for the case with $N_{d}=2,4$ and 8 as shown in Fig. 24. Generally

\footnotetext{
${ }^{5}$ Ensemble average means integration over the collective variables with a weight function $\rho_{\eta}(t)$ at time $\mathrm{t}$, say $<*>=\operatorname{Tr}_{\eta} * \rho_{\eta}(t)$.
} 


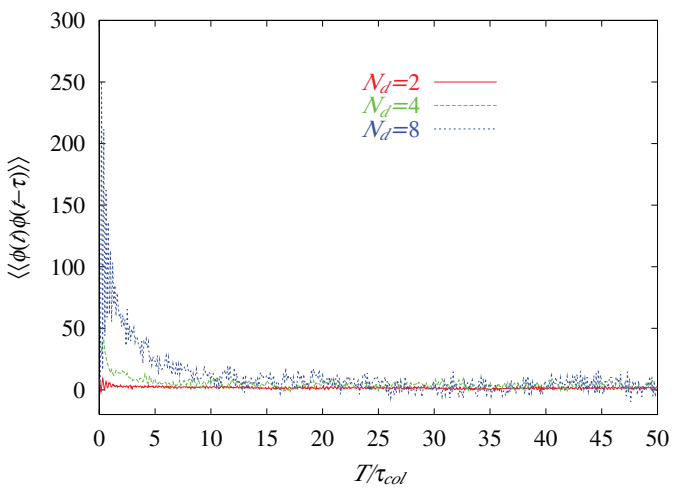

Fig. 24. Correlation function at $t=120 \tau_{c o l}$ for the case with $N_{d}=2,4$ and 8 . Parameters are the same as Fig. 16.

speaking, the correlation function $\langle\langle\phi(t) \phi(t-\tau)\rangle\rangle$, as well as the parameters $\beta_{1}, \beta_{2}$ and $\beta_{3}$ may have a strong time dependence, when the intrinsic system undergoes a drastic change like in the dephasing regime. Since, in the present context, we want to understand why the second regime, i.e., thermodynamical regime, appears when the number of degrees of freedom of intrinsic subsystem increases from $N_{d}=2$ to larger one, as $N_{d}=8$, it is reasonable for us to select a moment, just after the dephasing process has finished. From Figs. $16 \sim 23$, it can be seen that $t=120 \tau_{c o l}$ is just corresponds to such the moment. This is the reason why we calculate the correlation function at $t=120 \tau_{c o l}$.

From Fig. 24, one can see that the correlation function for the case with $N_{d}=2$ is very weak and oscillates around $\langle\langle\phi(t) \phi(t-\tau)\rangle\rangle_{t}=0$. In this case, the main influence of the intrinsic subsystem on collective one acts as a source of dephasing, and has finished before $t=120 \tau_{\text {col }}$ . As $N_{d}$ increases, the magnitude of the correlation function becomes large and behaves like a "colored noise" with finite correlation time $\tau_{c}$ :

$$
\langle\langle\phi(t) \phi(t-\tau)\rangle\rangle \sim e^{-\frac{\tau}{\tau_{c}}}
$$

From this calculation, the correlation function seems to reach a $\delta$ function which represents a "white noise" when $N_{d}$ increases to infinite. This results verify our understanding as shown in Sec. 3.2 and 4:

- The dephasing regime is the main mechanism for the small number of freedom (say, two) case.

- Both the dynamical description and conventional transport approach can provide us with almost same macro- and micro-level mechanisms only for the system with very large number of degrees of freedom, however, for the finite system, the statistical relaxation is an anomalous diffusion and the fluctuation effects have $\square$ nite correlation time.

In fact, the approximation of "white noise" is never exactly realized for the realistic physical system, specially for the finite system. What must be done is to consider the noise and the physical system within which, or upon which, it is operating together. Specifically, the finite-time correlation of noise must be taken into account. 


\begin{tabular}{|r|rrrr|}
\hline$N_{d}$ & 2 & 4 & 8 & 16 \\
\hline$\beta_{3}$ & 0.201 & 0.530 & 0.820 & 1.773 \\
\hline
\end{tabular}

Table 2. Calculated values of parameter $\beta_{3}$ at $t=120 \tau_{\text {col }}$ for the case with $N_{d}=2,4,8$ and 16, respectively.

Using above results of correlation function, we calculate the parameter $\beta_{3}$ as shown in Table 2. As is obviously seen, the damping effects on collective dynamical motion will increases when the number of degree of freedom increases. For the case with small number of degree of freedom, the damping effects are too small to make the second regime realized. This understanding is consistent with our conclusion stated in Sec. 3.1.4-3.1.5 and in our previous paper(30), where we pointed out that the dephasing mechanism is essential for the damping of the collective energy in the case with two degrees of freedom. However for the case with larger number of degree of freedom as $N_{d}=8$ or more, the damping effects become appreciable and make the collective system thermodynamically relaxed to an equilibrium state. This result provides us with a microscopically understanding on how and why the second and third regimes are realized for the collective system coupled to the intrinsic system with an appropriately large number of degrees of freedom.

At the end of this section, we want to discuss on the fluctuation-dissipation relation of collective motion (124). As mentioned in Sec. 3.2.2, the energy equipartition among every degrees of freedom can be expected in the final regime for the case with relatively larger number of degrees of freedom, as $N_{d}=8$. This situation just corresponds to a case where the conventional transport equation is applied and the fluctuation-dissipation relation of the collective motion is expected.

Since the collective energy is given by

$$
\langle E\rangle=\frac{\left\langle p^{2}\right\rangle}{2 M}+\frac{1}{2} m \omega^{3}\left\langle q^{2}\right\rangle,
$$

which is derived from Eq. (64), one may evaluate a rate of collective energy change as

$$
\frac{d\langle E\rangle}{d t}=\left[\frac{4 \lambda^{2} M^{2} \omega^{\prime 2} \beta_{3}+2 \lambda^{2} \beta_{2}}{2 M}\langle q q\rangle-\frac{4 \lambda^{2} \beta_{3}}{2 M}\langle p p\rangle\right]
$$

by using Eq. (125). Since the energy interchange between the two subsystems is supposed to have finished on the average in the third regime, that is, from Eq. (127), the relation

$$
\left[\left(2 M^{2} \omega^{\prime 2} \beta_{3}+\beta_{2}\right)\langle q q\rangle-2 \beta_{3}\langle p p\rangle\right]=0
$$

should be satisfied. Figure 25 shows the results of the right-hand-side of equation (127). It is clearly seen that a relation (128) is actually satisfied on the third regime.

To summarize this section, one may get the following conclusions. The damping mechanism caused by fluctuation interaction is the main reason of the appearance of the thermodynamical process. When the number of intrinsic degrees of freedom is relatively large (as $N_{d}=8$ ), the damping mechanism makes the realization of the thermodynamical process and the saturated 


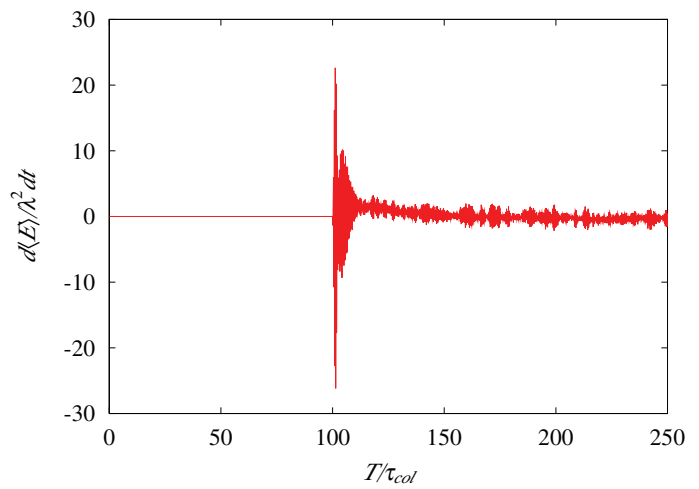

Fig. 25. Rate of collective energy change in Eq. (127). Correlation function $\langle\langle\phi(t) \phi(t-\tau)\rangle\rangle$ is calculated at $t=240 \tau_{c o l}$ for $N_{d}=8$. Parameters are the same as Fig. 16

situation. In this case, the traditional Fokker-Planck equation is safely used in describing the thermodynamical process, and a Fluctuation-Dissipation relation is well realized.

It should be pointed out that, in this section, we have used the microscopic master equation (104) rather than the coupled master equation (43), in order to analytically study the microscopic dynamics responsible for the macro-level dissipative motion as plainly as possible. Although the similarity between Eq. (104) and Eq. (43) is obvious, the former does not includes the effects coming from the response functions which are also known to play an important role in understanding an interrelation between the micro-level and macro-level dynamics. Since the effects of the response function was explored in our previous paper(21), and since the former is convenient to show the physical role of the correlation function clearly, in this section, we have started our analytical discussion from Eq. (104). Apparently, it is our next subject to study the role of the dynamical correlation and dynamical response functions more deeply and more comprehensive way.

\section{Linear and nonlinear coupling}

According to the SCC method, which has been developed to optimally divide the total space into the relevant and irrelevant subspaces, there should not remain any linear coupling interaction between two spaces. In other words, one may optimally divide the total system into the two decoupled subsystems by using such a dynamical condition that the linear coupling between them should be eliminated. Since a ratio between the time scale of the well developed collective motion and that of the single-particle motion is typically less than one order of magnitude in such a finite system as nucleus, it is a very important task to carefully study how the relevant degrees of freedom are distinguished from the rest degrees of freedom. On the basis of the SCC method, one may state that the separation of the total system into two subsystems coupled with a linear interaction has no physical meaning in a finite system, because a choice of the coordinate system , i.e., a separation between the relevant and irrelevant coordinates remains arbitrary when there remains a linear coupling between them. This statement is easily recognized when one remembers that the harmonic oscillators coupled with the linear interaction reduce to the uncoupled harmonic oscillators by a proper choice of the coordinate system. Here, we do not intend to extend the above statement for 

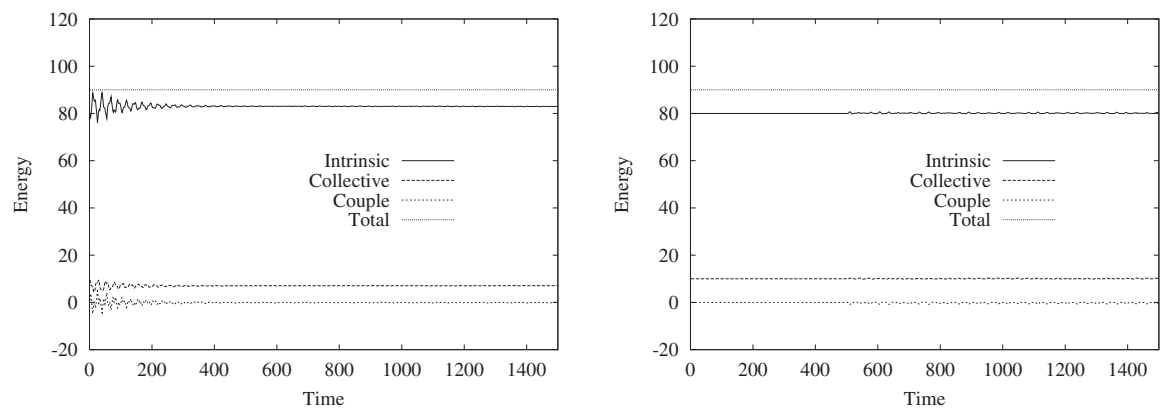

Fig. 26. The distribution of the partial Hamiltonian $\left\langle H_{\eta}\right\rangle,\left\langle H_{\xi}\right\rangle,\left\langle H_{\text {coupl }}\right\rangle$ and $\langle H\rangle$ for $N_{d}=8$ and $\Delta=0.02$

the infinite system, because there is many order of magnitude difference between a time scale of the macroscopic motion and that of the microscopic one, and there are huge number of degrees of freedom in the infinite irrelevant system.

In order to explore the different effects between the linear and nonlinear coupling interactions on the dissipative process, we have made a numerical simulation for the $\beta$ Fermi-Pasta-Ulam ( $\beta$-FPU) model described in Sec. 3.2.1. The collective $H_{\eta}$, intrinsic $H_{\xi}$ are the same as in Sec. 3.2.1, but a coupling $H_{\text {coupl }}$ Hamiltonians is given as

$$
H_{\text {coupl }}=\Delta q \cdot q_{1} \text {. }
$$

The numerical results are illustrated in Fig. 26. In Fig. 26(a), the coupling is switch on from the beginning, whereas in Fig. 26(b) it is switch on at $\tau_{s w}=500 \tau_{\text {in }}$ when a chaotic situation has been well realized in the intrinsic system. In Fig. 26(a), one may observe small energy transfer from the collective to the intrinsic system when the system reach its stationary state at $t \approx 400 \tau_{i n}$. Namely $\left\langle H_{\xi}>\right.$ becomes a little bit greater than 80 and $<H_{\eta}>$ less than 10 . Before reaching their stationary states, especially at the early stage at $t \leq 100 \tau_{\text {in }}$, there are violent energy exchange between the collective and intrinsic systems. Since it is not allowed to apply some statistical treatment for the intrinsic system in this early stage when no stationary state is realized for it, there might be no reason to apply the Langevin type equation for a case in Fig. 26(a). In other words, the above energy transfer may not be understood in terms of the macroscopic terms. When one switches on the coupling after the chaotic state has been realized in the intrinsic system, there is almost no energy dissipation in the collective motion as is seen from Fig. 26(b).

An essential difference between the linear and nonlinear coupling cases may be understood as follows: As is seen from Eq. (77), the coupling $H_{\text {coupl }}$ produces the mean field potential $H_{\eta}(t)$ in the case of the nonlinear coupling, because the second moment $<\sum_{i=1}^{2}\left\{q_{i}^{2}+p_{i}^{2}\right\}>$ has some value when the intrinsic system reaches some stationary state. It is recognized from Eq. (81) that this average effect plays a decisive role to define an amount of transferred energy from the collective system to the environment, like the friction force. On the other hand, $H_{\text {coupl }}$ does not produce any averaged effects on the collective motion in the case with the linear coupling, because there holds a relation $\left\langle q_{i}\right\rangle=0$ when the statistical state is realized in the intrinsic system. With regards to the $\beta$-FPU model, one may conclude that the 

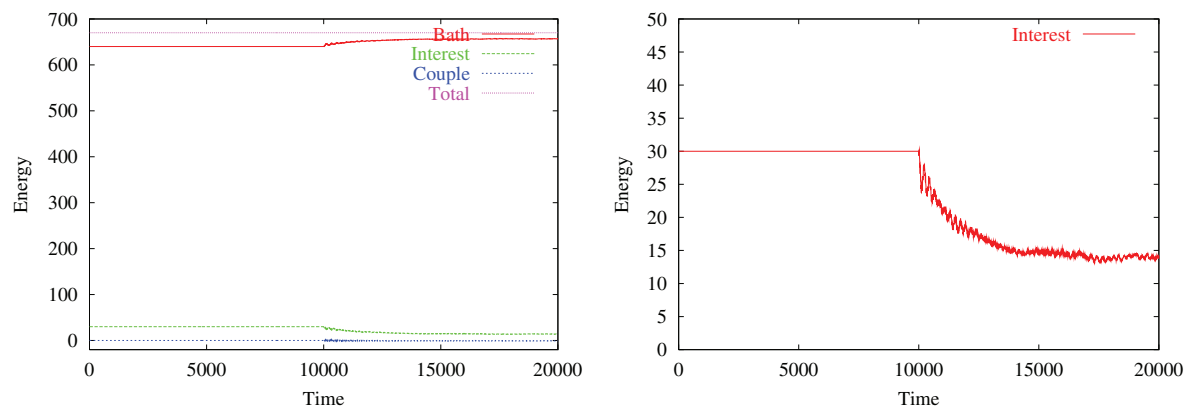

Fig. 27. The distribution of the partial Hamiltonian $\left\langle H_{\eta}\right\rangle,\left\langle H_{\xi}\right\rangle,\left\langle H_{\text {coupl }}\right\rangle$ and $\langle H\rangle$ for $\Delta=0.02$, $N_{d}$ is 64 which almost can be treated as the case with infinite number of degrees of freedom.

energy dissipation phenomena may not be expected for a finite system, although the other main numerical results described in Ref. (26) have been reproduced.

However, as mentioned in Sec. 3.2.3, the nonequilibrium relaxation regimen (or called as thermodynamical regimen) may also be understood by the Linear Response Theory (24; 26; 27) provided that the number of degrees of freedom is sufficient large. Our numerical results, as shown in Fig. 27, confirm this conclusion. The number of degrees of freedom $N_{d}$ is chosen as 64, which almost can be considered as infinite. There still remain some unsolved questions for the case with a linear coupling interaction, such as: what is the microscopic reason for energy dissipation in the case with very large number of degrees of freedom (as $N_{d}>64$ ); what is the difference in microscopic dynamics between the cases with very large and small number of degrees of freedom, and furthermore, why a linear coupling interaction can remain between relevant and irrelevant degrees of freedom for producing the Ohmic dissipation for relevant motion, why the linear response theory can be applied for the micro-variables which have a very short time scale in comparison with that for the macro-variables. Such the problem will be investigated in our further works.

\section{Summary}

We have systematically discussed the characteristic features of non-equilibrium processes for a microscopic Hamilton system with finite degrees of freedom without introducing any statistical ansatz.

The total system is self-consistently and optimally divided into the relevant and irrelevant degrees of freedom in a way consistent with the underlying microscopic dynamics for aiming to properly characterize the collective motion, where the linear coupling between the relevant and irrelevant degrees of freedom is eliminated by the maximally-decoupled coordinate system. This division in the degrees of freedom turns out to be very important for exploring the energy dissipation process and nonlinear dynamics between the collective and intrinsic modes of motion.

A macroscopic transport equation has been derived from the fully microscopic master equation for a microscopic system composed of the one collective degree of freedom system coupled to an intrinsic system with finite degrees of freedom through a weak interaction, by clarifying necessary underlying conditions. 
It has been found that the macro-level statistical aspects are strongly related to an appearance of micro-level chaotic motion, and the dissipation of collective motion is realized through dephasing, statistical relaxation and equilibrium regimes. Dominant stage changes depending on the number of intrinsic degrees of freedom. It has been clearly clarified that both the dynamical description and conventional transport approach can provide us with almost same macro- and micro-level mechanisms only for the system with very large number of degrees of freedom, however for the finite system, the statistical relaxation is anomalous diffusion and the fluctuation effects have finite correlation time.

It is interesting to notice that the nonequilibrium relaxation regime is characterized not by the usual BG entropy, but by the nonextensive entropy with $\alpha<1$, which has been used to extend the thermodynamics to a system with a fractal structure.

Under the help of analytical analysis, we have shown that the energy transport process can be safely described by a Fokker-Planck- and Langevin-type equation, when the number of intrinsic degrees of freedom is relatively large. In this case, the intrinsic subsystem exhibits a very interesting role as a finite heat bath and the fluctuation has the finite correlation time (as colored noise). Only when the number of degrees of freedom for the intrinsic system is infinite, the intrinsic subsystem can be treated as a statistical heat bath with white noise.

This study provides a general framework for studying the dissipative collective motion of such the systems as atomic, nuclei and biomolecule whose environment is not infinite.

\section{Acknowledgements}

The ideas developed in this chapter have matured through collaboration with my colleagues: Professors F. Sakata and Y. Zhuo. This work was partially supported by the National Natural Science Foundation of China under Grant Nos. 10475008 and 10975019, the Scientific Research Foundation for the Returned Overseas Chinese Scholars, Ministry of Personnel of China (Grant No.: MOP2006138) and the Japan-China cooperative research program organized by the Japan Society for the Promotion of Science.

\section{References}

[1] M. J. Klein, Science 169, (1970)361

[2] C. O. Escobar, L. F. dos Santos and P.C. Marques, Phys. Rev. A50 (1994) 1913.

[3] M. Gell-Mann and J. B. Hartle, Phys. Rev. D47,3345(1993).

[4] H. A. Kramers, Physica VII 4(1940)284.

[5] H. Rishen, The Fooker-Planck Equation, (Springer, Berlin, 1984).

[6] P. Hänggi, P. Talkner and M. Borkovec, Rev. Mod. Phys. Vol.62, 251(1990).

[7] P. Jung, J. G. Kissner, and P. Hänggi, Phys. Rev. Lett. 76, 3436(1996).

[8] I. Derányi, and R. D. Astumian, Phys. Rev. Lett. 82, 2623(1999).

[9] M. E. Fisher, and A. B. Kolomeisky, Physica A274, 241(1999).

[10] Y. Abe, S. Ayik, P.-G. Reinhard And E. Suraud, Phys. Rep. 275, 49(1996).

[11] H. A. Weidenmüller, Prog. Nucl. Part. Phys. 3, 49(1980).

[12] D. Hilscher, and H. Rossner,Ann. Phys. 17, 471(1992).

[13] S. Habib, K. Shizume and W. H. Zurek, Phys. Rev. Lett. 80, 4361(1998).

[14] Arjendu K. Pattanayak, Phys. Rev. Lett. 83, 4526(1999).

[15] J. Blocki et al., Ann. Phys. (N. Y.) 113, 330(1978).

[16] E. Ott, Phys. Rev. Lett. 42, 1628(1979). 
[17] M. Wilkinson, J. Phys. A23, 3603(1990);

M. Wilkinson and E. J. Austin, J. Phys. A28, 2277(1995)

[18] M. V. Berry and J. M. Robbins, Proc. R. Soc. London A442, 659(1993);

J. M. Robbins and M. V. Berry, J. Phys. A25, L961(1992).

[19] C. Jarzynski, Phys. Rev. A46, 7498(1992);

Phys. Rev.Lett. 74, 2937(1995); 71, 839(1993).

[20] S. E. Koonin, R. L. Hatch, and J. Randrup, Nucl. Phys. A283, 87(1977);

S. E. Koonin and J. Randrup, Nucl. Phys. A289, 475(1977).

[21] X. Wu,F. Sakata, Y. Zhuo and Z. Li, Phys. Rev. C48, 1183(1993)

[22] X. Wu,F. Sakata, Y. Zhuo, Z. Li and N. D. Dang, Phys. Rev. C53, 1233(1996).

[23] F. Sakata, M. Matsuo, T. Marumori and Y. Zhuo. Ann. Phys. (N.Y.) 194, 30(1989).

[24] G. Do Dang, A. Klein and P. -G Reinhard, Phys. Rev. C59, 2065(1996).

[25] D. Cohen, Phys. Rev. Lett. 78, 2878(1997); Phys. Rev. Lett. 82, 4951(1999).

[26] M. Bianucci, R. Mannella, B. J. West and P. Grigilini, Phys. Rev. E51, 3002(1995), and references therein.

[27] H. Hofmann, Phys. Rep. 284, 137(1997).

[28] M. Baldo, G. F. Burgio, A. Rapisarda and P. Schuck, Phys. Rev. C58, 2821(1998).

[29] H. C. Fogedby, Phys. Rev. E58, 1690(1998).

[30] S. Yan, F. Sakata, Y. Zhuo, and X. Wu, Phys. Rev. E63, 021116(2001).

[31] S. Yan, F. Sakata, and Y. Zhuo, Phys. Rev. E65, 031111(2002).

[32] F. Sakata, Y. Hashimoto, S. Yan, L. Guo, H. Imagawa, A. Seki, and S. Fujiwara, AIP Conf. Proc., Vol. 597, 249-257(2001)

[33] S. Yan, F. Sakata, and Y. Zhuo, Phys. Lett. A319, 60(2003).

[34] G. M. Zaslavsky, Physics of Chaos in Hamiltonian Systems: on the Foundations of Statistical Physics, (Imperial College Press, London, 1998).

[35] L. Boltzmann, Wiss. Ber. 58, 517(1968); 66, 275(1972)

[36] K. Huang, Statistical Mechanics, (Wiley,Singapore, 1987).

[37] K. Lindenberg and V. Seshadri, Physica A109,483(1981).

[38] B. Carmeli and A. Nitzan, Chem. Phys. Lett. 102,517(1983)

[39] O. Bohigas and M. G. Giannoni, Lecture notes in Phys. 209 (Springer, Heidelberg, 1984) 1, and references therein.

[40] S. Ayik and J. Randrup, Phys. Rev. C50, 2947(1994).

[41] S. Ayik, O. Yilmaz, A. Gokalp and P. Shuck, Phys. Rev. C58,1594(1998).

[42] G. F. Bertsch, P. F. Bortignon and R. A. Broglia, Rev. Mod. Phys., 55, 287(1983).

[43] J. da Providência, M. Yamamura and A. Kuriyama, Phys. Rev. C50, 1720(1994).

[44] A. Kuriyama, J. da Providência and M. Yamamura, Prog. Theor. Phys. 84, 1115(1990).

[45] A. Kuriyama, M. Yamamura, J. da Providência and C. da Providência, Phys. Rev. C45,2196(1992).

[46] M. Pettini and M. Landolfi, Phys. Rev. A41, 768(1990).

[47] M. Pettini and M. Cerruti-Sola, Phys. Rev. A44, 975(1991).

[48] L. Casetti and M. Pettini, Phys. Rev. E48, 4320(1993).

[49] E. Fermi, J. Pasta, and S. Ulam, in Collected Papers of Enrico Fermi (Accademia Nazionale dei Lincei and University of Chicago, Roma,1965), Vol. II, P.978.

[50] F. Sakata, T. Marumori, Y. Hashimoto and S. Yan, Suppl. Prog. Theor. Phys. 141, $1-111(2001)$

[51] J. R. Carry, Phys. Rep., 79, 129(1981).

[52] B. V. Chirikov, Phys. Rep., 52, 263(1979). 
[53] A. J. Lichtenberg and M. A. Lieberman, Regular and Stochastic Motion, Applied Mathematical Science 38 (Springer, New York, 1983).

[54] R. Kubo, Prog. Theor. Phys., 12,570(1957).

[55] R. Kubo, M. Toda, and N. Hashitsume, Statistical Physics II: Nonequilibrium Statistical Mechanics, Springer-Verlag, Now York 1985.

[56] R. Zwanzig, Ann. Rev. Phys. Chem. 16,67(1965).

[57] M. S. Green, J. Chem. Phys. 20,1281(1952).

[58] M. S. Green, J. Chem. Phys. 22,398(1954).

[59] R. Zwanzig, Ann. Rev. Phys. Chem. 16,67(1965).

[60] T. Marumori, T. Maskawa, F. Sakata, and A. Kuriyama, Prog. Theor. Phys. 64, 1294(1980).

[61] M. Bianucci, R. Mannella and P. Grigolini, Phys. Rev. Lett. 77, 1258(1996).

[62] E. Lutz and H. A. Weidenmüller, Physica A267, 354(1999).

[63] T. Marumori, Prog. Theor. Phys. 57, 112(1977).

[64] J. W. Negele, Rev. Mod Phys. 54, 913(1982).

[65] F. V. De Blasio, W. Cassing, M. Tohyama, P. F. Bortignon and R.A. Broglia, Phys. Rev. Lett. 68,1663(1992).

[66] A. Smerzi, A. Bonasera and M. Di Toro, Phys. Rev. C44,1713(1991).

[67] C. R. Willis and R. H. Picard, Phys. Rev. A9, 1343(1974).

[68] S. Nakajima, Prog. Theor. Phys.,20,948(1958)

[69] R. W. Zwanzig, J. Chem. Phys., 33,1338(1960).

[70] S. Y. Li, A. Klein, and R. M. Dreizler, J. Math. Phys. 11, 975(1970).

[71] A. N. Komologorov, Dokl. Akad. Nauk SSSR, 98, 527(1954).

[72] A. Bohr and B. R. Mottelson, Nuclear Structure I Benjamin, New York, 1969.

[73] W. H. Zurek and J. P. Paz, Phys. Rev. Lett. 72, 2508(1994);

W. H. Zurek, Phys. Rev. D24, 1516(1981);

E. Joos and H. D. Zeh, Z. Phys. B59, 229(1985).

[74] J. Ford, Phys. Rep. 5, 271(1992).

[75] H. Yoshida, Phys. Lett. A150, 262(1990).

[76] M. Sofroniou and W. Oevel, SIAM Journal of Numerical Analysis 34 (1997), pp. 2063-2086.

[77] N. G. van Kampen, Phys. Nor. 5, 279(1971)

[78] V. Latora and M. Baranger, Phys. Rev. Lett. 82, 520(1999).

[79] N. S. Krylov, Works on the Foundations of Statistical Physics, Princeton Series in Physics(Princeton University Press, Princeton, NJ, 1979).

[80] P. Grigolini, M. G. Pala, L. Palatella and R. Roncaglia, Phys. Rev. E 62, 3429(2000).

[81] U. M. S. Costa, M. L. Lyra, A. R. Plastino and C. Tsallis, Phys. Rev. E 56, 245(1997).

[82] V. Latora, M. Baranger, A. Rapisarda and C. Tsallis, Phys. Lett. A273, 97(2000).

[83] C. Tsallis, Fractals 3, 541(1995).

[84] C. Tsallis, J. Stat. Phys. 52, 479(1988);

E. M. E. Curado and C. Tsallis, J. Phys. A24, L69(1991); 24, 3187E(1991); 25, 1019E(1992);

C. Tsallis,Phys. Lett. A206, 389(1995).

[85] S. Yan, F. Sakata, Y. Zhuo and X. Wu, RIKEN Review, No.23, 153(1999).

[86] F. Sakata, T. Marumori, Y. Hashimoto and T. Une, Prog. Theor. Phys. 70, 424(1983). 


\section{Appendix}

Derivation of Eq. (104)

In this appendix, a derivation of the master equation (104) is discussed. From the definition in Eq. (103), one can get that the mean-field propagator $G_{\eta}\left(t, t^{\prime}\right)$ satisfies the relation

$$
\frac{d G_{\eta}\left(t, t^{\prime}\right)}{d t}=-i \lambda\left(\mathcal{L}_{\eta}+\mathcal{L}_{\eta}(t)\right) G_{\eta}\left(t, t^{\prime}\right)
$$

and has the properties

$$
\begin{aligned}
& G_{\eta}\left(t, t_{1}\right) G_{\eta}\left(t_{1}, t^{\prime}\right)=G_{\eta}\left(t, t^{\prime}\right) \\
& G_{\eta}^{-1}\left(t, t^{\prime}\right)=G_{\eta}\left(t^{\prime}, t\right)
\end{aligned}
$$

where $G_{\eta}^{-1}\left(t, t^{\prime}\right)$ is the inverse propagator of $G_{\eta}\left(t, t^{\prime}\right)$

With the aid of the mean-field propagator, the solution of Eq. (101) can be formally expressed as:

$$
\rho_{\eta}(t)=G_{\eta}(t, 0) \rho_{\eta}^{\prime}(t)
$$

which satisfies the equation

$$
\dot{\rho}_{\eta}(t)=\dot{G}_{\eta}(t, 0) \rho_{\eta}^{\prime}(t)+G_{\eta}(t, 0) \dot{\rho}_{\eta}^{\prime}(t)
$$

With Eq. (130), one gets

$$
\dot{\rho}_{\eta}(t)=-i \lambda\left(\mathcal{L}_{\eta}+\mathcal{L}_{\eta}(t)\right) G_{\eta}(t, 0) \rho_{\eta}^{\prime}(t)+G_{\eta}(t, 0) \dot{\rho}_{\eta}^{\prime}(t)
$$

Inserting Eq. (132) into the r.h.s. of Eq. (101) and comparing with Eq. (134), one can easily get

$$
\dot{\rho}_{\eta}^{\prime}(t)=-i \lambda \mathcal{L}_{\Delta, \eta}^{\prime}(t) \rho_{\eta}^{\prime}(t),
$$

where

$$
\mathcal{L}_{\Delta, \eta}^{\prime}(t)=G_{\eta}^{-1}(t, 0) \mathcal{L}_{\Delta, \eta}(t) G_{\eta}(t, 0)
$$

Eq. (135) is a linear stochastic differential equation. Applying cumulant expansion method(77), one has

$$
\begin{aligned}
\dot{\rho}_{\eta}^{\prime}(t) & =-i \lambda\left\langle\left\langle\mathcal{L}_{\Delta, \eta}^{\prime}(t)\right\rangle\right\rangle \rho_{\eta}^{\prime}(t) \\
& -\lambda^{2} \int_{0}^{t} d \tau\left\langle\left\langle\left\langle\mathcal{L}_{\Delta, \eta}^{\prime}(t) \mathcal{L}_{\Delta, \eta}^{\prime}(\tau)\right\rangle\right\rangle \rho_{\eta}^{\prime}(t)\right.
\end{aligned}
$$

where a symbol $\ll \cdots \gg$ denotes a cumulant defined as:

$$
\ll \mathcal{A B} \gg \equiv<\mathcal{A B}>-<\mathcal{A}><\mathcal{B}>
$$

which is related to the average over the intrinsic degrees of freedom

$$
<\cdots>\equiv \operatorname{Tr}\{\cdots\}
$$


Eq. (137) is valid upto the second order in $\lambda$. According to a definition of the fluctuation Hamiltonian $H_{\Delta, \eta}(t)$ in (100), the first-order term in (137) is zero since there holds a relation

$$
\left\langle\left\langle\mathcal{L}_{\Delta, \eta}^{\prime}(t)\right\rangle\right\rangle \sim\left\langle\left\langle\phi^{\prime}(t)\right\rangle\right\rangle=\left\langle\phi^{\prime}(t)\right\rangle=0
$$

one thus obtains

$$
\dot{\rho}_{\eta}^{\prime}(t)=-\lambda^{2} \int_{0}^{t} d \tau\left\langle\left\langle\mathcal{L}_{\Delta, \eta}^{\prime}(t) \mathcal{L}_{\Delta, \eta}^{\prime}(\tau)\right\rangle\right\rangle \rho_{\eta}^{\prime}(t)
$$

Inserting (140) into (134), one has

$$
\begin{aligned}
& \dot{\rho}_{\eta}^{\prime}(t)=-i \lambda\left(\mathcal{L}_{\eta}+\mathcal{L}_{\eta}(t)\right) G_{\eta}(t, 0) \rho_{\eta}^{\prime}(t) \\
& -\lambda^{2} \int_{0}^{t} d \tau G_{\eta}(t, 0)\left\langle\left\langle\mathcal{L}_{\Delta, \eta}^{\prime}(t) \mathcal{L}_{\Delta, \eta}^{\prime}(\tau)\right\rangle\right\rangle \rho_{\eta}^{\prime}(t)
\end{aligned}
$$

With the relation (131), (132) and (136), Eq. (141) can be read as

$$
\begin{aligned}
\dot{\rho}_{\eta}^{\prime}(t) & =-i \lambda\left(\mathcal{L}_{\eta}+\mathcal{L}_{\eta}(t)\right) \rho_{\eta}(t) \\
& -\lambda^{2} \int_{0}^{t} d \tau\left\langle\left\langle\mathcal{L}_{\Delta, \eta}(t) G_{\eta}(t, \tau) \mathcal{L}_{\Delta, \eta}(\tau)\right\rangle\right\rangle G_{\eta}(\tau, t) \rho_{\eta}(t)
\end{aligned}
$$

Making the variable transformation $\tau \longrightarrow t-\tau$, one can have

$$
\begin{aligned}
\dot{\rho}_{\eta}^{\prime}(t) & =-i \lambda\left(\mathcal{L}_{\eta}+\mathcal{L}_{\eta}(t)\right) \rho_{\eta}(t) \\
& -\lambda^{2} \int_{0}^{t} d \tau\left\langle\left\langle\mathcal{L}_{\Delta, \eta}(t) G_{\eta}(t, t-\tau) \mathcal{L}_{\Delta, \eta}(t-\tau)\right\rangle\right\rangle G_{\eta}(t-\tau, t) \rho_{\eta}(t)
\end{aligned}
$$

This is just Eq. (104). 


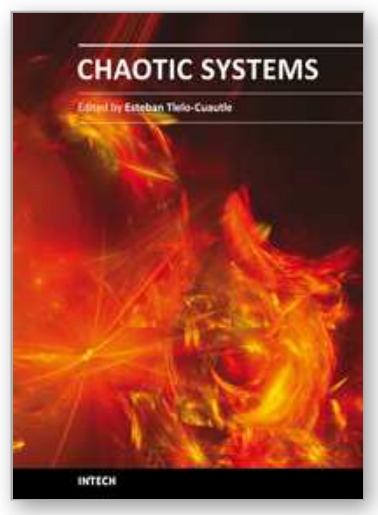

\author{
Chaotic Systems \\ Edited by Prof. Esteban Tlelo-Cuautle
}

ISBN 978-953-307-564-8

Hard cover, 310 pages

Publisher InTech

Published online 14, February, 2011

Published in print edition February, 2011

This book presents a collection of major developments in chaos systems covering aspects on chaotic behavioral modeling and simulation, control and synchronization of chaos systems, and applications like secure communications. It is a good source to acquire recent knowledge and ideas for future research on chaos systems and to develop experiments applied to real life problems. That way, this book is very interesting for students, academia and industry since the collected chapters provide a rich cocktail while balancing theory and applications.

\title{
How to reference
}

In order to correctly reference this scholarly work, feel free to copy and paste the following:

Shiwei Yan (2011). Microscopic Theory of Transport Phenomenon in Hamiltonian Chaotic Systems, Chaotic Systems, Prof. Esteban Tlelo-Cuautle (Ed.), ISBN: 978-953-307-564-8, InTech, Available from:

http://www.intechopen.com/books/chaotic-systems/microscopic-theory-of-transport-phenomenon-inhamiltonian-chaotic-systems

\section{INTECH}

open science | open minds

\section{InTech Europe}

University Campus STeP Ri

Slavka Krautzeka 83/A

51000 Rijeka, Croatia

Phone: +385 (51) 770447

Fax: +385 (51) 686166

www.intechopen.com

\section{InTech China}

Unit 405, Office Block, Hotel Equatorial Shanghai

No.65, Yan An Road (West), Shanghai, 200040, China

中国上海市延安西路65号上海国际贵都大饭店办公楼 405 单元

Phone: +86-21-62489820

Fax: +86-21-62489821 
(C) 2011 The Author(s). Licensee IntechOpen. This chapter is distributed under the terms of the Creative Commons Attribution-NonCommercialShareAlike-3.0 License, which permits use, distribution and reproduction for non-commercial purposes, provided the original is properly cited and derivative works building on this content are distributed under the same license. 